\title{
Optimizing the Tissue Engineering Of TUbULAR \\ Organ Structures by Bio-Printing
}

A Thesis Presented to

the Faculty of the Graduate School

at the University of Missouri-Columbia

In Partial Fulfillment

Of the Requirements for the Degree

Doctor of Philosophy

by

Ashkan Shafiee

Dr. Gabor Forgacs, Thesis Supervisor

December 2014 
The undersigned, appointed by the Dean of Graduate School, have examined the thesis entitled:

\author{
Optimizing the Tissue Engineering of \\ Tubular Organ Structures by Bio-Printing
}

presented by Ashkan Shafiee,

a candidate for the degree of Doctor of Philosophy and herby certify that, in their opinion, it is worthy of acceptance.

Dr. Gabor Forgacs

Dr. Ioan Kosztin

Dr. Gerald Meininger

Dr. Kevin Gillis

Dr. Gavin King 
دفتر دانش ما جمله بشوييد بـ مسى 


\section{ACKNOWLEDGMENTS}

First of all, I would like to truly and deeply thank my advisor, Dr. Gabor Forgacs, who is the most effective non-family member person through my entire life so far. His motivating support even when I was a master student in 2008, his invitation to join his group as a visiting scholar in 2009 and accepting me as a PhD student in 2010, as well as his advices over the last four years made him an unforgettable person in my life. Despite his busy schedule, he sincerely and patiently spent time with me and shared some of his knowledge that helped me to learn whatever I needed to successfully carry out my project.

I also would like to thank Dr. Ioan Kosztin for all of the fruitful discussions, time and help he kindly offered me through my PhD study. While I consider him as one of the best teachers I have ever had, by attending his class entitled "Computational Biological Physics", collaborating with Dr. Kosztin in my PhD project taught me a lot and I really enjoyed my research time with Dr. Kosztin and his group.

I consider myself fortunate to have had the chance to be part of the Physics Department of the University of Missouri, with its faculty and staff. I am especially indebted to my colleagues Dr. Jakab, Dr. Marga, Mr. Owens, Mr. McCune, Mr. Kremenak, Mr. Valizadeh, Mrs. Karimi and Dr. Sherafati for all the kind helps they extended me. And very special thanks to Dr. Bahram Mashhoon for everything I learned from him.

I would like to thank my PhD committee members; Dr. Gerald Meininger, Dr. Kevin Gillis and Dr. Gavin king. Their fruitful ideas and comments helped me a lot to learn more and finish this thesis more successfully.

I acknowledge the supporting funds throughout my PhD study. The work reported in this Thesis was supported by grants from the National Science Foundation PHY-0957914 and Frontier in Biological Research FIBR-0526854."

Lastly, I am exceptionally grateful to my family: my father who always made my goals seem so simple to achieve by giving me his supportive advice and my mother who gave me love to do what I wanted to do. I also would like to sincerely thank my wife, for all the motivation, support and happiness she brought into my life. I also thank my brother and sister, for their continuous support over the years. 
TABLE OF CONTENTS

ACKNOWLEDGMENTS ii

LIST OF ABBREVIATIONS vii

LIST OF TABLES IX

LIST OF FIGURES $\quad x$

LIST OF PARAMETERS $\quad X V$

ABSTRACT Xvi

CHAPTER I: INTRODUCTION

Tissue Engineering $\quad 2$

Tissue Engineering by self-assembly and bioprinting 6

$\begin{array}{ll}\text { Differential Adhesion } & 8\end{array}$

Tissue fusion $\quad 9$

Cell sorting 9

Cellular Particle Dynamics 10

CHAPTER II: MATERIALS AND METHODS 14

$\begin{array}{ll}\text { Human skin fibroblasts (HSFs) } & 14\end{array}$

Chinese Hamster Ovary (CHO) 15 
Fabrication of bioink particles 16

Effect of Cell concentration on aggregate size 18

CHAPTER III: MEASUREMENT OF TISSUE SURFACE TENSION 20

$\begin{array}{ll}\text { Surface Tensiometry } & 20\end{array}$

Parallel Plate Surface Tensiometry $\quad 21$

$\begin{array}{ll}\text { Theoretical determination of ATST } & 24\end{array}$

Experimental determination of ATST 26

Spheroids composed of human skin fibroblasts 28

Spheroids composed of Chinese Hamster Ovary cells 30

$\begin{array}{ll}\text { Conclusions } & 33\end{array}$

CHAPTER IV: FUSION OF SPHERICAL AGGREGATES 34

$\begin{array}{ll}\text { Theory } & 34\end{array}$

$\begin{array}{ll}\text { Experiments } & 39\end{array}$

Fusion of CHO spheroids: the initial linear regime 41

Fusion of CHO spheroids: the complete process 43

Fusion of fibroblast spheroids (same and different size) 44

Analysis of the fusion experiments: equal size aggregates $\quad 47$

Analysis of the fusion experiments: unequal size aggregates 49

Experimental calibration for CPD 56 
Fusion of spherical HSF aggregates $\quad 60$

Analysis of the fusion of spherical aggregates 61

Fusion of cylindrical HSF aggregates

Cylinder fusion in grooves $\quad 65$

Cylinder fusion on flat agarose surface $\quad 68$

$\begin{array}{ll}\text { Spheroid Fusion } & 69\end{array}$

$\begin{array}{ll}\text { Cylinder fusion } & 72\end{array}$

$\begin{array}{ll}\text { Conclusion } & 78\end{array}$

CHAPTER VI: EFFECT OF PREPARATION ON THE BIOMECHANICAL PROPERTIES OF BIOINK UNITS $\quad 80$

$\begin{array}{ll}\text { Apparent tissue surface tension (ATST) } & 80\end{array}$

$\begin{array}{ll}\text { Characteristic fusion time } & 81\end{array}$

$\begin{array}{ll}\text { Conclusions } & 86\end{array}$

CHAPTER VII: FINAL CONCLUSIONS AND FUTURE WORK 88

$\begin{array}{ll}\text { REFERENCES } & 91\end{array}$

Appendix A: Finding the ATST using "Exact Solution of Laplace equation" 97

Appendix B: Theory background for fusion of spherical aggregates 99 
VITA

102 


\section{LIST OF ABBREVIATIONS}

3D

ATST

ADSA

CPD

CPs

CC

CCD

$\mathrm{CHO}$

DAH

DMSO

DMEM

EHAM

EDTA

ECM

FBS

HSFs

IN
3-Dimensional

Apparent Tissue Surface Tension

Axisymmetric drop shape analysis

Cellular Particle Dynamics,

Cellular Particles

Central Core

Charge-coupled device

Chinese Hamster Ovary

Differential Adhesion Hypothesis,

Dimethyl Sulfoxide

Dulbecco's Modified Eagle Medium

Egg Holder aggregate Maker

Ethylenediaminetetraacetic acid

Extracellular Matrix

Fetal Bovine Serum

Human Skin Fibroblasts

Inner Chamber 


$\begin{array}{ll}\text { LA } & \text { Lower Assembly } \\ \text { LCP } & \text { Lower compression plate } \\ \text { OC } & \text { Outer Chamber } \\ \text { RPM } & \text { Revolution Per Minute } \\ \text { SM } & \text { Sausage Method } \\ \text { UCP } & \text { Upper Compression Plate }\end{array}$




\section{LIST OF TABLES}

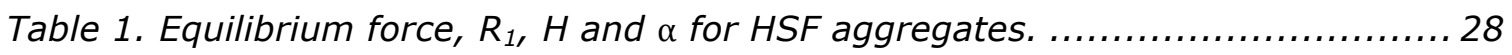

Table 2. Equilibrium force, $R_{1}, H$ and $\alpha$ for $\mathrm{CHO}$ aggregates.......................... 31

Table 3. Fusion results for 4 even HSF aggregate ............................... 54

Table 4. Fusion results for 3 uneven HSF aggregates ............................ 54

Table 5. Theoretical predictions for sample 1 in Table 4 using even samples in Table3. The average prediction is equal to 53.77 hours with $3 \%$ error with experimental results. .............................................................. 55

Table 6. Theoretical predictions for sample 2 in table 4 using even samples in Table3. The average prediction is equal to 54.34 hours with $12 \%$ error with experimental results. ......................................................................... 55

Table 7. Theoretical predictions for sample 3 in table 4 using even samples in Table3. The average prediction is equal to 51.87 hours with $10 \%$ error with experimental results. ......................................................................... 56

Table 8. Results for fusion of HSF aggregates prepared by sausage method ......... 71

Table 9. Comparison of data obtained with HSF aggregates prepared by EHAM and SM. While ATST is almost 50\% bigger for SM the viscosity is also $17 \%$ smaller than $(3.9 \times 108)$ that for EHAM. Hence one can state that larger ATST causes faster fusion. 84

Table 10. Effect of aggregate preparation on the viscoelastic properties of multicellular systems composed of HSF and $\mathrm{CHO}$ cells............................ 85 


\section{LIST OF FIGURES}

Figure 1. EHAM template....................................................... 16

Figure 2. Sausage method preparation: 1-after removing the cells from Petri dishes, they are transferred into a syringe. The syringe is capped using a robber stopper and then centrifuged. 2- through a hole in the syringe at the interface of the supernatant and cell pellet, the medium is removed. 3- The pellet inside the syringe is extruded into a glass capillary tube and incubated for $10-15$ minutes. ......................... 18

Figure 3. a. Aggregate prepared with $7.5 \mathrm{mill} / \mathrm{ml}$ cell concentration. b. Aggregate prepared with $15 \mathrm{mill} / \mathrm{ml}$ cell solution. 19

Figure 4. (Upper Panel) The surface tensiometry workstation. 1-monitor connected to side camera, 2-Cahn 2000, 3-Front camera, 4-light source, 5-Microbalance package. (Lower Panel) Schematics of the compressive force measurement. The outer chamber $(O C)$ is connected to a circulating water bath to keep the temperature of the inner chamber (IC) equal to the temperature of the water bath at $37^{\circ} \mathrm{C}$. The lower assembly (LA) screws into the base of the inner chamber. The IC has a central core (CC), whose tip is the lower compression plate (LCP). By means of a screw in the LA the distance between the two plates and thus the position of CC can be changed. During the compression the upper compression plate (UCP) is fixed and hangs from the arm of a recording electromagnetic microbalance. ................... 23

Figure 5. The Cahn MicroBalance, which operates on the null balance principle. By moving LCP upwards, the aggregate is squeezed between the compression plates, and a force is applied to UCP. This force is compensated by a torque motor with the same force and opposite direction. Hence the position of the UCP remains constant and the magnitude of the compensating force is recorded by the Cahn Microbalance.

Figure 6. The relevant geometric parameters. 26 
Figure 7. Recorded force vs. time for HSF aggregate. Peak force at the start of compression. Afterwards the aggregate is let to relax to equilibrium. Once the equilibrium is reached the aggregate is decompressed. Force is recorded during the entire relaxation process, in particular at equilibrium. 27

Figure 8. Upper panel: $\mathrm{F} / 2 \pi \mathrm{R}_{1}$ vs. $\alpha-1$ and the linear fit to obtain the slope and consequently ATST of HSF aggregates. Lower panel: compressed and uncompressed HSF aggregates. 30

Figure 9. Upper panel: $\mathrm{F} / 2 \pi \mathrm{R}_{1}$ vs. $\alpha-1$ and the linear fit to obtain the slope and consequently ATST of CHO aggregates. Lower panel: compressed and uncompressed $\mathrm{CHO}$ aggregates. 32 Figure 10. $R, r$ and the fusion angle for two identical fusing spherical aggregates... 35 Figure 11. Radii and fusion angles for the fusion of two different size aggregates... 38 Figure 12. The fusion workstation, an Olympus IX70 inverted microscope (1) attached to Cannon digital camera (2) attached to a monitor (3) through which fusing aggregates can be observed.......................................... 40 Figure 13. Fusion of equal size $\mathrm{CHO}$ aggregates (numbers denote time in hours). Special care was taken to record the shape of the fusing spheroids. In order to overcome the possible shaking of the construct (and thus making the recorded images inconsistent), the microscope was placed very close to the incubator, where the aggregates were kept between the recordings. This way the time needed to carry the Petri dish containing the aggregates from the incubator to the microscope was

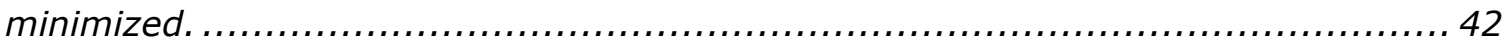
Figure 14. Variation of fusion time for $\mathrm{CHO}$ aggregates prepared with the EHAM method as obtained from the linear fit to the initial linear regime of fusion........... 43 Figure 15. Distribution of the characteristic fusion times for $8 \mathrm{CHO}$ samples prepared by EHAM as obtained from the exponential fit to the fusion experiments recorded for a week. 44 
Figure 16. Time $=0$ (placing aggregates close to each other) for 12 pairs of equal size

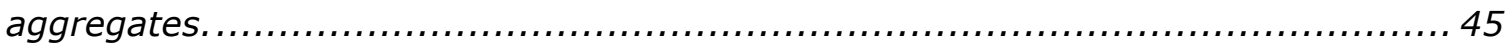
Figure 17. Time $=0$ (placing aggregates close to each other) for 10 pairs of unequal size aggregates. 46

Figure 18. Recording of shape evolution in a typical fusion experiment with unequal size aggregates, as followed for several days (numbers denote time in hours)...... 46 Figure 19. Rocording of shape evolution in a typical fusion of two equal sized HSF aggregates of $R_{0}=274$ micron. The solid-line contours represent the theoretical shapes of the fusing aggregates.

Figure 20. Theoretical fit (solid curve) to the experimental data (open circles) for $\sin ^{2} \theta$ vs. $\Delta \mathrm{t} / \tau$ for HSF aggregates of equal size corresponding to Figure 19. The dashed (dashed-dotted) curve represents the volume conserving theoretical result, with the same (effective) characteristic fusion time. Inset: time dependence of $R(t) / R_{0}$. The broken upper curve shows the variation of the ratio for the case of conserved volume. The strong deviation from the volume conserving upper curve, as shown in the lower curve (open circles: experimental results; solid curve: theoretical result using $R_{0}(t)=a(t) R_{0}(0)$ with $a(t)$ given in Eq. 4.3) implies a substantial decrease in volume during fusion. 49

Figure 21. Recording of shape evolution in a typical fusion experiment of two unequal HSF aggregates. 50 Figure 22. Theoretical fit (solid curve) to the experimental data (open circles) for $\sin ^{2} \theta$ vs. $\Delta t / \tau$ for HSF aggregates of unequal size, corresponding to Figure 21 . The dashed (dashed-dotted) curve represents the volume conserving theoretical result, with the same (effective) characteristic fusion time. 51 Figure 23. Left. Two similar size spherical aggregates prior to the initiation of fusion. Right. 24 well dish, each well covered with agarose containing pairs of similar size or different size aggregates in close proximity for fusion experiments. 61 
Figure 24. Upper Left. Pairs of fusing cylindrical aggregates in grooves. Upper Right. Pairs of fusing cylindrical aggregates in 6-well culture dish. Lower panel. Three pairs of fused cylinders embedded in agarose. ....................................... 63 Figure 25. Sample preparation for imaging. Cylindrical aggregates were cut in small pieces and were flipped to allow for the cross sectional images under the microscope. 64

Figure 26. Left. Cylindrical aggregates (white) inside agarose grooves. Right. Two fusing cylinders at $t=0$ under microscope. ...................................... 66

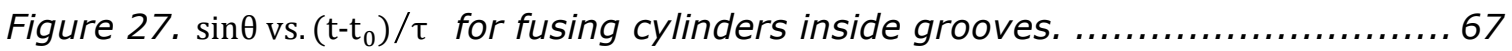
Figure 28. A fusion sequence for spherical HSF aggregates prepared by the sausage

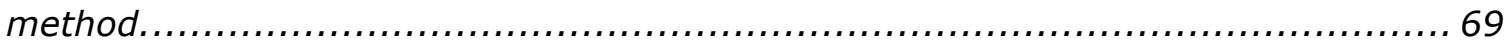
Figure 29. $\sin ^{2} \theta$ vs. $\left(\mathrm{t}-\mathrm{t}_{0}\right) \tau$ (upper panel) and volume decrease (lower panel) for fusing

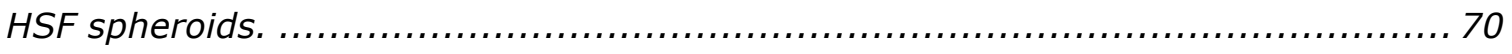

Figure 30. Cross sectional images of fusing cylinders at selected time points........ 73

Figure 31. $\sin \theta$ vs. $\left(\mathrm{t}-\mathrm{t}_{0}\right) / \tau$ for cylindrical HSF aggregates prepared with the sausage method. Red circles represent the average of $\sin \theta$ over 8 samples at each time point (except for the first data point for which see text). The solid line is the theoretical fit to the data. 74 Figure $32 . \sin \theta$ vs. $\left(\mathrm{t}-\mathrm{t}_{0}\right) / \tau$ for cylindrical HSF aggregates prepared with the sausage method. Open circles represent the average of $\sin (\Theta)$ over 8 samples at each time

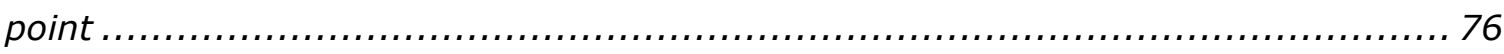
Figure 33. Length contraction of fusing cylinders. Open circles represent data averaged over 4 pairs of cylinders with the corresponding error bars. The solid line is the fit to Eq. (5.1) with $b_{0}$ and $\lambda_{b}$ equal to 0.28 and 0.05 respectively. 77 
Figure 34. Comparison of the characteristic fusion time for $\mathrm{CHO}$ aggregates prepared by different methods. The red squares and the blue diamonds represent fusion times obtained respectively for aggregates prepared by the EHAM and sausage method. . 82 


\section{LIST OF PARAMETERS}

Surface tension

Equatorial radius

$R_{1}$

Meridional radius

$R_{2}$

Polar radius

$R_{3}$

Distance between plates

$H$

Equilibrium force

F

Viscosity

$\eta$

Shear modulus

G

Characteristic fusion time

$\tau$

Initial radius of spherical aggregate $\quad R_{0}$

Initial length of cylinders $\quad L_{0}$

Rate of relaxation in volume decrease $\lambda$

Initial radius of big aggregate $\quad R_{10}$

$\begin{array}{ll}\text { Initial radius of small aggregate } & R_{20}\end{array}$

Angle of fusion $\theta$

Radius of interfacial ring of fusing aggregates $r$ 


\section{ABSTRACT}

Tissue engineering and regenerative medicine may help to save people's lives by fabricating new organs. Towards this goal our objective is to optimize the conditions for cells to self assemble into functional structures, such as tissues and eventually organoids. To facilitate self-assembly we employ the technology of bioprinting. To maintain the extended cellular assemblies, they need to be vascularized. Thus we first concentrated on the fabrication of blood vessels. We prepared convenient bioink particles, multicellular units composed of the relevant cell types and we deposited them into a configuration, consistent with the shape of the vessel. Self-assembly and the maturation of the construct takes place post-printing in special-purpose bioreactors by the fusion of the bioink units and the rearrangement of the cells within them. The time to achieve near physiological biomechanical properties has so far been found by trial and error. We report the experimental part of an experimental-theoretical-computational framework to optimize the postprinting maturation process, in particular the fusion of the bioink units. The connection between experiments and computer simulations were guided by theory. Here we report the results of extended fusion experiments and on their comparison with predictions of the theory. The excellent agreement we found, on one hand, provided a verification of the theoretical component of the formalism, and, on the other hand, the input for the computational component of the formalism. Specifically, our experiments, together with the theory, allowed the calibration of the basic simulation parameters, which in turn allows the full implementation of the computational component of the formalism to optimize the fabrication of blood vessels through the bioprinting process. 


\section{Chapter I: Introduction}

Tissue and organ failure due to injury or disease presents a major challenge for global health care (Persidis 1999). Often transplantation is the only remedy for the patient (Atala 2009). In 1954 Joseph Murray, Nobel laureate in Physiology or Medicine in 1990, performed the first successful organ transplantation when he implanted a healthy kidney recovered from Ronald Herrick into his identical twin brother Richard (Merrill et al. 1956; Murray et al. 2001; Starzl 1984). Since the procedure involved genetically identical siblings the danger of an adverse immune response was eliminated. In 1959 Murray went on to perform the world's first successful renal transplantation between nongenetically identical individuals (Murray et al. 1968).

Since Murray's pioneering operations the lives of many have been saved with organ transplantation. However the aging population, the lack of sufficient number of organ donors and the increasing number of people on the transplant waiting list necessitate the development of novel methods to restore the function of damaged organs and tissues (Atala 2012). One approach, based on tissue engineering, aims at mitigating the critical shortage of donor organs by the in-vitro fabrication of functional biological structures. 


\section{Tissue Engineering}

Tissue engineering combines the life sciences with the physical sciences and engineering to restore, replace or improve damaged tissues and organs (Langer \& Vacanti 1993; Lanza et al. 2013). In tissue engineering, biological constructs built from the patient's own cells, from another genetically non-identical individual or an animal are respectively referred to as autograft, allograft and xenograft (Lu et al. 2003). Besides implantation to improve organ function, engineered tissues are successfully being used in drug development and testing, potentially leading to substantial savings in the pharmaceutical industry.

More recently, tissue engineering, combined with developments in stem cell science, gene therapy, soluble molecules and reprograming of cell and tissue types is ushering in a new era in regenerative medicine (Vacanti 2010; Mason \& Dunnill 2008; Greenwood et al. 2006). Progress over the last 20 years has been spectacular. Functional tissues and organs have been fabricated by many teams around the globe helping to improve the quality of life of many patients (Sala et al. 2013).

In 2006 Atala and colleagues (Atala et al. 2006) reported the successful fabrication and implantation of autologous bladders for seven patients, aged 4-19 years, who had poorly compliant bladders 
(due to myelomeningocele). In their historical effort, Atala and colleagues obtained a bladder biopsy from each patient. They constructed a biodegradable bladder-shaped scaffold out of either collagen, or a composite of collagen and polyglycolic acid (that performed better in the long term found in their previous work (Yoo et al. 1998; Oberpenning et al. 1999)). The biopsied cells were cultured and seeded onto the scaffold. A few weeks after the biopsy the bladders were implanted into the patients. They then reported the successful follow-up for mean 46 months (Atala et al. 2006): they found that the engineered organs functioned normally.

As another spectacular accomplishment, in 2008, Macchiarini and colleagues reported the transplantation of a tissue-engineered airway for a 30-year-old woman who had suffered from tuberculous infiltration of the cervical trachea and left main bronchus (Macchiarini et al. 2008). They used a $7 \mathrm{~cm}$ tracheal segment from a cadaveric donor, which they used as a scaffold following its decellularization. Subsequently, they recellularized the matrix with the recipient's cells and employed it to replace the patient's damaged main left tracheal branch. The implantation was successful and the patient did not required immunosuppressive drugs (Macchiarini et al. 2008).

In 2011 and 2014 Atala and co-workers reported the tissue engineering of autologous urethras and vaginas (Raya-Rivera et al. 
2011; Raya-Rivera et al. 2014). In 2011 they reported on five boys, aged 10-14 years, with urethral defects, who received the transplanted organs. In their work Atala and co-workers cultured biopsied cells and seeded them on the tabularized polyglycolic acid: poly (lactide-coglycolide acid) scaffolds. The constructs were incubated for a week in Dulbecco's modified Eagle's medium. Generally, it took 4-7 weeks to form neo-urethras. After successful implantation the follow-up process was continued for a median 71 months (Raya-Rivera et al. 2011). In a similar experiment, in 2014 they reported on four girls, aged 13-18, who had been suffering from vaginal aplasia and who successfully received tissue-engineered autologous vaginas. They were followed-up for 3 years (Raya-Rivera et al. 2014).

The above impressive accomplishments involve scaffold-based tissue engineering and hollow spherical (e.g. bladder) and cylindrical (e.g. vagina and urethras) organs. The fabrication of solid functional organs such as liver, heart and kidney are still many years away.

There are three main approaches in tissue engineering, which respectively use only cells, cells and scaffold or just scaffold (Griffith \& Naughton 2002). Moreover, one may distinguish between scaffoldbased and scaffold-free tissue engineering approaches (Jakab et al. 2010). Scaffolds maybe made of natural (e.g. collagen, decellularized matrices) or synthetic materials and are supposed to mimic the natural 
3-dimensional environment, the extracellular matrix (ECM) for cells to grow and organize into tissues or organ structures with special shape (Lanza et al. 2013). Scaffolds need to be designed to be compatible with cell type(s) and the desired target tissue (Langer 2009). Thus, in principle, each engineered tissue or organ requires a different design and different materials. Furthermore the artificial scaffolds need to be fabricated with special properties, such as pore geometry, size, interconnectivity, and spatial distribution (Khademhosseini et al. 2006). The bulk and surface characteristics of scaffold materials may also affect cellular behaviors (Khademhosseini et al. 2006). Finally, the degradation of the scaffold must be compatible with the build-up of the ECM by the cells seeded in them.

The above requirements put severe constraints on the use of scaffolds. Therefore, despite recent progress in scaffold fabrication (Khademhosseini et al. 2009; Khademhosseini et al. 2006; Gauvin et al. 2012; Borenstein et al. 2002), scaffold-based tissue engineering faces numerous challenges, in particular in the case of solid organs. To overcome these challenges, it is perhaps not surprising, that recent efforts have been focused on combining tissue engineering with principles of developmental biology in general, and embryonic development in particular, to employ nature's abilities, rather than using scaffolds with their limitations. After all, nature designed the 
embryo as the ultimate tissue engineer. These efforts are converging towards the development of tissue and organ building methods that are scaffold-free (Marga et al. 2008).

\section{Tissue Engineering by self-assembly and bioprinting}

In order to smoothly integrate tissue engineering with biological and medical reality, practitioners of tissue engineering must incorporate in their work principles of cell biology (for example issues of cellular differentiation, growth and how ECM components affect cell function; (Langer 2007)), as well as developmental biology (for example how tissues and organs form and self-assemble in the developing embryo; (Ingber et al. 2006; Lenas et al. 2009; Basu \& Ludlow 2012)).

Embryonic development is a highly orchestrated sequence of events that is based on the complex interplay of genetic and molecular processes with biochemical and physical ones (Forgacs \& Newman 2005). As the embryo progresses from the initial, single cell zygote to the multicellular stage with many specialized differentiated cells and finally the multi-organ complex, it utilizes numerous morphogenetic self-assembly principles. The understanding of these processes is a prerequisite for their application in self-assembly based tissue engineering, the type to be used here (Jakab et al. 2010; Forgacs \& Newman 2005). 
The work reported in this thesis is motivated by scaffold free tissue engineering. In this approach only cellular material is used. Cells are provided with initial conditions consistent with the desired tissue or organ and let to self-assemble into the final product (in the course of which, among others, they secrete their own natural scaffold, the native ECM). In particular the initial conditions imply the delivery of cells into an initial configuration, compatible with the shape of the intended biological structure. For the delivery of cells into the initial configuration the Forgacs laboratory has developed a particular form of bioprinting (Jakab et al. 2004; Jakab et al. 2008; Norotte et al. 2009). In this approach "bioink" particles, multicellular aggregates of definite composition are delivered into a supporting environment, the "biopaper", according to computer-generated templates, consistent with the topology of the desired biological structure. Organoids form in the course of postprinting maturation by the fusion of the bioink particles and the sorting of cells within the bioink particles, both fundamental early developmental morphogenetic processes (see below). The advantage of this technology is that the bioink particles represent small three-dimensional tissue fragments. Thus cells in them are in a more physiologically relevant arrangement, in adhesive contact with their neighbors, which may assure the transmission of vital molecular signals. 


\section{Differential Adhesion}

One of the most important events that develops in the single cell zygote as it forms the multicellular organism is cell adhesion. In 1963, Steinberg formulated the "differential adhesion hypothesis" (DAH) and postulated that different cell types show different strengths of adhesion to each other as their specific inherent property (Steinberg 1963). Furthermore he stated that there is an analogy between multicellular systems in contact and immiscible liquids (e.g. oil and water). This, among others, implies that an initial random mixture of two different cell types is driven to reach a "phase-separated" final configuration by the minimization of interfacial and surface free energies that can be characterized by apparent tissue surface tensions (ATST). Apparent here implies that ATST is not identical to fluid surface tension based on physical properties, but rather a convenient effective parameter. In particular, the concept of ATST allows for the prediction of the shape and composition of the phase-separated multicellular assembly (i.e. in a cell spheroid which one of the two cell types surrounds the other). ATST has been widely used to interpret a number of early embryonic developmental processes (Lecuit \& Lenne 2007) and to analyze the in-vitro invasion pattern of tumors (Winters et al., 2005; Hegedüs et al., 2006). It also plays important role in bioprinting hence it will be studied comprehensively in Chapter III. 
DAH and its predictions have been observed and verified in numerous experiments and ATST measured by many groups (Steinberg 1963; Steinberg 1978; Steinberg 1998; Armstrong 1989; Mombach et al. 1995; Foty et al. 1994; Foty et al. 1996; Duguay et al. 2003; Norotte et al. 2008; Mgharbel et al. 2009; Marmottant et al. 2009)

\section{Tissue fusion}

Tissue fusion is a self-assembly process in which two or more distinct multicellular assemblies (such as multicellular aggregates, tissue fragments, etc.) in contact, merge and coalesce. It is a fundamental morphogenetic process that gives rise to the formation of complex structures out of simpler ones in the course of early development or disease. Tissue fusion is analogous to the fusion of liquid droplets in accordance with Steinberg's notion of tissue liquidity (Pérez-Pomares \& Foty 2006). This self-assembly process will be heavily relied on in the bioprinting process (see Chapter IV and V).

\section{Cell sorting}

Cell sorting is another early morphogenetic phenomenon that gives rise to compartmentalization in the embryo and eventually the separation between tissues and organs (Pérez-Pomares \& Foty 2006). 
In particular, when two cell types are randomly mixed in an aggregate, cell sorting leads to the sorting of one cell type into the interior of the aggregate surrounded by the other cell type. DAH provides a simple molecular explanation for this phenomenon: it is the more adhesive cells that sort to the interior with this maximizing their mutual adhesion and minimizing the configurational energy of the assembly. As ATST correlates with the strength of adhesion, DAH predicts that the more adhesive cells should have a higher apparent tissue surface tension. This prediction has been confirmed in numerous in vitro experiments (Foty et al. 1996; Duguay et al. 2003; Foty \& Steinberg 2005; Damon et al. 2008).

\section{Cellular Particle Dynamics}

Most tissue engineering methods employed presently lack quantitative predictability and are mostly of trial and error type. When scaffolds are used, it is hoped that cells grow in the scaffold as if they were within their own native ECM. How the geometry of the scaffold affects cell functions is not quantitatively predicted. In the scaffold free bioprinting approach it is a priori not known how long postprinting maturation needs to continue before the structure can be implanted into a living organism. Motivated by the desire to optimize the bioprinting of tubular organ structures by the bioprinting method described above 
Kosztin and collaborators developed a predictive "theoretical, experimental and computational" framework, the Cellular Particle Dynamics (CPD; (Kosztin et al. 2012)). Here we briefly recapitulate the salient points of CPD.

CPD is a computer simulation framework to predict the time evolution of the biomechanical relaxation process of multicellular systems (Kosztin et al. 2012; McCune et al. 2014). In CPD, a cell is initially considered as a continuum material object with constant volume and flexible shape and coarse-grained into a finite number of equal volume elements. Point-like cellular particles (CPs) located at the elements' center of mass represent these elements. CPs within the same cell and in different cells interact via judiciously chosen attractive and repulsive interactions. CPs are kept together by a confining potential which plays the role of an effective cell membrane. The combination of attractive and repulsive interactions determines the effective size of a CP. CPs move according to over-damped Langevin dynamics and their motion determines the change of the cells' shape in time and thus the time evolution of the shape of the multicellular system, which is followed by recording the trajectories of all CPS through the integration of their equations of motion.

The CPD force fields are constructed in such a way that the simulated multicellular system behaves as a complex viscous liquid. In 
particular, the CPD parameters, for any cell type, are determined from "calibration experiments", for example the fusion of two spherical aggregates. CPD simulation and experiment are related by the theoretical analysis of the "calibration experiment". For example a simple continuum model relates the time scale of simulations to the time scale of experiments.

The objectives of this thesis work are: (i) provide the experimental basis for $\mathrm{CPD}$, in particular to calibrate the CPD parameters and (ii) to validate the predictions of CPD through a collaborative project with the Kosztin group. The theoretical and computational components of the CPD were studied by the Kosztin group, whereas all the experimental work, as described below, was carried out in the Forgacs laboratory.

The following four chapters describe the experimental work that comprises this thesis. In Chapter II the materials and methods are discussed. In particular it presents the "egg holder aggregate maker" EHAM and sausage method, two different ways to fabricate multicellular aggregates that serve as bioink particles. In Chapter III we describe the measurement of the apparent surface tension of those tissues that we use for the calibration of CPD. To validate CPD, fusion experiments for the spherical and cylindrical bioink particles are presented in Chapters IV and V. Fusion experiments with spherical 
aggregates, prepared with the EHAM method, were performed for pairs with equal diameter as well as differing diameters and results compared with the corresponding simulations using the theoretical component of the formalism. In Chapter $\mathrm{V}$ the fusion of spherical and cylindrical aggregates prepared with the sausage method are discussed and compared with the corresponding simulations. Chapter VI describes the effect of the method of aggregate preparation on properties of the multicellular systems. In Chapter VII we conclude with summarizing the accomplishments reached in this thesis and putting forward some thoughts for future work. 


\section{Chapter II: Materials and Methods}

In this thesis we have used two different cell types: Chines Hamster Ovary ( $\mathrm{CHO}$ ) and Human Skin Fibroblast (HSF). CHO cells are easy to grow and work with. In addition the previous results of viscoelastic studies on $\mathrm{CHO}$ cells by previous members of the Forgacs lab could be compared with the new results. Fibroblasts were used as they had been employed earlier for bioprinted vessels. Here we used and studied aggregates of fibroblasts in order to optimize the engineering of tubular organ structures such as blood vessels by bioprinting.

\section{Human skin fibroblasts (HSFs)}

HSF cells were purchased from the American Type Culture Collection (CRL-2522; ATCC, Manassas, VA). Cells were cultured in Dulbecco's Modified Eagle Medium (DMEM) with F12 Nutrient Mixture (Ham's), antibiotics (Penicillin/streptomycin and Gentamicin), 2mM glutamine, 0.1 M Sodium Pyruvate (all chemicals purchased from Life Technologies Gibco; Green Island, NY), 20\% Fetal Bovine Serum (FBS; Thermo Fisher, Waltham, Massachusetts) on $0.5 \%$ gelatin coated dishes (Sigma, St. Louis, MO) and were maintained at $37^{\circ} \mathrm{C}$ in a humidified atmosphere containing $5 \% \mathrm{CO}_{2}$. In each subculture, the cells were washed twice with PBS and detached using a $0.1 \%$ Trypsin 
EDTA (Ethylenediaminetetraacetic acid) solution. Trypsin was purchased from (Life Technologies Gibco; Green Island, NY) and EDTA was purchased from (Sigma, St. Louis, MO). For cylinder fixation described in Chapter $\mathrm{V}$ paraformaldehyde was purchased from Alfa Aesar (Ward Hill, MA).

\section{Chinese Hamster Ovary (CHO)}

$\mathrm{N}$-cadherin transfected $\mathrm{CHO}$ cells (courtesy of A. Bershadsky, Weizmann Institute, Rehovot, Israel) were grown at $37^{\circ} \mathrm{C}$ with $5 \% \mathrm{CO}_{2}$ in DMEM supplemented with $10 \%$ FBS, antibiotics $(100 \mathrm{U} / \mathrm{mL}$ penicillin streptomycin and $25 \mu \mathrm{g} / \mathrm{mL}$ gentamicin), $2 \mathrm{mM} \mathrm{L-glutamine,} 0.1 \mathrm{M}$ Sodium Pyruvate.

\section{Cryopreservation}

To assure that all cells had same the passage number, following cell culturing to reach sufficient number of cells aliquots of cells were cryopreserved. After trypsinization, cells were pelleted via $5 \mathrm{~min}$ centrifugation at $2000 \mathrm{rpm}$ and were re-suspended in a freezing medium consisting of $10 \%$ dimethyl sulfoxide (DMSO, Acros Organics, NJ) and 90\% FBS. The solution was transferred to cryogenic screw cap vials (Fischer Scientific, Pittsburg, PA) and frozen in freezing canisters (Nalgene, NY) containing room temperature isopropyl alcohol and incubated in a $-80^{\circ} \mathrm{C}$ freezer for 24 hours. Subsequently, cells were 
stored in a liquid nitrogen tank until use.

\section{Fabrication of bioink particles}

Multicellular aggregates or mini-tissues were fabricated using HSF and $\mathrm{CHO}$ cells in spherical and cylindrical shape using two different methods, the "egg holder aggregate maker" (EHAM) and sausage method. In the EHAM method small holes $1 \mathrm{~mm}$ in diameter, were created in an agarose mold using special plastic templates (Figure 1), and this assembly used as a multi-well plate. Cells were trypsinized and removed from Petri dishes and centrifuged in $15 \mathrm{ml}$ tubes for 5 min at $2000 \mathrm{rpm}$. Subsequently, cells were counted and remixed with medium with different concentrations. For the experiments in Chapter IV the cell solutions of 4,8 and 16 million cells per milliliter of medium have been used to have different size of spherical aggregates. The cell solutions were pipetted quickly over the holes and incubated for 48 hours. During this time cells settled down in the holes, and formed spherical cell aggregates due to the minimization of surface energy.
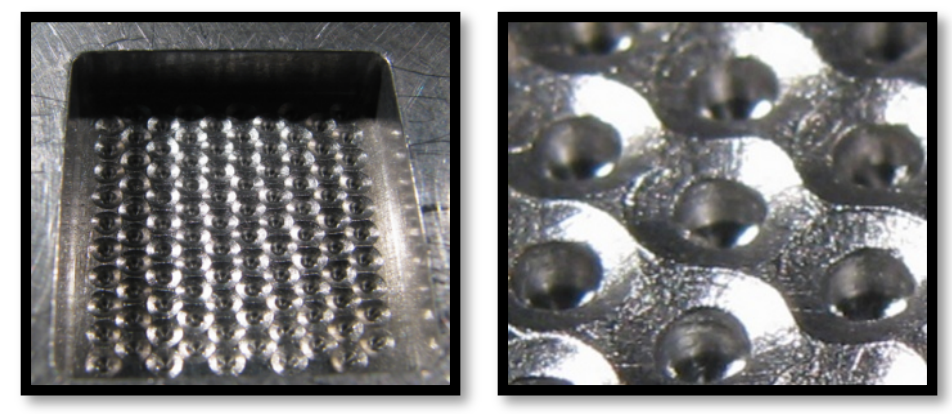

Figure 1. EHAM template. 
For some experiments the sausage method was used to prepare spherical and cylindrical aggregates. In this case cells were trypsinized and removed from the Petri dishes and were centrifuged in $15 \mathrm{ml}$ tubes for $5 \mathrm{~min}$ at $2000 \mathrm{rpm}$. Subsequently, the medium was discarded and the resulting pellet of cells was dissolved in $300 \mu \mathrm{l}$ of fresh medium using gentle pipetting action, and transferred to a $1 \mathrm{ml}$ syringe. The syringe was capped with a robber stopper and was centrifuged for 2 minutes at $2000 \mathrm{rpm}$. The supernatant forming in the syringe was siphoned off via a hole made by a needle in the syringe, at the cell pellet-supernatant interface. The remaining cell paste was then aspirated into a glass capillary of $500 \mu \mathrm{m}$ inner diameter and the cell paste containing capillary incubated for 15 minutes in medium. Next, the content of the capillary was extruded using a metal piston. The resulting "cell sausage" was used to prepare cylindrical cell aggregates of desired length. For spherical aggregates, cylinders were cut into pieces with aspect ratio 1 . These small cylinders were kept in 10-ml tissue culture flasks (Bellco Glass, Vineland, $\mathrm{NJ}$ ) with $2.5 \mathrm{ml}$ of medium on a gyratory shaker at $120 \mathrm{rpm}$ with $5 \% \mathrm{CO} 2$ at $37^{\circ} \mathrm{C}$, for 48 hours, during which time they spontaneously round up into spherical aggregates. Figure 2 shows the processes of preparing the multicellular past like sausages using a syringe. 


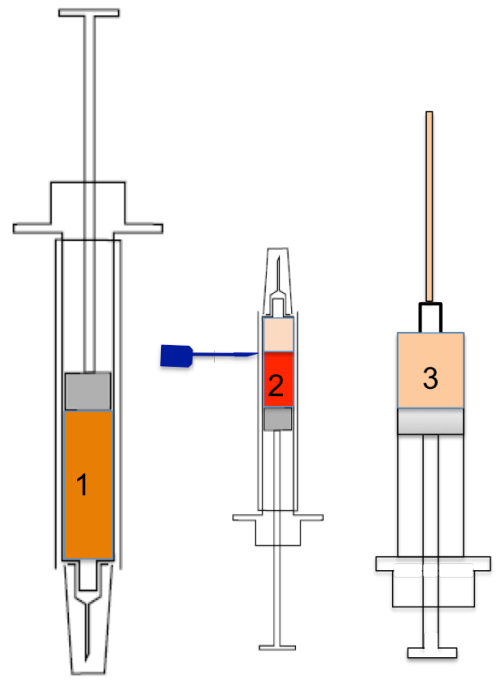

Figure 2. Sausage method preparation: 1-after removing the cells from Petri dishes, they are transferred into a syringe. The syringe is capped using a robber stopper and then centrifuged. 2- through a hole in the syringe at the interface of the supernatant and cell pellet, the medium is removed. 3- The pellet inside the syringe is extruded into a glass capillary tube and incubated for 10-15 minutes.

\section{Effect of Cell concentration on aggregate size}

To control the size of aggregates prepared by EHAM we studied the effect of cell concentration on aggregate size. For this we prepared cell solutions in three different concentrations, 7.5, 8.5 and 15 million cells per $\mathrm{ml}$ of medium. Figure 3 shows that cell concentration during aggregate preparation may affect aggregate size. 


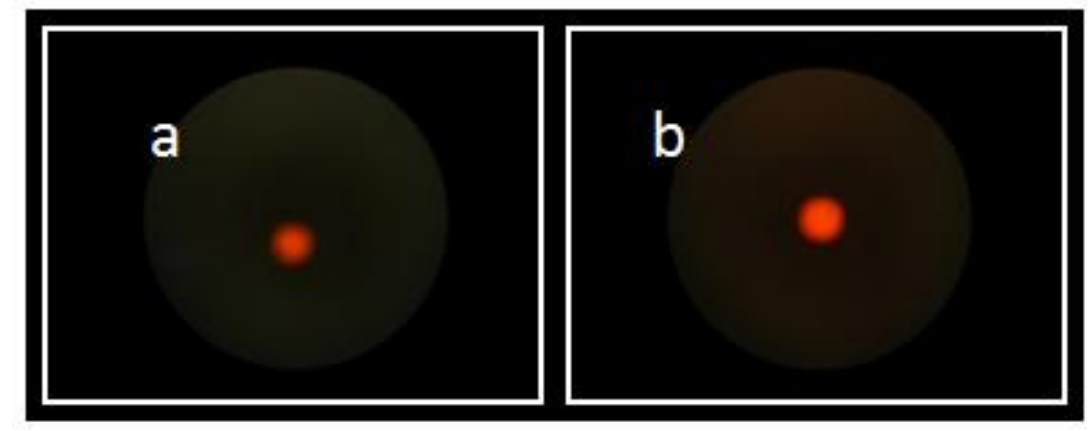

Figure 3. a. Aggregate prepared with $7.5 \mathrm{mill} / \mathrm{ml}$ cell concentration. b. Aggregate prepared with $15 \mathrm{mill} / \mathrm{ml}$ cell solution.

The fact that one can control the size of the cell aggregates just with changing the concentration of the cell solution helps to make different size aggregate. For this purpose in experiments described in Chapter IV cell solutions with concentration of 5,10 and 16 million cells $/ \mathrm{ml}$ were used. Considering that the number of wells in each EHAM template is 100 and the amount of cell solution used for a template is $0.3 \mathrm{ml}$, one can estimates that each aggregate consists of 15,000 , 30,000 and 48,000 cells respectively.

Further details on the experiments will be given in each chapter where they are described. 


\section{Chapter III: Measurement of Tissue Surface Tension}

In this chapter we describe measurements of Apparent Tissue Surface Tension (ATST, denoted by $\gamma$ ), which is needed for the calibration of CPD to predict the characteristic fusion time described in Chapters IV and V. ATST is the macroscopic manifestation of molecular adhesion between cells and provides a quantitative measure for the cohesion of the tissue (Foty et al. 1996).

\section{Surface Tensiometry}

Since it has been introduced by Steinberg, the importance of ATST has been recognized by many investigators and several methods to measure it have been devised. Kalantarian and coworkers (Kalantarian et al. 2009) used the pendant drop method and axisymmetric drop shape analysis (ADSA), as a novel numerical method for imperfect profiles to determine $\gamma$. Guevorkian and co-authors (Guevorkian et al. 2010) used the micropipette aspiration technique to test the mechanical properties of multicellular spheroids and to determine their surface tension, viscosity, and elastic modulus. They aspirated spherical aggregates into a pipette with a diameter considerably smaller than that of the aggregate. (This technique was previously 
used to find the viscoelastic properties of single cells (Sato et al. 1990; Evans \& Yeung 1989)).

The most common method, however, is the compression plate tensiometry (Foty et al. 1994; Foty et al. 1996; Foty \& Steinberg 2005; Hegedüs et al. 2006; Schötz et al. 2008; Mgharbel et al. 2009; Manning et al. 2010; Forgacs et al. 1998). In this method an aggregate is compressed between two parallel plates and the aggregate let to equilibrate under the compressive force. The equilibrium force, through the Laplace equation and certain geometrical parameters, is related to ATST. In 2008 Norotte and coauthors (Norotte et al. 2008) solved exactly the Laplace equation for the parallel plate geometry and provided an effective and reliable method to experimentally determine ATST.

\section{Parallel Plate Surface Tensiometry}

The surface tension of cellular spheroids composed of $\mathrm{CHO}$ and HSF cells were measured using an in-house built parallel plate surface tensiometer (Figure 4 up). In this method spherical aggregates are compressed between two parallel plates and one measures the force exerted on the upper plate as the aggregate relaxes to its compressed equilibrium. 
The lower panel of Figure 4 shows the schematic view and operation of the parallel plate chamber. The upper compression plate (UCP), which hangs from the arm of a recording Cahn/Ventron (Cerritos, CA) model 2000 electromagnetic microbalance (Figure 5), is fixed in its position, as the microbalance operates on the null balance principle. Thus, when a compressive force is applied to the UCP the microbalance generates a force of the same magnitude and opposite direction.

Once the aggregate is compressed, a force is applied to the upper plate, which produces a force about the axis of rotation in microbalace. The torque motor (Figure. 5) generates an electric current to produce a force about the same axis that is equal and opposite to the force acting on the upper plate, thus forcing the beam to remain in its original (null) position. The bigger the force on the upper plate the bigger the current produced by the torque motor, which is thus the direct measure of the force acting on the beam. 


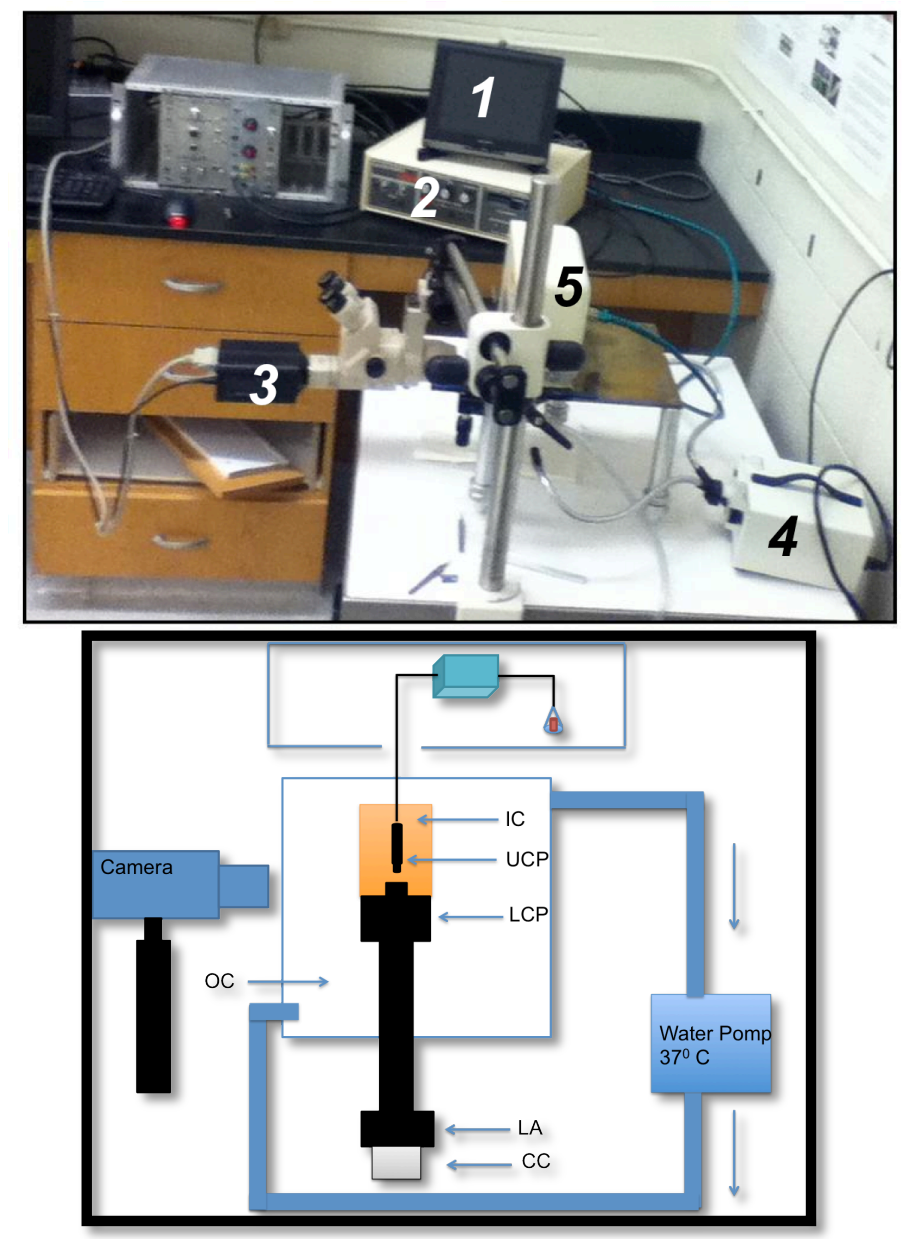

Figure 4. (Upper Panel) The surface tensiometry workstation. 1-monitor connected to side camera, 2-Cahn 2000, 3-Front camera, 4-light source, 5-Microbalance package. (Lower Panel) Schematics of the compressive force measurement. The outer chamber $(\mathrm{OC})$ is connected to a circulating water bath to keep the temperature of the inner chamber (IC) equal to the temperature of the water bath at $37^{\circ} \mathrm{C}$. The lower assembly (LA) screws into the base of the inner chamber. The IC has a central core (CC), whose tip is the lower compression plate (LCP). By means of a screw in the LA the distance between the two plates and thus the position of $\mathrm{CC}$ can be changed. During the compression the upper compression plate (UCP) is fixed and hangs from the arm of a recording electromagnetic microbalance. 


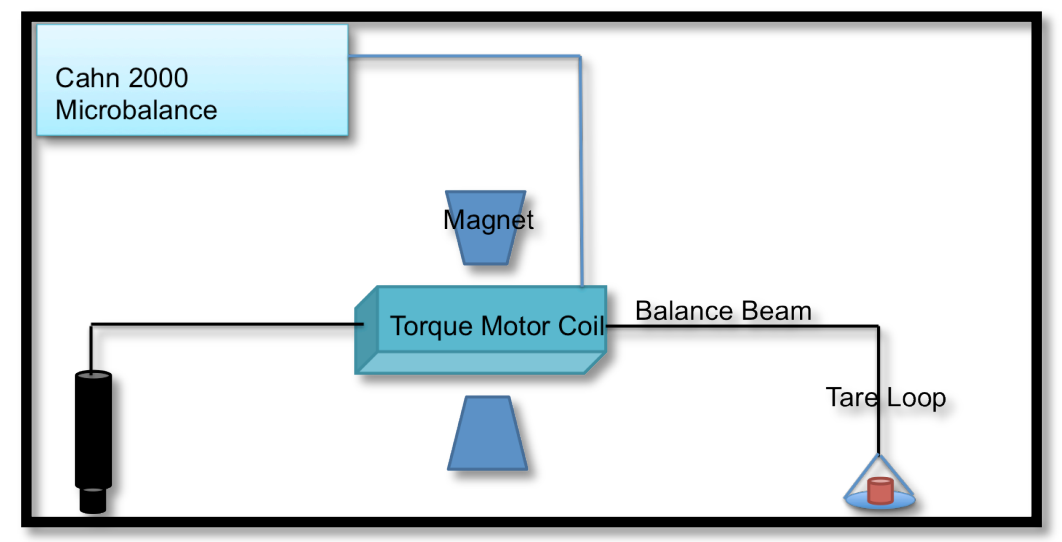

Figure 5. The Cahn MicroBalance, which operates on the null balance principle. By moving LCP upwards, the aggregate is squeezed between the compression plates, and a force is applied to UCP. This force is compensated by a torque motor with the same force and opposite direction. Hence the position of the UCP remains constant and the magnitude of the compensating force is recorded by the Cahn Microbalance.

This force of the microbalance is recorded using Labview (National Instruments, TX). The shape of the aggregate before and after the compression and after the decompression is recorded with a CCD camera (Diagnostic Instruments, MI) that is attached to a horizontally positioned dissecting microscope ( 3 in the lower panel of Figure 4; Olympus, Japan).

\section{Theoretical determination of ATST}

Figure 6 defines the important geometrical parameters that are needed to determine the aggregate's surface tension. These are related to the Laplace pressure exerted by the aggregate on the upper 
compression plate once the compressed aggregate reaches equilibrium. The Laplace pressure is determined from the equilibrium force (measured by the Cahn microbalance) acting on the aggregate. The surface tension is determined from the Laplace equation that relates the Laplace pressure to the geometric parameters (Norotte et al. 2008). The Laplace equation, in general, is a partial differential equation whose solution depends on boundary conditions. This equation has been used in various forms to deduce ATST. Early works used a form that related the equilibrium force and $R_{1}, R_{2}$ and $R_{3}$ to the surface tension (Foty et al. 1984, 1986). Later Norotte and co-workers provided the exact solution of the Laplace equation for the geometry of the compressed aggregate, which required only the more accurately measurable quantities $R_{1}$ and $H$ (Figure 6 ). In our work we have used the solution obtained by Norotte and co-workers (details are provided in Norotte et al. 2008). 


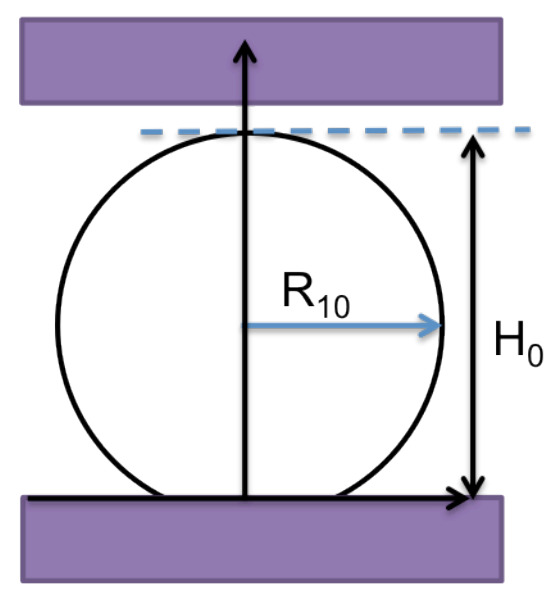

Before Compression

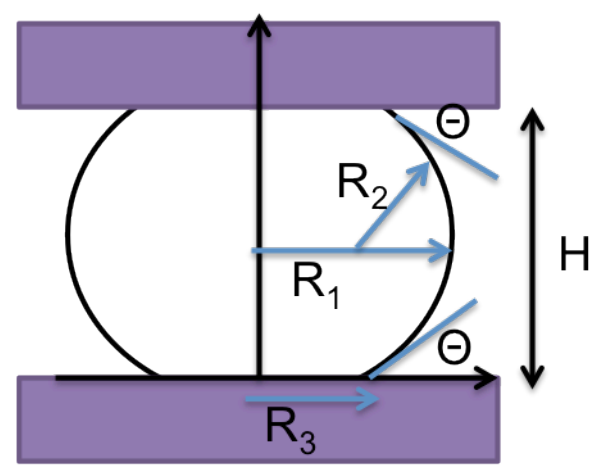

After Compression

Figure 6. The relevant geometric parameters.

\section{Experimental determination of ATST}

To determine the aggregate's surface tension one needs to measure the equilibrium compressive force and the geometric parameters (Figure 6). The equilibrium force is measured using the Cahn Microbalance. Figure 7 shows a typical force relaxation process for HSF aggregates (as recorded with Labview). The equilibrium force corresponds to the horizontal portion of the curve. (To find the force in Newtons, one needs to multiply the milligram values in the curve by the numerical value of the gravitational constant, $9.8 \times 10^{3}$. As shown in Figure 7 , equilibrium is reached typically in about 30 min postcompression (the initial peak value of the force)). 
If the liquid analogy holds for the studied aggregates then the measured ATST must be independent of the magnitude of the compressive force and the decompressed aggregate must return to its pre-compressed spherical shape. We verified that these conditions are indeed fulfilled.

In addition cell aggregates show elastic and viscous properties in short and long term under compression. Forgacs and co-authors (Forgacs et al. 1998) showed how to obtain the viscosity and shear modulus of the model tissue that composes the aggregate from the force relaxation diagram (Figure 7).

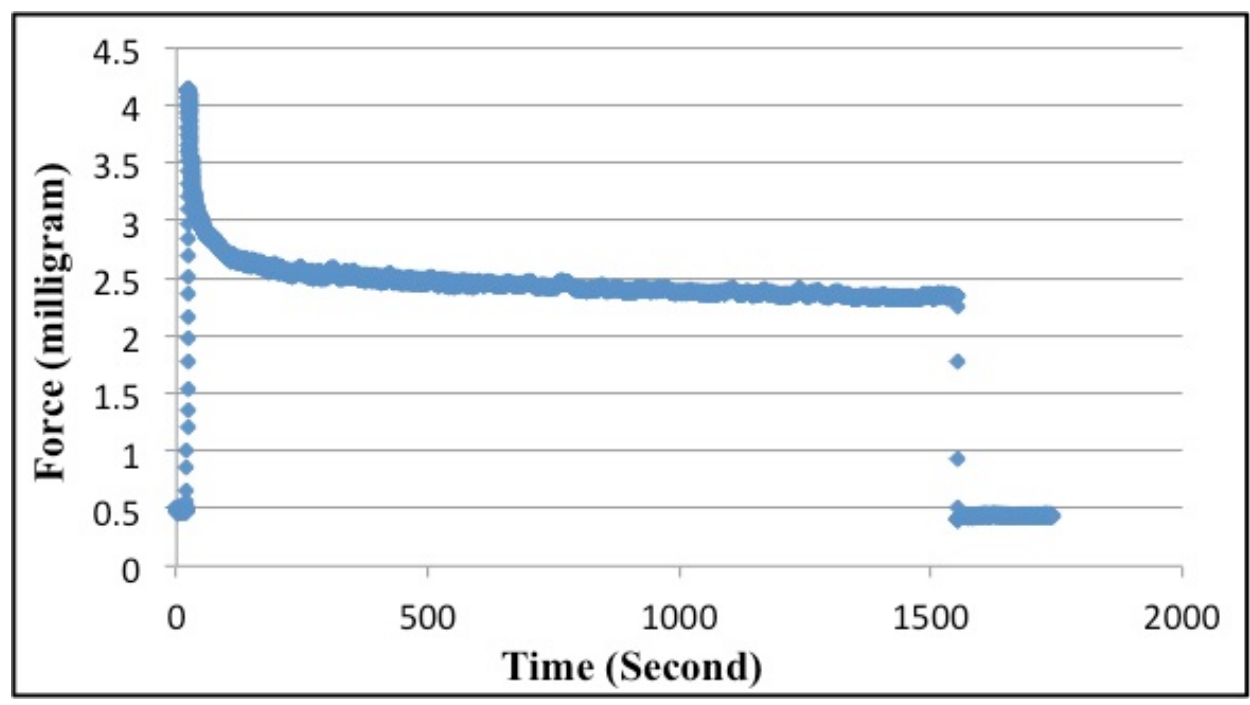

Figure 7. Recorded force vs. time for HSF aggregate. Peak force at the start of compression. Afterwards the aggregate is let to relax to equilibrium. Once the equilibrium is reached the aggregate is decompressed. Force is recorded during the entire relaxation process, in particular at equilibrium. 


\section{Spheroids composed of human skin fibroblasts}

The surface tension of HSF aggregates, prepared by the EHAM method was measured using the parallel plates apparatus and evaluated using the exact solution of the Laplace equation (Norotte et al. 2008). To use the Laplace equation we recorded the post-compressive equilibrium force and some geometrical parameters, such as $R_{1}$ and $H$, which are listed for 8 different HSF aggregates in Table 1.

\begin{tabular}{|c|c|c|c|c|c|}
\hline Sample & Force (dyne) & $\mathrm{R}_{1}(\mathrm{~mm})$ & $\mathrm{H}(\mathrm{mm})$ & $\mathrm{H} /\left(2 \mathrm{R}_{1}\right)$ & $\alpha$ \\
\hline 1 & 0.686 & 0.254 & 0.411 & 0.810 & 1.086 \\
\hline 2 & 2.022 & 0.257 & 0.382 & 0.744 & 1.134 \\
\hline 3 & 1.274 & 0.251 & 0.391 & 0.779 & 1.107 \\
\hline 4 & 2.547 & 0.255 & 0.349 & 0.685 & 1.185 \\
\hline 5 & 1.343 & 0.222 & 0.271 & 0.611 & 1.265 \\
\hline 6 & 3.577 & 0.231 & 0.214 & 0.462 & 1.515 \\
\hline 7 & 2.646 & 0.218 & 0.223 & 0.511 & 1.416 \\
\hline 8 & 2.450 & 0.218 & 0.232 & 0.534 & 1.376 \\
\hline
\end{tabular}

Table 1. Equilibrium force, $\mathrm{R}_{1}, \mathrm{H}$ and $\alpha$ for $\mathrm{HSF}$ aggregates. 
The surface tension, as provided by the solution of Norotte et al. is obtained from the equation $F / 2 \pi R_{1}=\gamma(\alpha-1)$. Here $F$ is the measured equilibrium force, $\gamma$ is the ATST and $\alpha$ is a quantity that depends on the combination of the geometric parameters and is obtained numerically through the solution of an integral equation (for details see Norotte et al. 2008).

Once $\alpha$ is determined, the surface tension can be found from the tangent of the graph of $\left(F / 2 \pi R_{1}\right)$ versus $\alpha-1$ as shown in Figure 8 , where the diamond symbols correspond to the 8 measurements listed in Table 1.

The slope of the graph in Figure 8 is 49.77 , which corresponds to an ATST of 49.77 dyne/cm for HSF aggregates prepared by the EHAM method. As we expected the surface tension was found to be independent of the size of the aggregates and the magnitude of the initial compressive force applied to the aggregates. Furthermore based on the force relaxation diagram one can obtain the model tissue's viscosity and shear modulus, respectively $\eta=1.7 \times 10^{6}\left(\right.$ dyne. $\left.\mathrm{sec} / \mathrm{cm}^{2}\right)$ and $G \approx 19 \times 10^{3}$ (dyne $/ \mathrm{cm}^{2}$ ). 


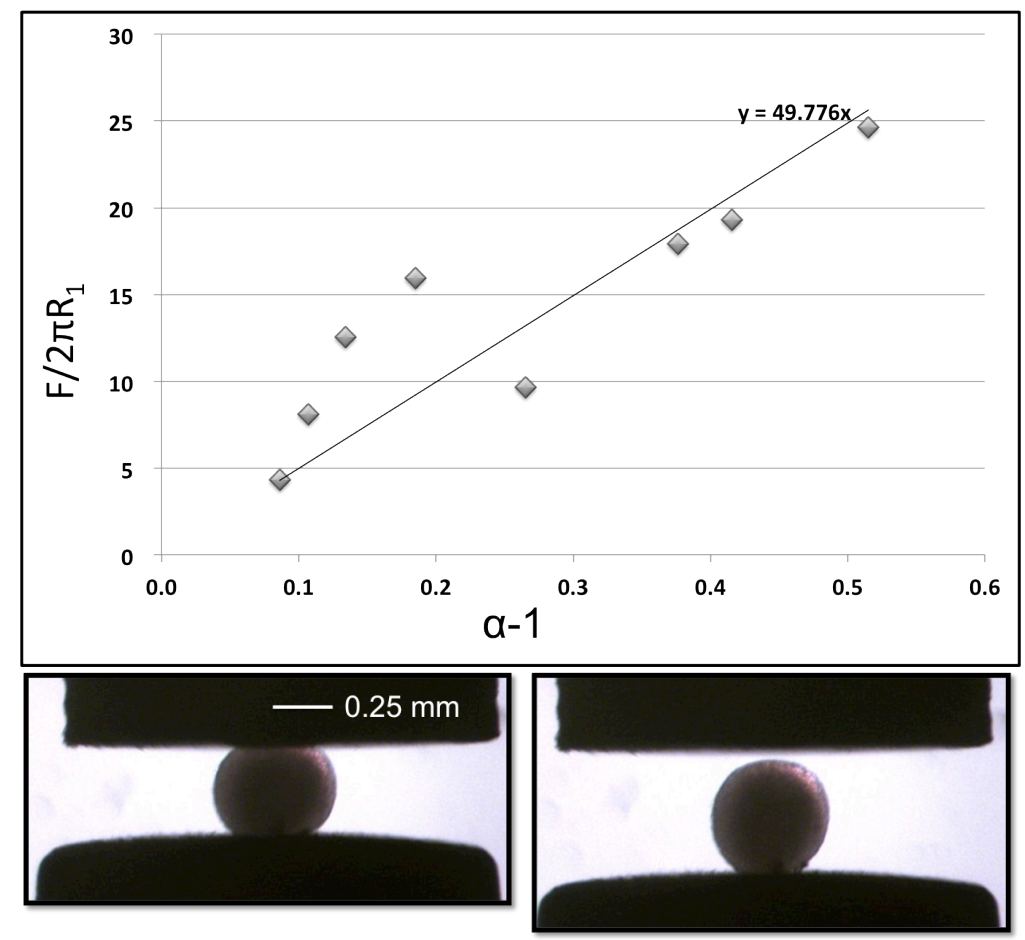

Figure 8. Upper panel: $\frac{\boldsymbol{F}}{2 \pi \boldsymbol{R}_{1}}$ vs. $\boldsymbol{\alpha}-\mathbf{1}$ and the linear fit to obtain the slope and consequently ATST of HSF aggregates. Lower panel: compressed and uncompressed HSF aggregates.

\section{Spheroids composed of Chinese Hamster Ovary cells}

The surface tension measurements for $\mathrm{CHO}$ aggregates, prepared with the EHAM method, were carried out the same way as for HSF aggregates described above. Table 2 provides the measured parameters for $\mathrm{CHO}$ aggregates and from these the values of $\alpha$, which are shown in the last column. Finally, the surface tension of $\mathrm{CHO}$ aggregates prepared by EHAM method is again obtained as the slope of the graph of $\left(F / 2 \pi R_{1}\right)$ versus $\alpha-1$. 


\begin{tabular}{|c|c|c|c|c|c|}
\hline Sample & Force (dyne) & $\mathrm{R}_{1}(\mathrm{~mm})$ & $\mathrm{H}(\mathrm{mm})$ & $\mathrm{H} /\left(2 \mathrm{R}_{1}\right)$ & $\alpha$ \\
\hline 1 & 0.127 & 0.341 & 0.536 & 0.786 & 1.102 \\
\hline 2 & 0.201 & 0.330 & 0.456 & 0.691 & 1.179 \\
\hline 3 & 0.222 & 0.323 & 0.467 & 0.722 & 1.151 \\
\hline 4 & 0.598 & 0.327 & 0.467 & 0.713 & 1.159 \\
\hline 5 & 0.691 & 0.324 & 0.504 & 0.778 & 1.107 \\
\hline 6 & 0.163 & 0.297 & 0.499 & 0.841 & 1.067 \\
\hline 7 & 0.875 & 0.351 & 0.460 & 0.654 & 1.216 \\
\hline 8 & 0.692 & 0.348 & 0.474 & 0.682 & 1.187 \\
\hline 9 & 0.372 & 0.340 & 0.474 & 0.698 & 1.173 \\
\hline 10 & 0.879 & 0.344 & 0.508 & 0.739 & 1.137 \\
\hline 11 & 0.823 & 0.343 & 0.372 & 0.541 & 1.366 \\
\hline 12 & 1.362 & 0.349 & 0.325 & 0.466 & 1.500 \\
\hline 13 & 1.597 & 0.372 & 0.384 & 0.517 & 1.405 \\
\hline 14 & 3.156 & 0.499 & 0.419 & 0.420 & 1.610 \\
\hline
\end{tabular}

Table 2. Equilibrium force, $\mathrm{R}_{1}, \mathrm{H}$ and $\alpha$ for $\mathrm{CHO}$ aggregates. 
The diamond symbols in Figure 9 correspond to the 14 individual measurements listed in Table 2 . The numerical value of the surface tension is given by the slope of the linear fit to the data points and yields $14.71 \mathrm{dyne} / \mathrm{cm}$. In addition based on the force relaxation diagram one can obtain the viscosity and shear modulus of the model tissue, respectively $\quad \eta=1.1 \times 10^{6}\left(\right.$ dyne. $\left.\mathrm{sec} / \mathrm{cm}^{2}\right) \quad$ and $G \approx 15 \times 10^{3}\left(\right.$ dyne $\left./ \mathrm{cm}^{2}\right)$.
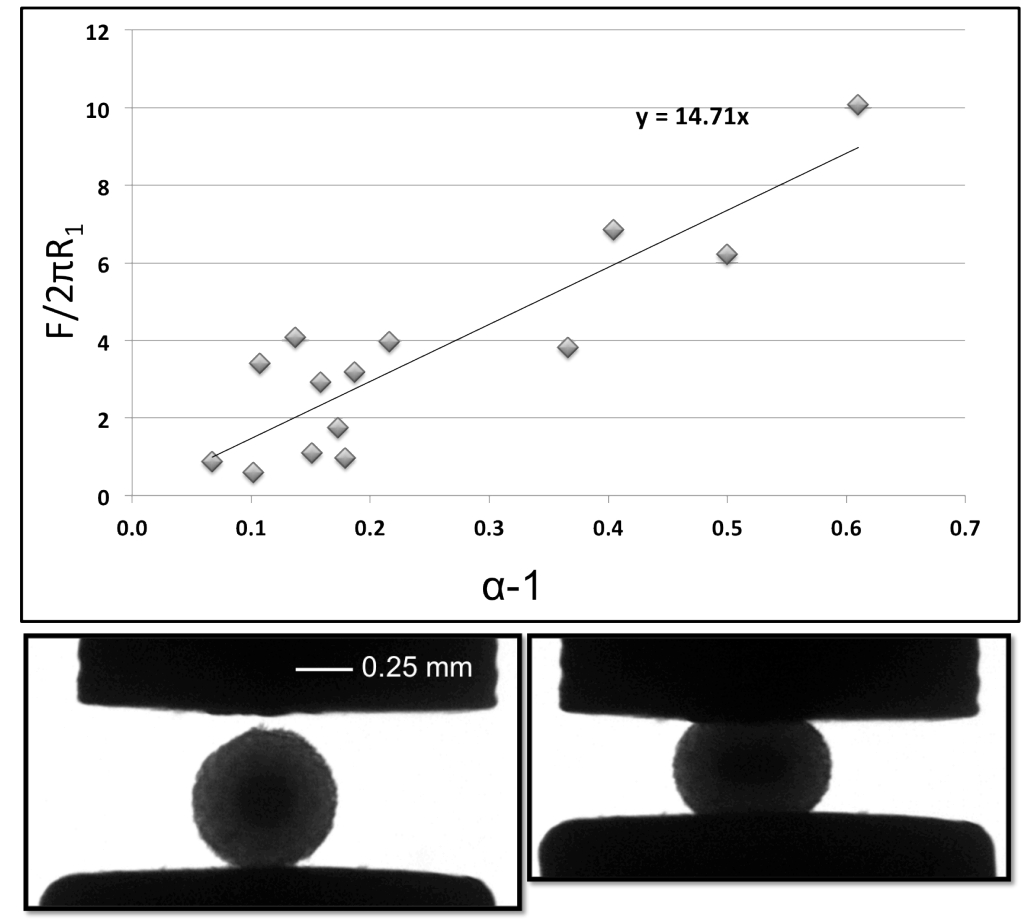

Figure 9. Upper panel: $\frac{\boldsymbol{F}}{2 \pi R_{1}}$ vs. $\boldsymbol{\alpha}-\mathbf{1}$ and the linear fit to obtain the slope and consequently ATST of CHO aggregates. Lower panel: compressed and uncompressed $\mathrm{CHO}$ aggregates. 


\section{Conclusions}

The apparent tissue surface tension, ATST, of two model tissue aggregates composed of $\mathrm{CHO}$ and HSF cells and prepared by the EHAM method was measured using the parallel plate surface tensiometry and evaluated by the exact solution of Laplace equation. We found for the ATST of the model tissues composed of $\mathrm{CHO}$ and HSF cell the respective values of $14.71 \mathrm{dyne} / \mathrm{cm}$ and $49.77 \mathrm{dyne} / \mathrm{cm}$, which implies the latter tissue to be the more cohesive one. The corresponding values for the viscosity and shear modulus of the model tissues composed of $\mathrm{HSF}$ and $\mathrm{CHO}$ cells were found to be respectively $\eta=1.7 \times 10^{6}\left(\right.$ dyne. $\left.\mathrm{sec} / \mathrm{cm}^{2}\right) \quad$ and $\quad G \approx 19 \times 10^{3}\left(\right.$ dyne $\left./ \mathrm{cm}^{2}\right)$ and $\eta=1.1 \times$ $10^{6}\left(\right.$ dyne. $\left.\mathrm{sec} / \mathrm{cm}^{2}\right)$ and $G \approx 15 \times 10^{3}\left(\right.$ dyne $\left./ \mathrm{cm}^{2}\right)$. 


\section{Chapter IV: Fusion of Spherical Aggregates}

In this chapter we discuss the fusion of spherical aggregates of similar and differing sizes prepared by the EHAM method. These experiments on one hand represent a model for tissue fusion (Pérez-Pomares \& Foty 2006), a fundamental early morphogenetic process, on the other hand serve to validate the CPD (Kosztin et al. 2012).

The fusion of spheroids composed of highly viscous material (such as cellular material) has been a subject of numerous studies (see references e.g. Kosztin et al, 2012, in particular Frenkel1945). Here we rely on these early studies, but also use their extension to incorporate situations not discussed before, but highly relevant to the system under study. In particular we will consider the case when the volume of the fusing aggregates varies, as we have observed in our experiments. First we present our studies for $\mathrm{CHO}$ cell aggregates, and then discuss the case of HSF aggregates.

\section{Theory}

Fusion experiments involve two aggregates of either equal or unequal size, placed contiguously to each other. Because of the apparent liquid properties of such cellular spheroids, the end result of their fusion is a 
single spheroid, a configuration with minimal area. As originally pointed out by Frenkel (Frenkel 1945) and later generalized by others (Eshelby 1949; Kosztin et al. 2012), the fusion of two contiguous cell aggregates (i.e. two highly viscous spheroids) is driven by surface tension $\gamma$ and resisted by viscosity $\eta$.

Here we briefly introduce the theory background for fusing aggregates as developed by Kosztin and co-workers (Kosztin et al. 2012; McCune et al. 2014). The two fusing aggregates were modeled as two circular arcs of radius $R(\theta)$ with circular contact region of radius $r(\theta)=R(\theta) \sin \theta$ (Fig. 10). The fusion angle is defined in Figure 10. At first, cell aggregates were considered to be composed of incompressible material, in which mass is conserved and the volume is constant over the fusion process (Kosztin et al. 2012).

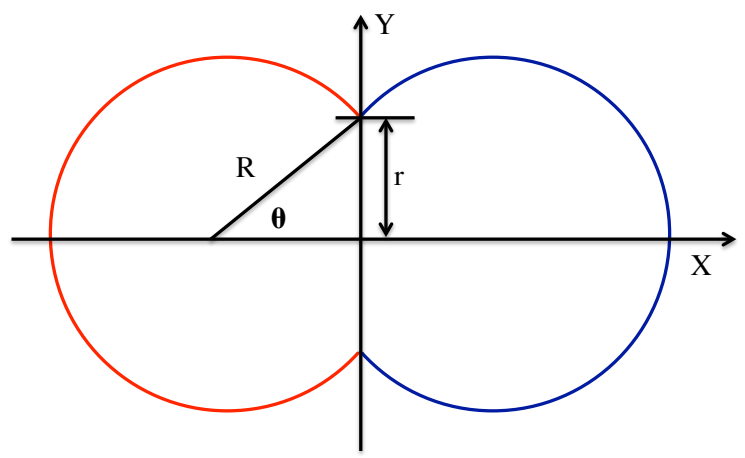

Figure 10. $R, r$ and the fusion angle for two identical fusing spherical aggregates. 
$R_{0}$ is the radius of the aggregates prior to the initiation of fusion (i.e. for $\theta=0$ ). The time evolution of the fusion process is described by the fusion angle $\theta=\theta(t)$. Fusion is complete when the two aggregates coalesce into one, corresponding to the fusion angle reaching the value of $90^{\circ}$. Since flow rate in the viscous multicellular system, undergoing shape relaxation is slow, Kosztin and collaborators assumed that the rate of decrease of surface area is equal to the rate of energy dissipation due to viscosity. Furthermore, for a homogeneous biaxial extensional flow during fusion the strain rate tensor is constant and diagonal (Eshelby 1949; Bellehumeur et al. 1998). With these details, Kosztin and collaborators developed an equation for $\theta=\theta(t)$ (for details see Kosztin et al. 2012). In terms of the characteristic fusion time $\tau$ defined by

$$
\tau=\eta R_{0} / \gamma,
$$

the solution of this equation was found as

$$
\left(\frac{r}{R}\right)^{2}=\sin ^{2} \theta \approx 1-\exp (-t / \tau)
$$

For $t \ll \tau$, Eq. (4.2) reduces to the linear expression $\left(\frac{r}{R}\right)^{2} \approx\left(t-t_{0}\right) / \tau$ obtained by Frankel (1945) and Eshleby (1949). Here $t=0$ denotes the moment when the two aggregates are contiguously deposited and $t_{0}$ in 
exponent is a fitting parameter, that represents the time of the de facto start of fusion.

Once the experiments, to be described below have been carried out it was found that the original assumption on the volume conservation of the aggregates in the course of fusion is not fulfilled. Thus the original theory had to be extended to include the case of nonconserved volume (for details see (McCune et al. 2014)).

In brief, one assumes that the decrease in volume can be described by the decrease of the radius of the aggregate as $R_{0}(t)=$ $a(t) R_{0}(0)$, with the dimensionless quantity $a(t)<1,(a(0)=1)$. For the analysis of the experiments the following form of $a(t)$ was assumed

$$
a(t)=a_{0}+\left(1-a_{o}\right) \exp (-\lambda t) .
$$

Here $a_{0}=a(\infty) \leq 1$ is the relative change of the linear dimension of the system during its complete volume relaxation, and $\lambda$ is the rate of this relaxation (McCune et al. 2014).

We also extended the theory to the fusion of different size cell aggregates (McCune et al. 2014). Again first we considered the case with constant volume and then generalized the theory to the case of non-constant volume (McCune et al. 2014). 
The fusion of unequal size (uneven) aggregates were modeled as two circular arcs of radii $R_{1}\left(\theta_{1}\right)>R_{2}\left(\theta_{2}\right)$ with a circular contact "neck" region of radius (Figure 11 )

$$
r=R_{1} \sin \theta_{1}=R_{2} \sin \theta_{2} .
$$

The fusion angles $\theta_{1,2}$ are defined in (Figure 11). The initial radii of the uneven aggregates are $R_{i 0}=R_{i}(0), i=1,2$. As without the loss of generality we take $R_{1}\left(\theta_{1}\right)>R_{2}\left(\theta_{2}\right)$, has, $b=\frac{R_{10}}{R_{20}}>1$.

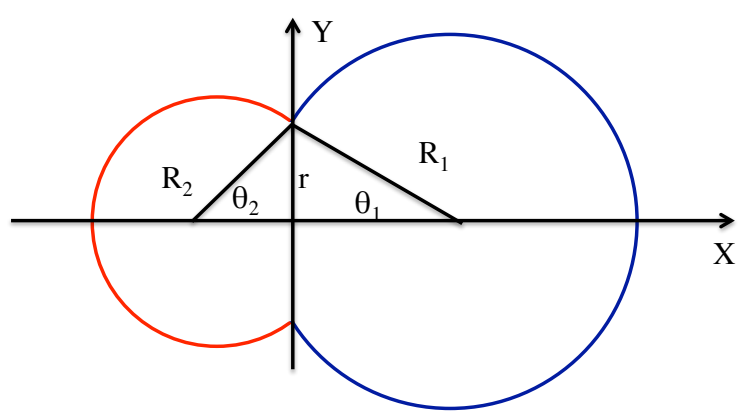

Figure 11. Radii and fusion angles for the fusion of two different size aggregates.

The dynamics of the fusion process is again determined by the time dependence of the fusion angles $\theta_{1}(t)$ and $\theta_{2}(t)$ (they vary from $\theta_{1}(0)=\theta_{2}(0)=0$ to $\theta_{1}(\infty)=\theta_{\max }$ and $\left.\theta_{2}(\infty)=\pi-\theta_{\max }\right)$, (for details see (McCune et al. 2014))

$$
\tau_{\text {unequal }}=\eta R_{10} / \gamma
$$


For the case of non-constant volume the decrease in the radii of the aggregates was again represented by $R_{i 0}(t)=a(t) R_{i 0}(0), i=$ 1,2 , where $a(t)$ is a time dependent dimensionless quantity (McCune et al. 2014).

In the experiments reported below $a(t)$ was approximated by the same exponential expression as given in Eq. 4.3 above.

Finally, for the time dependence of the fusion angles one can introduce the functions $q(t)=\sin ^{2} \theta_{i}(t)$ and $q_{0}(t)=\sin ^{2} \theta_{i}(t)$ respectively for the non-volume-conserving and volume-conserving case (McCune et al. 2014). The experimental results showed (see below) that for $a(t)<1$, corresponding to the decrease in the volume of the fusing aggregates, the function $q(t / \tau)$ could be well approximated with the volume conserving result, $q_{0}\left(t / \tau_{e f f}\right)$, albeit with a smaller effective characteristic fusion time, $\tau_{e f f}=c_{a} \tau<\tau$

$$
q(t / \tau) \approx q_{0}\left(t / c_{a} \tau\right), \quad 0<a_{0}<c_{a}<1
$$

\section{Experiments}

Fusion experiments were carried out using spherical cell aggregates prepared by the EHAM method as described in Chapter II. The time evolution of fusion was recorded with a Cannon digital camera attached to an Olympus IX70 inverted microscope (Figure 12). 


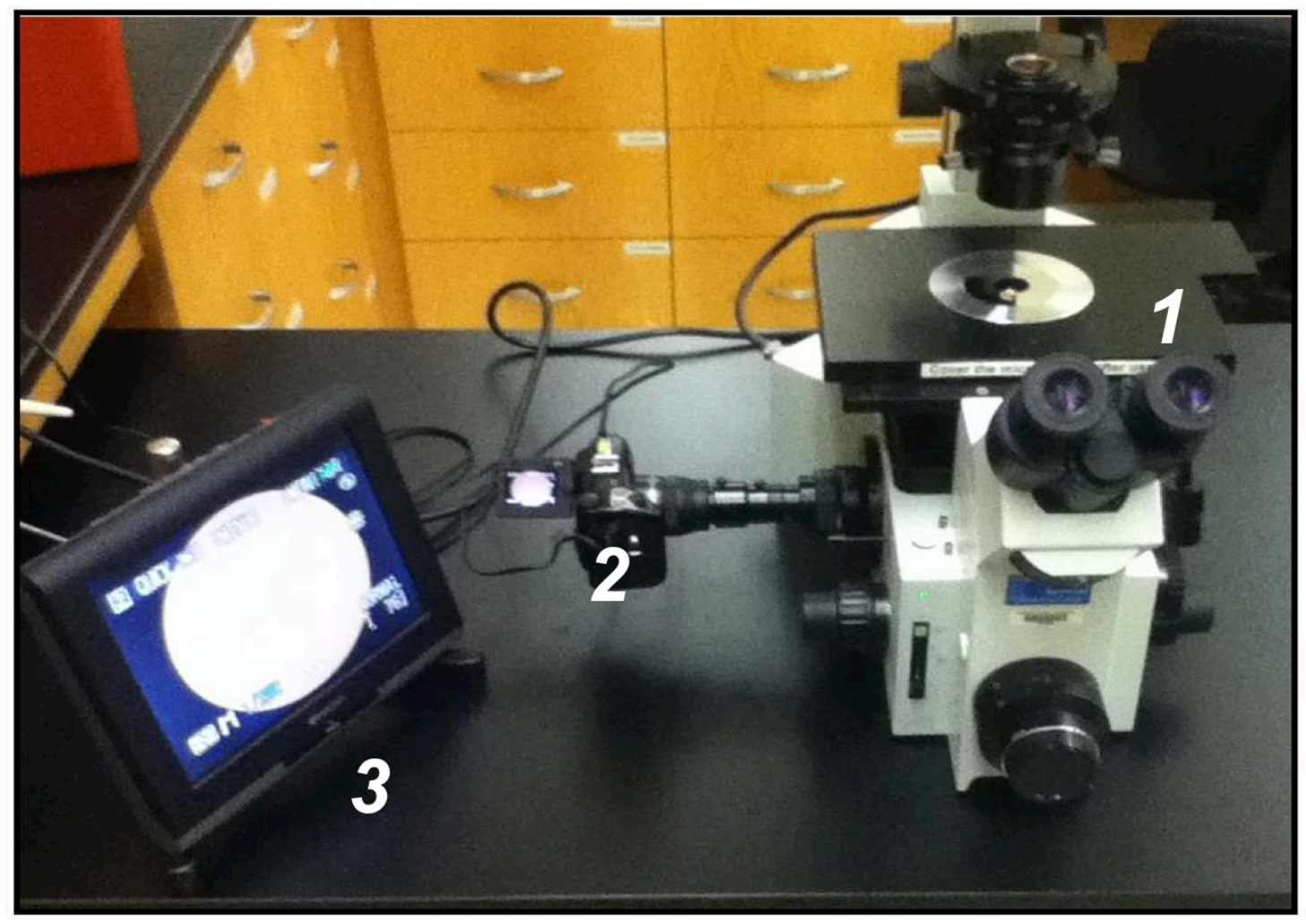

Figure 12. The fusion workstation, an Olympus IX70 inverted microscope (1) attached to Cannon digital camera (2) attached to a monitor (3) through which fusing aggregates can be observed.

The shape evolution of the aggregates was investigated by analyzing the recorded images using the software Mathematica (Wolfram, Mathematica 9, Champaign, IL). Two circular arcs were fitted to the fusing aggregates and the quantities $R$ and $\Theta$, for equal size aggregates (see Figure 10 ) and $R_{1,2}$ and $\Theta_{1,2}$ for the unequal size aggregates (see Figure 11) were determined. This was followed by plotting $\sin ^{2}(\Theta)$ versus time to find the characteristic fusion time $\tau$ (see Eq. 4.1, 4.2 and 5). 


\section{Fusion of CHO spheroids: the initial linear regime}

It was shown by Frenkel that the initial phase of fusion of two highly viscous liquid drops could accurately be described by a linear relationship in time (Frenkel 1945). That implies that the area of the interfacial ring formed by the two fusing drops increases linearly in time (for details see (Kosztin et al. 2012)). In order to determine how good this linear fit is, in the case of $\mathrm{CHO}$ aggregates, prepared with the EHAM method, we investigated the fusion $\tau$ in two different ways: using this initial linear fit, $\left(\frac{r}{R}\right)^{2} \approx\left(t-t_{0}\right) / \tau$ (up to 38 hours of fusion) and the full exponential fit, Eq. 4.2 for the full fusion process (followed up to a week). In the case of the linear fit, we followed the fusion process by recording the images of the aggregate pairs each two hours up to 26 hours and then taking a last image after $38 \mathrm{hrs}$. 


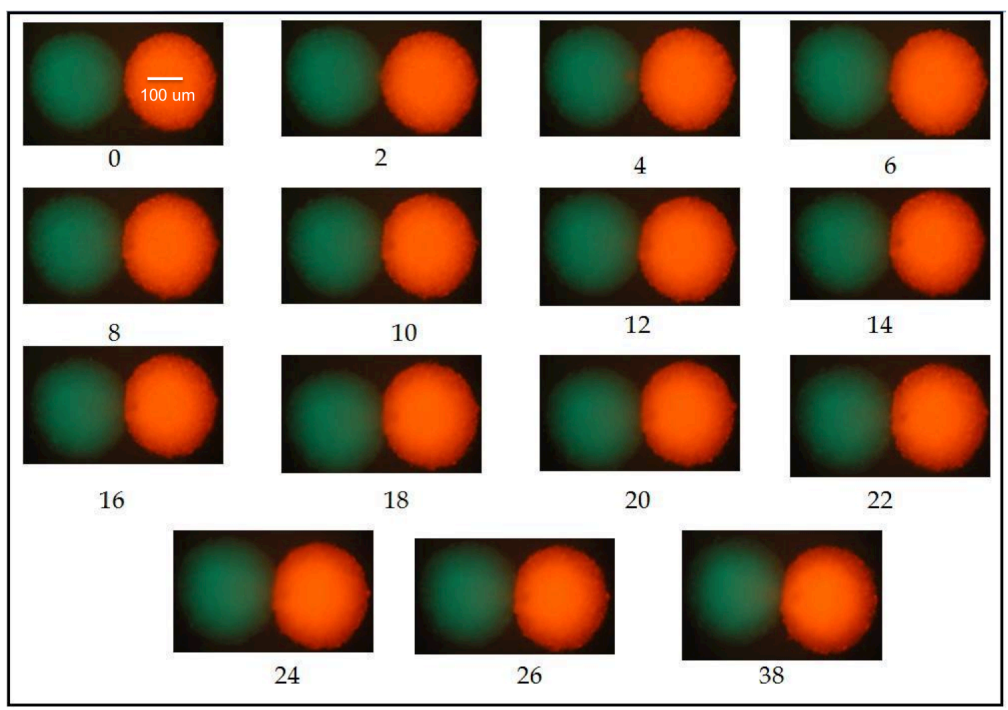

Figure 13. Fusion of equal size $\mathrm{CHO}$ aggregates (numbers denote time in hours). Special care was taken to record the shape of the fusing spheroids. In order to overcome the possible shaking of the construct (and thus making the recorded images inconsistent), the microscope was placed very close to the incubator, where the aggregates were kept between the recordings. This way the time needed to carry the Petri dish containing the aggregates from the incubator to the microscope was minimized.

Figure 13 presents the images for the fusing $\mathrm{CHO}$ aggregates for the first $38 \mathrm{~h} .8$ pairs of aggregates of equal size have been used for this experiment and analyzed to find the characteristic fusion time $\tau$.

The graph $(r / R)^{2}$ vs. time, was fitted with the linear fit and $\tau$ was calculated for each sample. With this procedure the average fusion time calculated over 8 experiments with equal size $\mathrm{CHO}$ aggregates, was found to be $\tau=163.15 \pm 28$ hours. Figure 14 shows the variation of the fusion time across the experiments. 


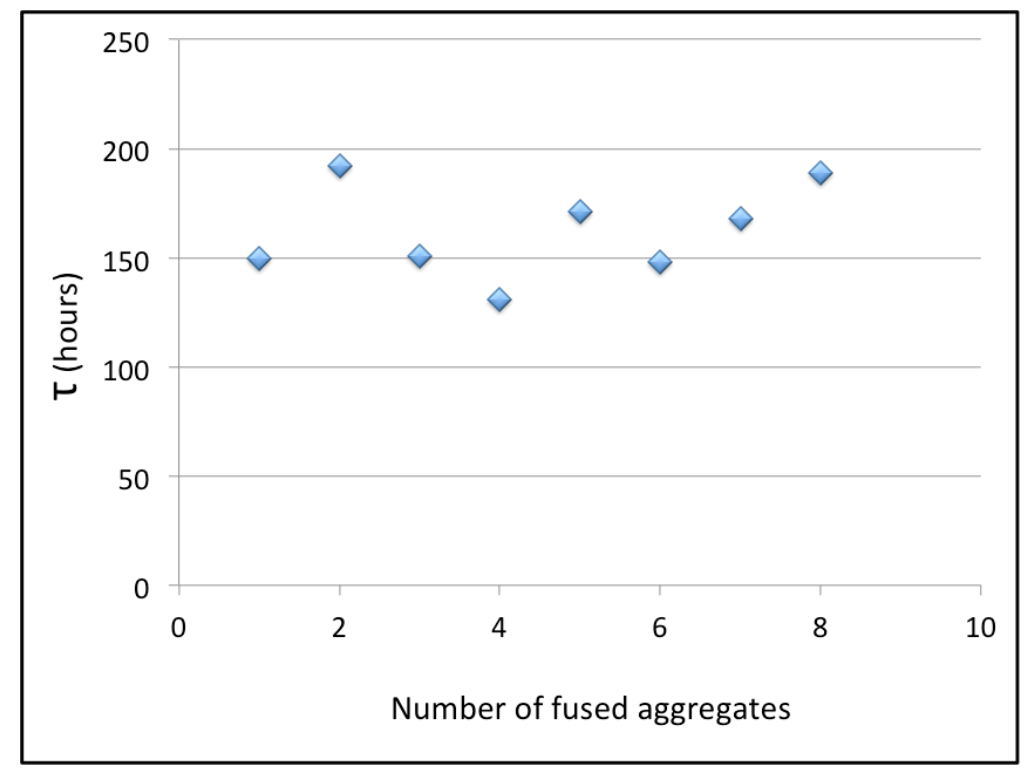

Figure 14. Variation of fusion time for $\mathrm{CHO}$ aggregates prepared with the EHAM method as obtained from the linear fit to the initial linear regime of fusion.

\section{Fusion of CHO spheroids: the complete process}

In another set of experiments the fusion of two equal size $\mathrm{CHO}$ aggregates was followed for an entire week and recorded at times 0 , $2,5,8,11,14,17,25,29,33,37,39,48,54,61,73,85,97,109$, $121,133,145,157$ and 173 hours. This time interval includes the portion of the fusion process that is beyond the linear part. Figure 15 shows the distribution for the characteristic fusion time over 8 samples. 


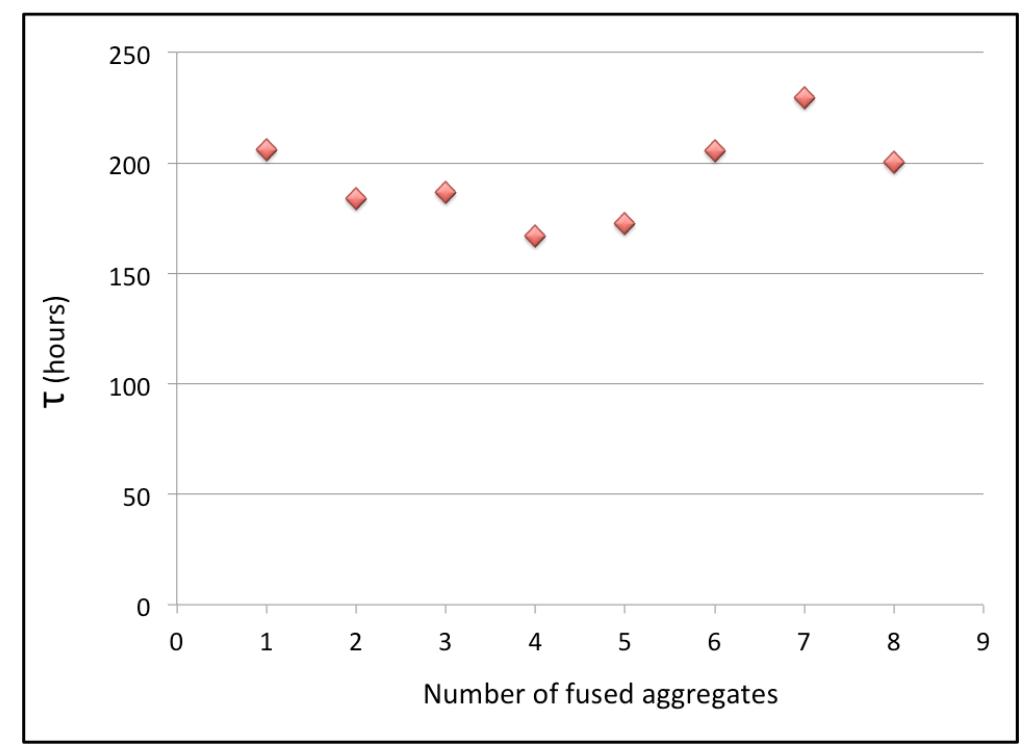

Figure 15. Distribution of the characteristic fusion times for $8 \mathrm{CHO}$ samples prepared by EHAM as obtained from the exponential fit to the fusion experiments recorded for a week.

The average characteristic fusion time as can be obtained from the data in Figure 15 is $193.23 \pm 36$ hours, which is about $20 \%$ larger than the value $163.15 \pm 28$ hours obtained from the linear fit to the initial fusion regime.

\section{Fusion of fibroblast spheroids (same and different size)}

The fusion experiment performed with HSF aggregates prepared by EHAM was followed for a week. Figure 16 and 17 show the shape of the aggregates at the start of fusion, respectively for equal and unequal size aggregates. Figure 18 shows uneven fusing aggregates at selected instances in the process. The fusion time for the uneven size 
aggregates can be found using the expression $\tau_{\text {uneven }}=$ $\left(\tau_{\text {even }} / R_{0 \text { even }}\right) R_{10}$, where $R_{10}$ is the initial radius of the larger aggregate and $\mathrm{R}_{0 \text { even }}$ and $\tau_{\text {even }}$ are obtained from the experiments with equal size aggregates. Here "Time $=0$ " is the moment that the aggregates are placed close to each other. Note that "Time $=0$ " is different from " $t_{0}$ " which is a fitting parameter and shows the actual starting point of fusing.

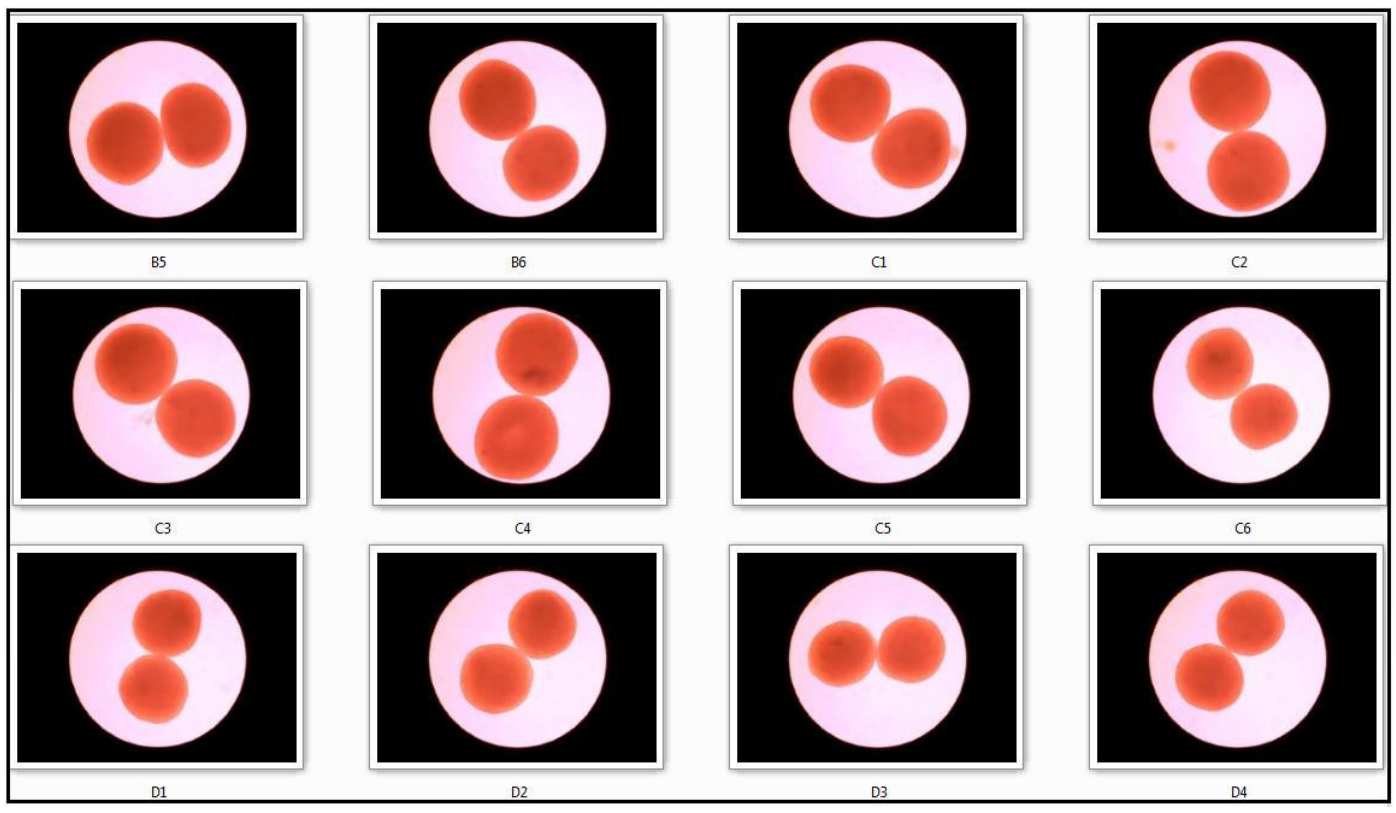

Figure 16. Time $=0$ (placing aggregates close to each other) for 12 pairs of equal size aggregates. 


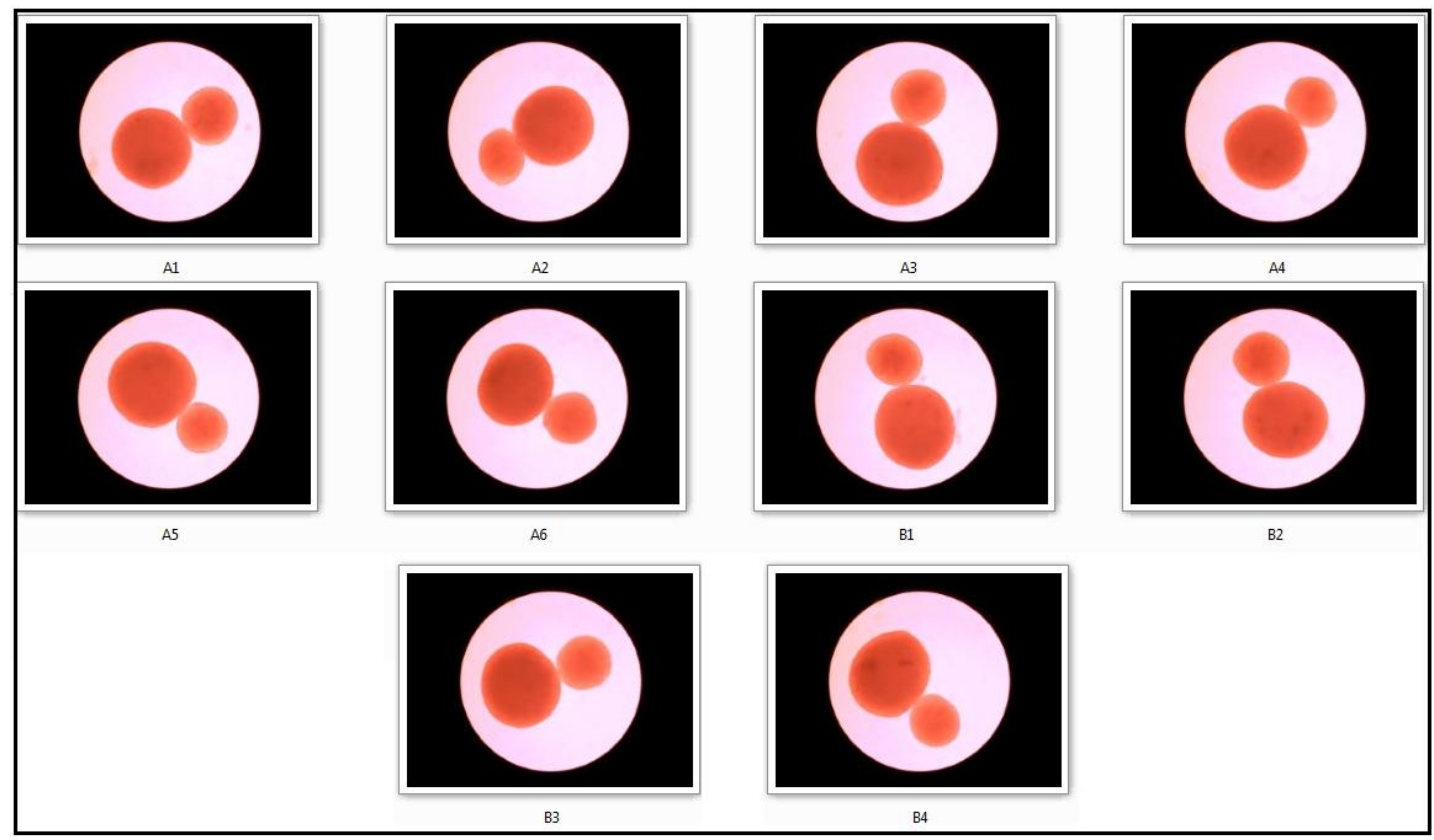

Figure 17. Time $=0$ (placing aggregates close to each other) for 10 pairs of unequal size aggregates.

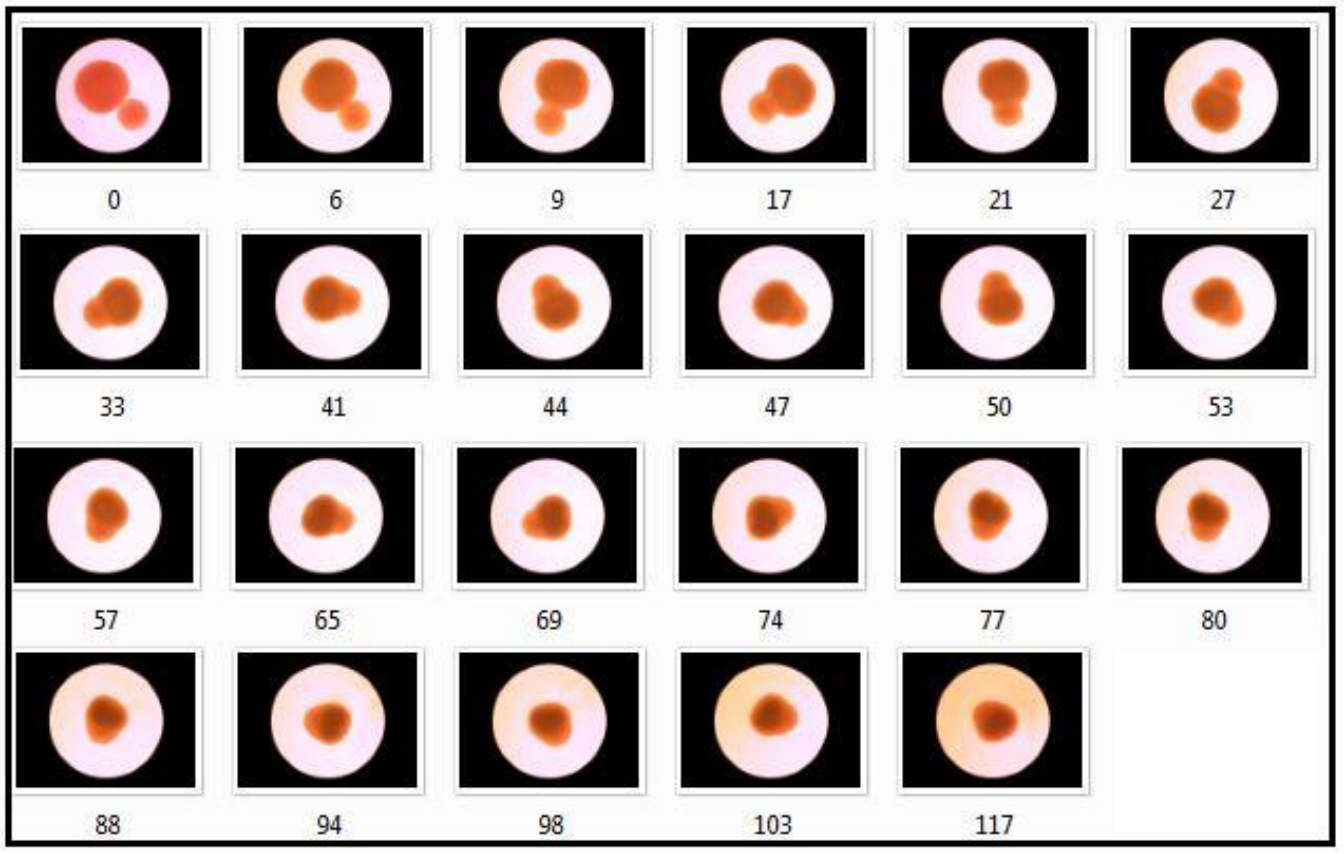

Figure 18. Recording of shape evolution in a typical fusion experiment with unequal size aggregates, as followed for several days (numbers denote time in hours). 


\section{Analysis of the fusion experiments: equal size aggregates}

Contours fitted to the recorded images during the fusion of two identical HSF aggregates, with initial radius $R_{0}=274 \mu \mathrm{m}$, are shown in

Figure 19. Analysis of the images, by considering the change in volume, resulted in $\tau_{e f f}=40.8 \tau=48.0 \mathrm{~h}, t_{0}=9.3 \mathrm{~h}, a_{0}=0.73$ and $\lambda^{-1}=50 \mathrm{~h}$.
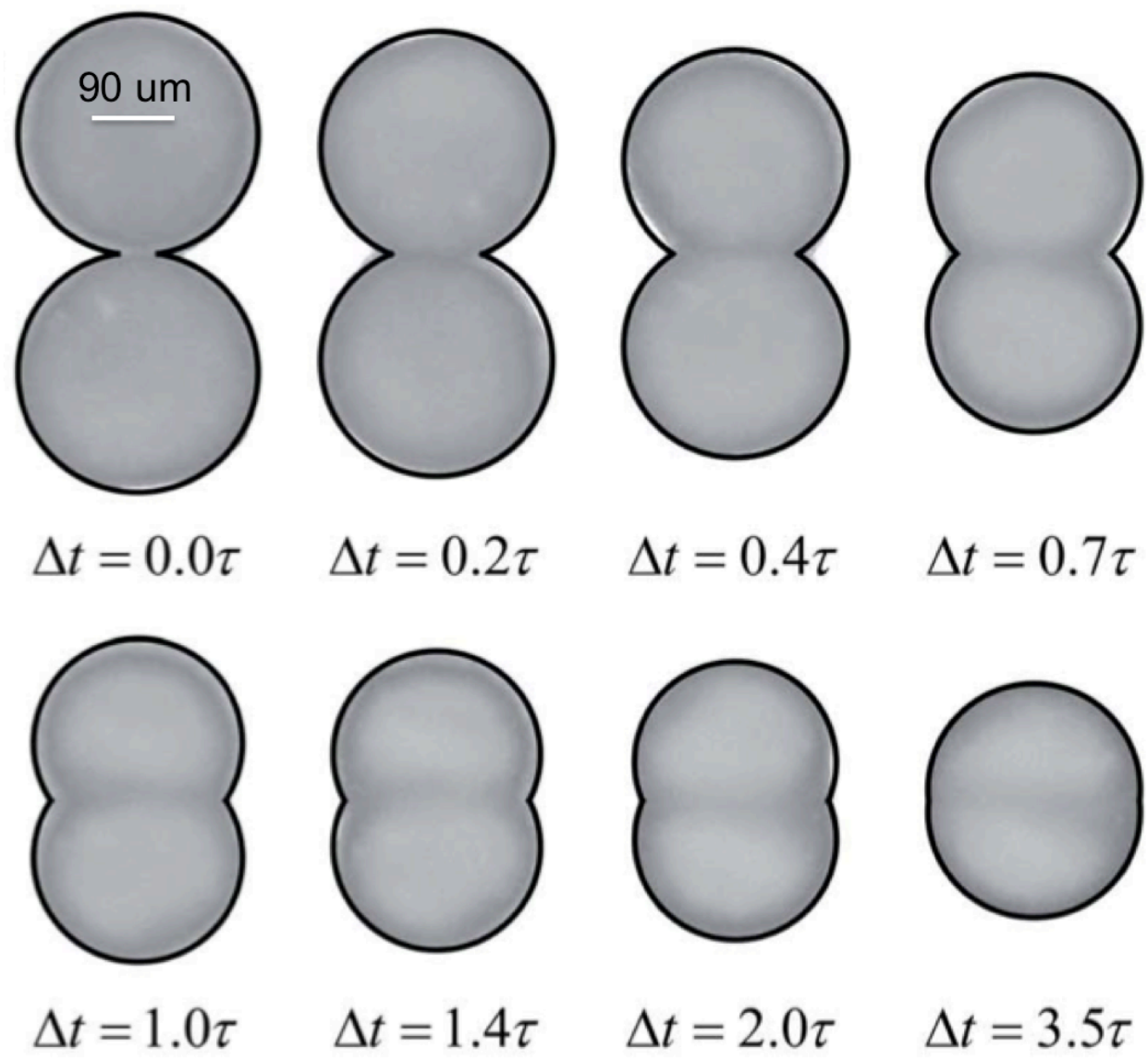

Figure 19. Rocording of shape evolution in a typical fusion of two equal sized HSF aggregates of $R_{0}=274$ micron. The solid-line contours represent the theoretical shapes of the fusing aggregates. 
As noted earlier, despite the sizable decrease in the volume of the system of fusing aggregates $\left(V(\infty) / V\left(t_{0}\right)=a_{0}^{3} \approx 0.4\right)$, the difference between the theoretical fitting functions $q(\Delta t / \tau)$ and $q_{0}(\Delta t / \tau)$, (respectively the solid and dashed curves in Figure 20) is small. In fact, in agreement with Eq. 4.6, $q(\Delta t / \tau)$ is almost indistinguishable from $q_{0}\left(\Delta t / c_{a} \tau\right)$ with $c_{a}=\left(1+a_{0}\right) / 2=0.87$ (dashed-dotted line in Figure 20). Based on this result, one may conclude that, in general, experimental fusion data for cell aggregates with decreasing system volume can be analyzed (and predicted) quantitatively using the constant volume results provided that the characteristic fusion time in the volume conserving case is replaced with $\tau_{e f f}=c_{a} \tau<\tau$. 


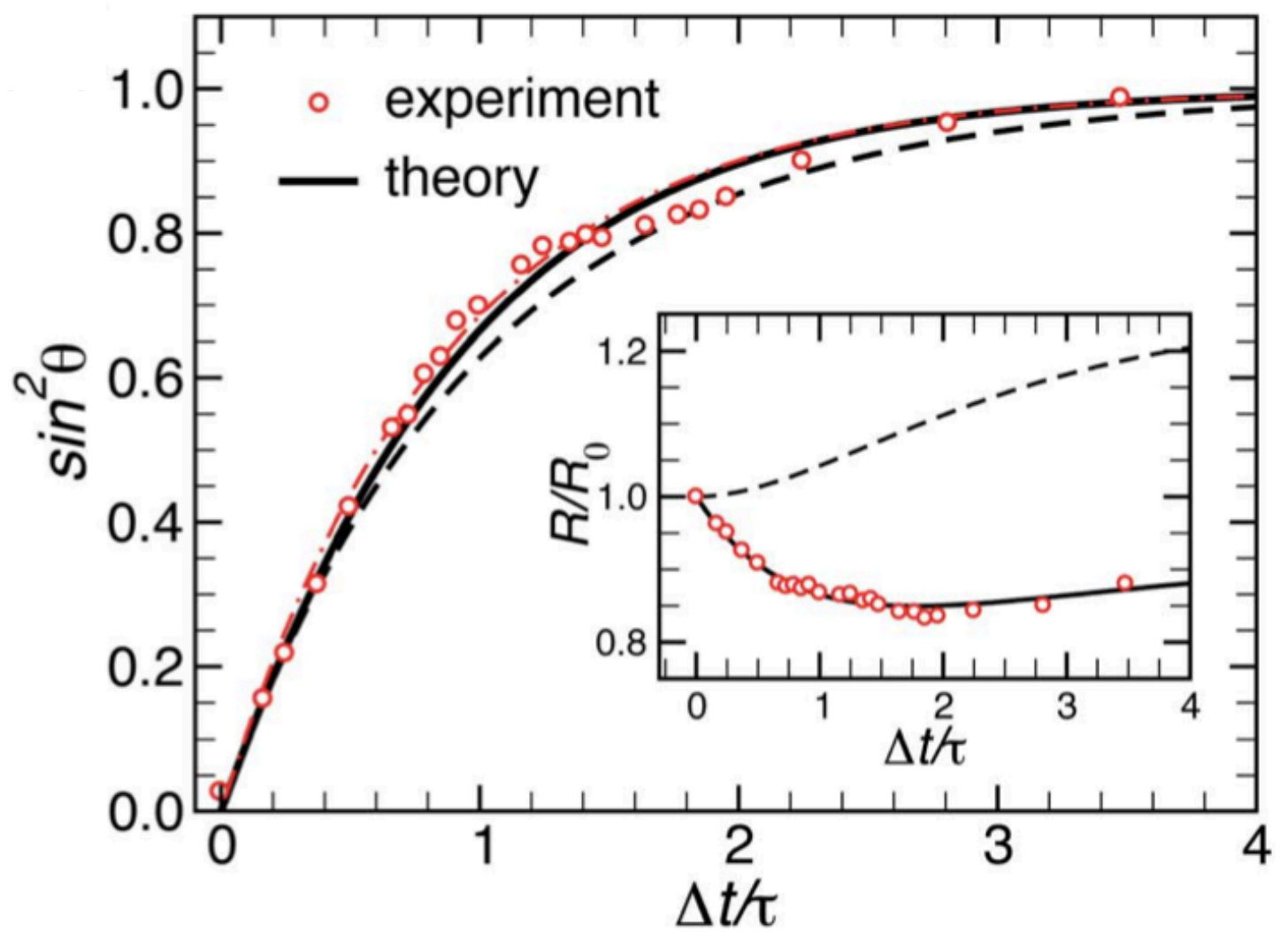

Figure 20. Theoretical fit (solid curve) to the experimental data (open circles) for $\sin ^{2} \Theta$ vs. $\Delta t / \boldsymbol{\tau}$ for HSF aggregates of equal size corresponding to Figure 19. The dashed (dashed-dotted) curve represents the volume conserving theoretical result, with the same (effective) characteristic fusion time. Inset: time dependence of $R(t) / R_{0}$. The broken upper curve shows the variation of the ratio for the case of conserved volume. The strong deviation from the volume conserving upper curve, as shown in the lower curve (open circles: experimental results; solid curve: theoretical result using $R_{0}(\mathrm{t})=a(t) R_{0}(0)$ with $a(t)$ given in Eq. 4.3) implies a substantial decrease in volume during fusion.

\section{Analysis of the fusion experiments: unequal size aggregates}

Figure 21 shows the snapshots of the fusion of two unequal HSF aggregates, with the initial radii $R_{10}=302 \mu \mathrm{m}$ and $R_{20}=178 \mu \mathrm{m}$ and ratio of radii equal to $b=R_{10} / R_{20}=1.7$. To analyze the snapshots of the 
fusing unequal aggregates again Mathematica was used: the contours of the fusing aggregates were fitted with (unequal) circular arcs as shown in Figure 21.
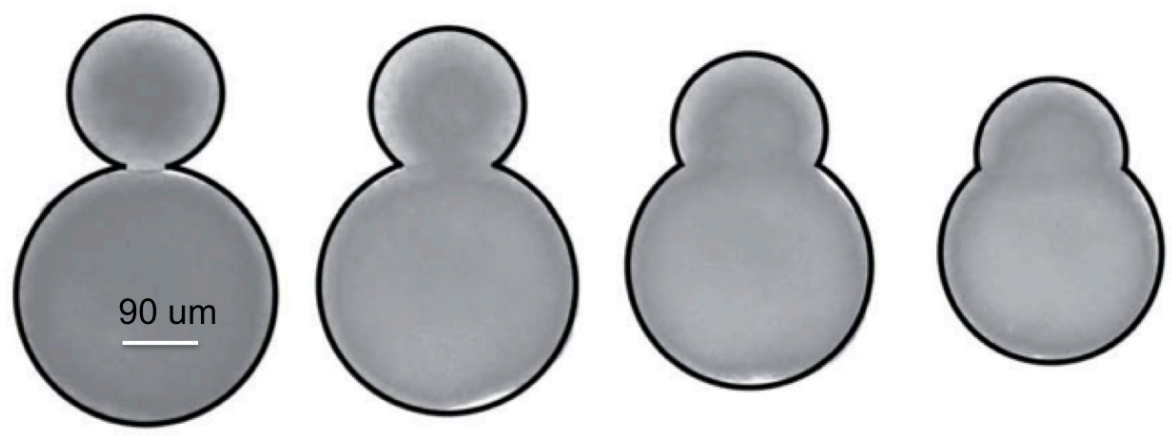

$$
\Delta t=0.1 \tau
$$
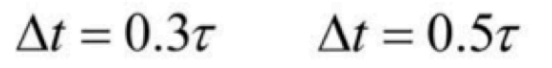

$\Delta t=0.9 \tau$
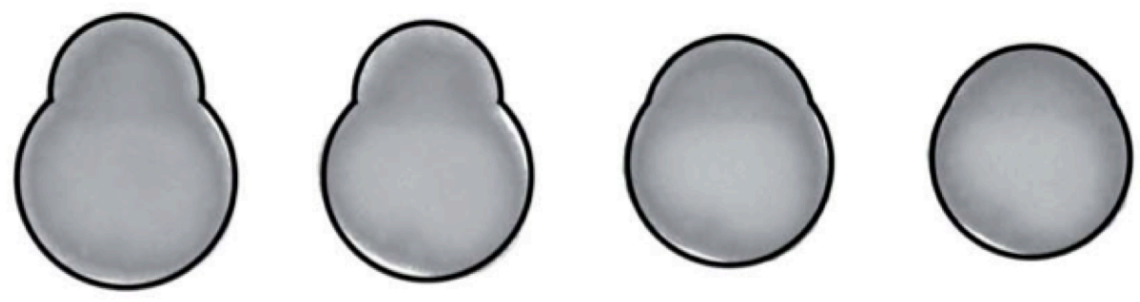

$$
\Delta t=1.1 \tau
$$

$$
\Delta t=1.4 \tau
$$

$$
\Delta t=2.2 \tau
$$

$$
\Delta t=3.0 \tau
$$

Figure 21. Recording of shape evolution in a typical fusion experiment of two unequal HSF aggregates.

Analysis of the images using the theoretical model resulted in $\tau_{\text {eff }}=40.8, \tau=48.9 \mathrm{~h}, a_{0}=0.67$ and $\lambda^{-1}=52.6 \mathrm{~h}$. 
Similarly to the fusion of equal aggregates: (i) the difference between the theoretical fits $q(\Delta t / \tau)$, corresponding to volume change with $a_{0}=0.67$ (solid curves in Figure 22), and $q_{0}(\Delta t / \tau)$, corresponding to constant volume (dashed curves in Figure 22) is relatively small; and (ii) $q_{0}\left(\Delta t / c_{a} \tau\right)$ (dashed-dotted curve in Figure 22) with $c_{a}=0.87$ approximate well $q(\Delta t / \tau)$.

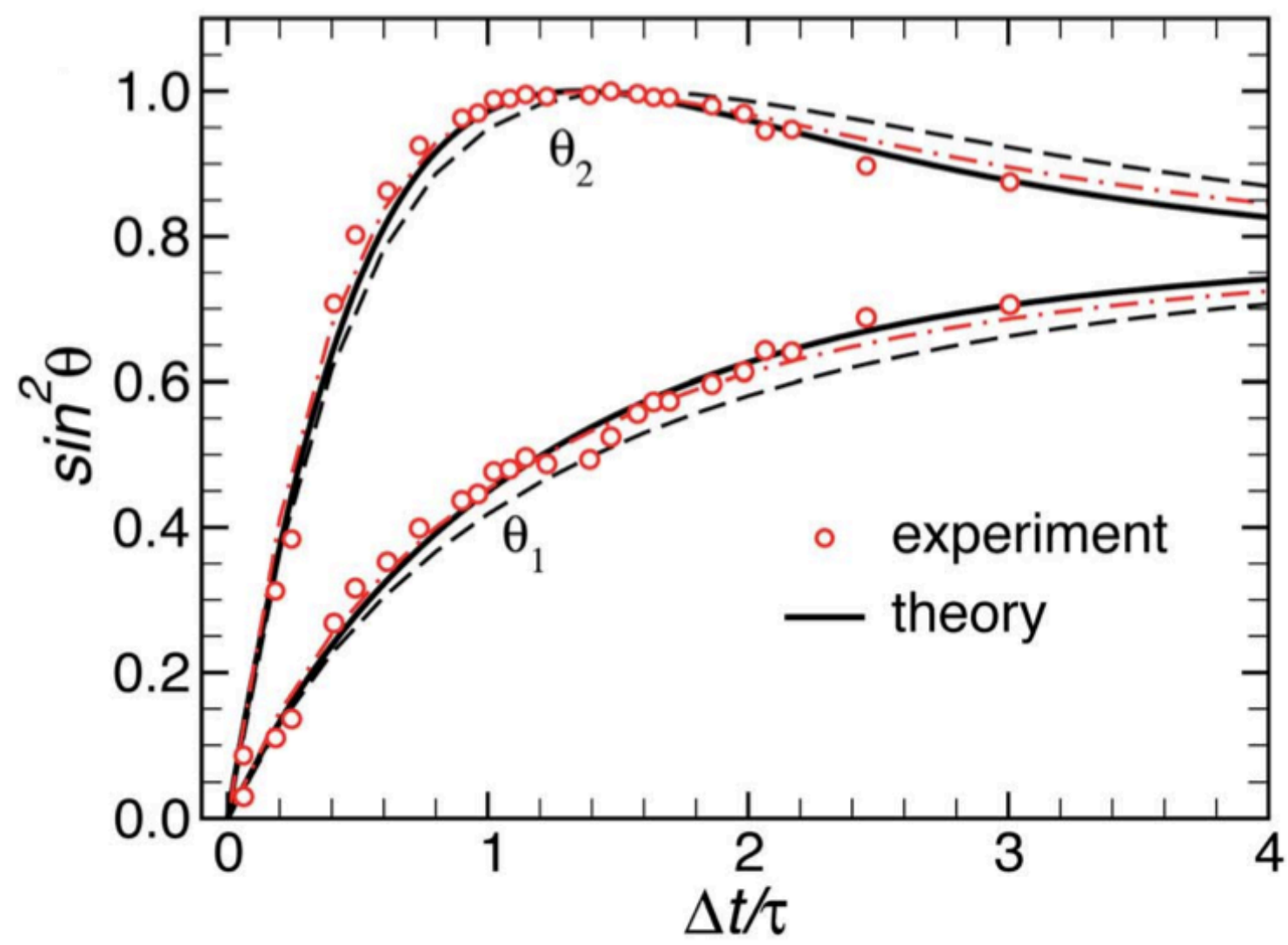

Figure 22. Theoretical fit (solid curve) to the experimental data (open circles) for $\sin ^{2} \Theta$ vs. $\Delta t / \tau$ for HSF aggregates of unequal size, corresponding to Figure 21. The dashed (dashed-dotted) curve represents the volume conserving theoretical result, with the same (effective) characteristic fusion time. 
A few remarks on the above results are due at this point.

(i) The fact that we obtained essentially the same values for the volume change parameter, $a_{0} \approx 0.7$, and the volume relaxation rate, $\lambda=0.02 h^{-1}$, in both fusion experiments (equal and unequal HFS spherical aggregates), suggests that the time dependence of the volume relaxation of a multicellular system depends primarily on the used cell type and preparation method.

(ii) The change in volume required the modification of the theory, which was originally developed for conserved volume in the course of fusion. Considering the change in volume through additional fitting parameters, i.e. $a_{0}$ and $\lambda$ based on Equation 4.3 , the experimental results could accurately be described by the theory. As Figure 22 reveals during the fusion of unequal size aggregates, the instantaneous radius of the smaller aggregate initially changed faster than that of the larger aggregate. It increases to a maximum and then decreases towards the radius of the resulting final spheroid. At the same time the instantaneous radius of the larger aggregate converges from below to the final radius. These variations are accurately described by the theory.

(iii) Using $a_{0} \approx 0.7$ and $c_{a} \approx 0.85$, one can obtain the characteristic fusion times from both measured $\tau_{e f f}$ for even and uneven aggregates 
equal to $\tau_{\text {even }}=48$ hours and $\tau_{\text {uneven }}=48.9$ hours for the case of Figure 19 and 21.

(iv) The theoretical prediction for the fusion of unequal size aggregates (based on the result for equal size aggregates, equations 4.1 and 4.5 ) predicts $\tau=52.9$ hours for the characteristic fusion time of uneven aggregates while the experimental result showed 48.9 hours for the case of Figure 21 . These values differ by $\sim 10 \%$, which, considering the numerous sources of errors when working with cellular aggregates, could be considered as excellent agreement and thus a strong confirmation of the modified theory. Table 3 summarizes the data for fusion of four equal size samples. The data for fusion of three unequal size aggregates are summarized in Table 4. Each equal size fusing aggregate can be used as the calibrating experiment and predict the fusion time for each unequal size fusing aggregates. In this case we can have 12 predictions for three unequal size aggregates that are summarized in Tables 5-7. 


\begin{tabular}{|l|l|l|l|l|l|l|}
\hline Sample & $R_{0}(\mu \mathrm{m})$ & $\tau_{\text {eff }}(\mathrm{hrs})$ & $\mathrm{a}_{0}$ & $\mathrm{C}_{\mathrm{a}}$ & $\tau(\mathrm{hrs})$ & $\tau / R_{0}(\mathrm{hrs} / \mu \mathrm{m})$ \\
\hline 1 & 228 & 44.8 & 0.79 & 0.90 & 49.7 & 0.21 \\
\hline 2 & 230 & 38.4 & 0.79 & 0.90 & 42.66 & 0.18 \\
\hline 3 & 231 & 40.3 & 0.74 & 0.87 & 46.32 & 0.20 \\
\hline 4 & 276 & 41.02 & 0.70 & 0.85 & 48.25 & 0.17 \\
\hline
\end{tabular}

Table 3. Fusion results for 4 even HSF aggregate

\begin{tabular}{|l|l|l|l|l|l|l|}
\hline Sample & $R_{10}(\mu m)$ & $R_{20}(\mu m)$ & $\tau_{\text {eff }}(\mathrm{hrs})$ & $\mathrm{a}_{0}$ & $\mathrm{C}_{\mathrm{a}}$ & $\tau(\mathrm{hrs})$ \\
\hline 1 & 283 & 186 & 45.76 & 0.70 & 0.85 & 53.8 \\
\hline 2 & 286 & 168 & 41.28 & 0.71 & 0.855 & 48.28 \\
\hline 3 & 273 & 181 & 48.8 & 0.71 & 0.855 & 57.07 \\
\hline
\end{tabular}

Table 4. Fusion results for 3 uneven HSF aggregates 


\begin{tabular}{|l|l|l|l|l|}
\hline $\begin{array}{l}\tau / R_{0}(\mathrm{hrs} / \mu \mathrm{m}) \\
\text { Even sample }\end{array}$ & $R_{10}(\mu \mathrm{m})$ & $\begin{array}{l}\tau_{\text {uneven }}(\mathrm{hrs}) \\
\text { Prediction }\end{array}$ & $\begin{array}{l}\tau_{\text {uneven }}(\mathrm{hrs}) \\
\text { Experiment }\end{array}$ & \% Error \\
\hline 0.21 & 283 & 59.43 & 53.8 & 10 \\
\hline 0.18 & 283 & 50.94 & 53.8 & 5 \\
\hline 0.20 & 283 & 56.6 & 53.8 & 5 \\
\hline 0.17 & 283 & 48.11 & 53.8 & 10 \\
\hline
\end{tabular}

Table 5. Theoretical predictions for sample 1 in Table 4 using even samples in Table3. The average prediction is equal to 53.77 hours with $3 \%$ error with experimental results.

\begin{tabular}{|l|l|l|l|l|}
\hline$\tau / R_{0}(\mathrm{hrs} / \mu \mathrm{m})$ & \multicolumn{1}{|c|}{$R_{10}(\mu \mathrm{m})$} & $\begin{array}{l}\tau_{\text {uneven }}(\mathrm{hrs}) \\
\text { Prediction }\end{array}$ & $\begin{array}{l}\tau_{\text {uneven }}(\mathrm{hrs}) \\
\text { Experiment }\end{array}$ & \% Error \\
\hline 0.21 & 286 & 60.06 & 48.28 & 24 \\
\hline 0.18 & 286 & 51.48 & 48.28 & 6 \\
\hline 0.20 & 286 & 57.2 & 48.28 & 18 \\
\hline 0.17 & 286 & 48.62 & 48.28 & 0.7 \\
\hline
\end{tabular}

Table 6. Theoretical predictions for sample 2 in table 4 using even samples in Table3. The average prediction is equal to 54.34 hours with $12 \%$ error with experimental results. 


\begin{tabular}{|l|l|l|l|l|}
\hline $\begin{array}{l}\tau / R_{0}(\mathrm{hrs} / \mu \mathrm{m}) \\
\text { Even sample }\end{array}$ & \multicolumn{1}{|c|}{$R_{10}(\mu \mathrm{m})$} & $\begin{array}{l}\left.\tau_{\text {uneven }}(\mathrm{hrs})\right) \\
\text { Prediction }\end{array}$ & $\begin{array}{l}\tau_{\text {uneven }}(\mathrm{hrs}) \\
\text { Experiment }\end{array}$ & Error \\
\hline 0.21 & 273 & 57.33 & 57.07 & 0.4 \\
\hline 0.18 & 273 & 49.14 & 57.07 & 13 \\
\hline 0.20 & 273 & 54.6 & 57.07 & 4 \\
\hline 0.17 & 273 & 46.41 & 57.07 & 18 \\
\hline
\end{tabular}

Table 7. Theoretical predictions for sample 3 in table 4 using even samples in Table3. The average prediction is equal to 51.87 hours with $10 \%$ error with experimental results.

\section{Experimental calibration for CPD}

The experimental results shown in this thesis were used to calibrate the CPD simulations conducted by the Kosztin group. To predict the fusion of multicellular systems, while the volume is not conserved, one can employ the CPD simulation for the conserved case and calibrate it with experimental results to predict the shape evolution process for multicellular systems (see (McCune et al. 2014) for more details).

By considering the Experiments and Simulations for equal size and unequal size as $\mathrm{E}_{1}, \mathrm{E}_{2}, \mathrm{~S}_{1}$ and $\mathrm{S}_{2}$ respectively and with $a_{0}=0.7$ and $\lambda=0.02 h^{-1}$ the simulation can provide a quantitative prediction for the 
shape evolution of experimental aggregates that can be matched with $E_{1}$ (McCune et al. 2014).

The goal was to predict $E_{2}$ directly from $S_{2}$. To do so the CPD time unit $\mathfrak{J}_{2}$ in terms of $\mathfrak{I}_{1}$ must be determined. It was shown that $C_{a} \tau_{E_{1,2}} \approx \tau_{S_{1,2}} \Im_{1,2}$ and $\mathfrak{I}_{2} \equiv \mathfrak{I}_{2, \tau} \approx \mathfrak{J}_{1} \frac{\tau_{E_{2}}}{\tau_{E_{1}}} \frac{\tau_{S_{1}}}{\tau_{S_{2}}}$ (for details see (McCune et al. 2014)) assuming the materials in both experiments are the same (constant $\eta / \gamma$ ). The CPD time unit $\mathfrak{I}_{2}$ was found as:

$$
\mathfrak{I}_{2} \equiv \mathfrak{I}_{2, R} \approx \mathfrak{I}_{1} \frac{R_{10, E_{2}}}{R_{0, E_{1}}} \frac{R_{0, S_{1}}}{R_{10, S_{2}}}
$$

With the experimental data shown in this chapter the Kosztin group could obtain $\mathfrak{J}_{2, R}=2.7 \mathrm{~min}$ and $\mathfrak{I}_{2, \tau}=2.3 \mathrm{~min}$ (McCune et al. 2014) and with either of these two CPD time units one could successfully predict $E_{2}$ (McCune et al. 2014). Hence by using equation 4.7 one can predict $E_{2}$ using $S_{1}, S_{2}$ and with the aid of the calibrating experiment $E_{1}$, without the need for intermediate continuum theory. This means that by calibrating the CPD simulation, as long as we are working with the same material (constant $\eta / \gamma$ ), the experimental outcome with any geometry could be predicted (McCune et al. 2014). 


\section{Conclusions}

Fusions experiments were performed with both $\mathrm{CHO}$ cell aggregates and HSF aggregates prepared by the EHAM method. In the case of $\mathrm{CHO}$ aggregates fusion was studied for the early linear fusion regime as well for the full fusion process. The characteristic fusion time evaluated by using the corresponding linear and exponential fit showed about $20 \%$ difference.

Fusion experiments were also performed with equal and unequal size HSF aggregates. Based on these experiments the theory, originally formulated for volume conserving aggregates had to be modified to allow for variation in volume. The modified theory described well the experimental results, the discrepancy between theoretical predictions and the experimental results being about $10 \%$.

The experimental results described in this chapter were also used to calibrate and validate the CPD formalism (McCune et al. 2012). 


\section{Chapter V: Fusion of Cylindrical aggregates}

Tubular organ structures can be bioprinted using either spherical or cylindrical bioinks. To optimize the tissue engineering of tubular organ structures by the bioprinting process, one needs to compare the fusion of cylindrical bioinks with that of spherical ones. In this chapter we detail the fusion experiments performed with cylindrical aggregates. It is this nontrivial case that first allowed verifying both the theory and demonstrating the power of CPD in its use to optimize the bioprinting of cylindrical bioink units. In fact it is on the basis of these experiments that it became evident that the original formulation of the theory (Kosztin et al, 2012) required modifications due to the variation of the length and volume of the fusing cylinders (see also the discussion on the variation of the volume of spherical aggregates analyzed in Chapter IV).

The fusion experiments reported in this chapter were performed with HSF cells. In order to consistently compare cylindrical and spherical aggregate fusion both had to be prepared by the same

method. As the EHAM method (see Chapter IV for the fusion of spherical aggregates prepared by this method) does not allow making 
cylinders, we used the sausage method, as described in Chapter II, to prepare both the spherical and cylindrical aggregates.

Below we describe fusion of both spherical and cylindrical HSF aggregates prepared by the sausage method. As for the cylinders, these experiments represent the first detailed quantitative study of the fusion of cylindrical bioink particles. As will be evident these experiments were complex, highly labor intensive and time consuming.

The fusion process for cylinders was followed in two different manners. Firstly pairs of cylinders were printed in grooves inside agarose, and secondly flat surfaces of agarose were prepared and pairs of cylinders were printed on them to fuse. The later experiments needed more skills and practice to avoid any shaking in the fusing samples, which could cause experimental failure very easily.

\section{Fusion of spherical HSF aggregates}

For the fusion of spherical aggregates pairs with similar radii were selected by microscopic observation. Fusion experiments were performed using 24 -well culture plate $(1.5 \mathrm{~cm}$ diameter of each well). Each well was covered with 3 droplets of agarose to prevent the sticking of aggregates to the plastic. Once agarose solidified, wells were filled with medium and pairs of aggregates were placed into the 
wells contiguous with each other with the help of a hair loop (Figure 23). The fusion process was followed in time by recording snapshots at different time points with a digital camera (Nikon, Japan) attached to an Olympus IX70 (Center Valley, PA) inverted microscope (Figure 12).
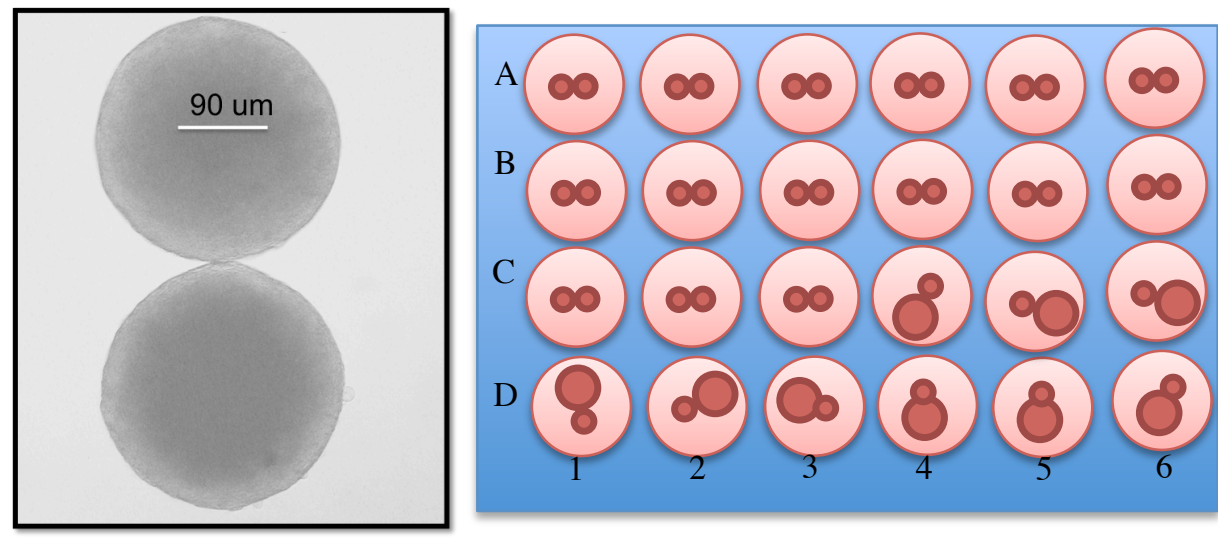

Figure 23. Left. Two similar size spherical aggregates prior to the initiation of fusion. Right. 24 well dish, each well covered with agarose containing pairs of similar size or different size aggregates in close proximity for fusion experiments.

\section{Analysis of the fusion of spherical aggregates}

The shape evolution of the fusion process was analyzed in terms of the recorded images using Mathematica (Wolfram, Mathematica 9, Champaign, IL). For each image, circular arcs were fitted to the fusing aggregates and the quantities $r, R$ and $\Theta$ (defined in Figure 10) were determined. Subsequently, $\sin (\Theta)$ was plotted versus time and the characteristic fusion time $\tau$ was evaluated. 


\section{Fusion of cylindrical HSF aggregates}

Fusion experiments with cylinders were performed in two different ways. In one case, cylinders were placed contiguously into grooves of semicircular cross section created in an agarose platform. In the other case cylinders were placed next to each other on flat agarose surfaces. For the recording of the fusion process, in both cases medium was removed, and samples were fixed under a chemical hood, using $4 \%$ paraformaldehyde (Alfa Aesar, Ward Hill, MA), for 30 min, followed by three rinses with PBS for 20 min. After fixation, fused pairs of cylinders were placed on $10 \mathrm{~cm}$ Petri dishes covered with $15 \mathrm{ml}$ solid agarose and were embedded in $15 \mathrm{ml}$ week agarose gel. The dishes were placed in a refrigerator until imaging (Figure 24). Longer cylinders sometimes slightly curled during the fusing process, but could easily be straightened. 

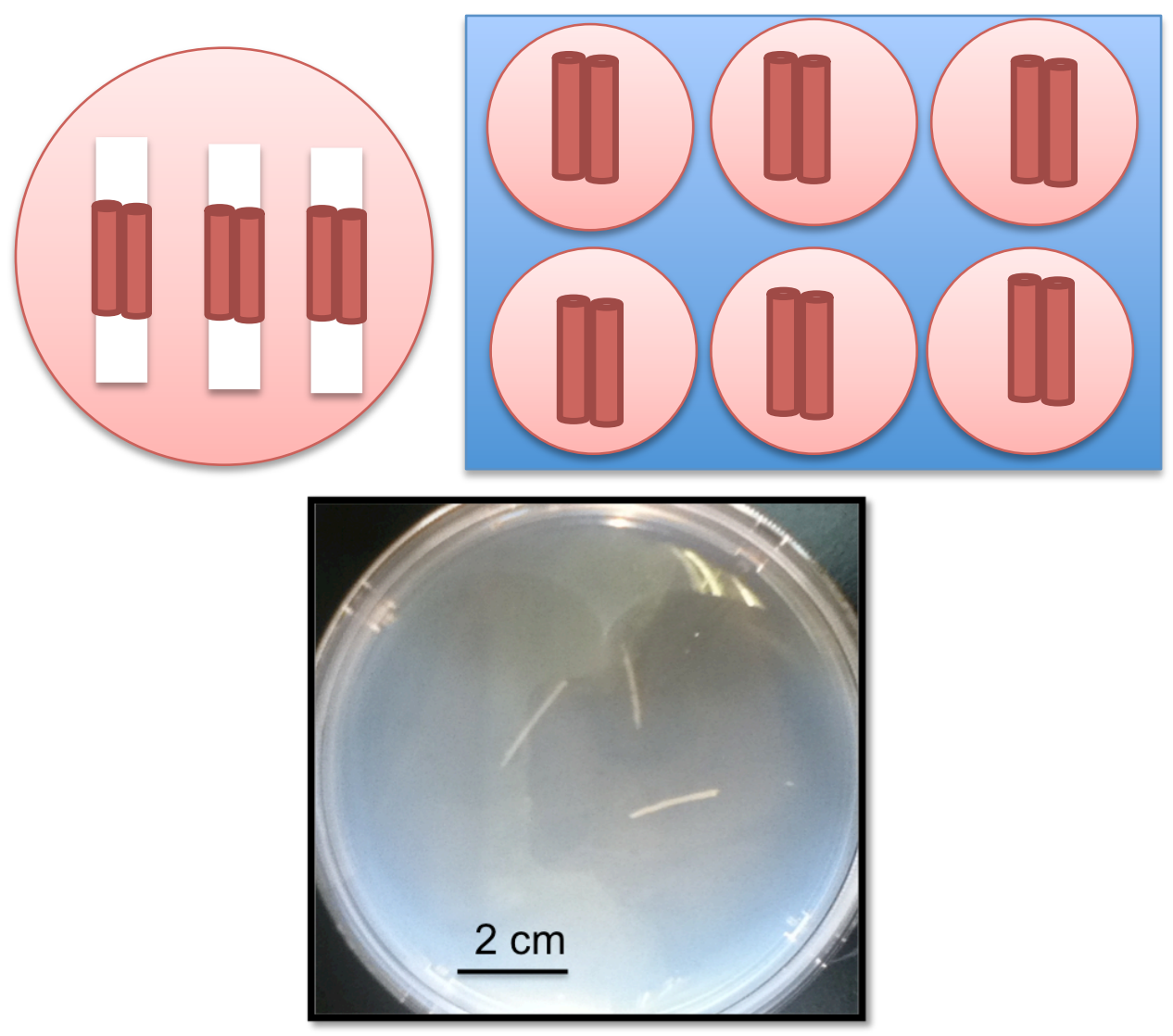

Figure 24. Upper Left. Pairs of fusing cylindrical aggregates in grooves. Upper Right. Pairs of fusing cylindrical aggregates in 6-well culture dish. Lower panel. Three pairs of fused cylinders embedded in agarose.

For imaging, the fused cylinders were cut into small pieces. The pieces were flipped by $90^{\circ}$ so as to lay with their cross section on a flat surface (see Figure 25). The contours of the fused cross sections were recorded by taking photographs with a digital camera (Nikon, Japan) attached to an Olympus IX70 (Center Valley, PA) inverted microscope. 


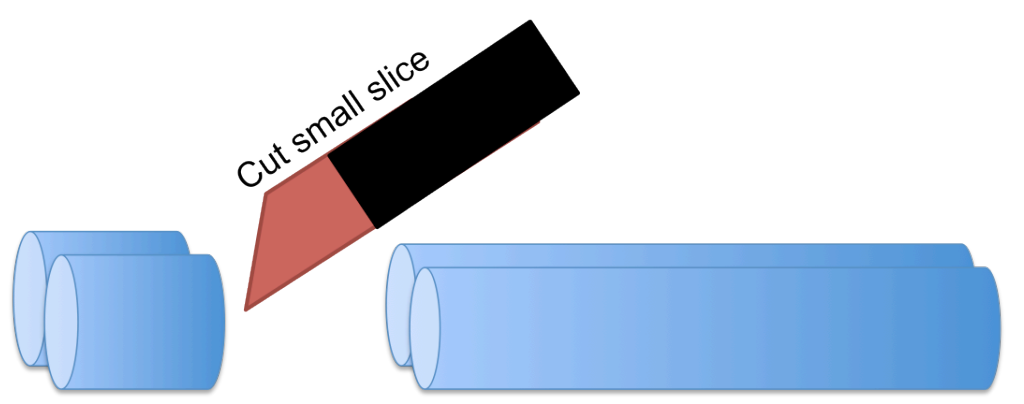

Flip 90 degree

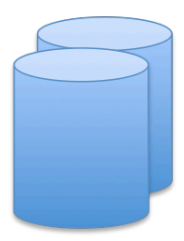

Ready for imaging under the microscope

Figure 25. Sample preparation for imaging. Cylindrical aggregates were cut in small pieces and were flipped to allow for the cross sectional images under the microscope.

Note the difference between the recording of the fusion process for spherical and cylindrical aggregates. For spherical aggregates the photographs for different time points could be taken on the same pair (pairs were kept in the incubator between successive time points). For cylinders, however, the samples on which images were taken at any given time point had to be discarded. Thus, for cylinders a large number of pairs were prepared at $t=0$. At the first time point, two hours, three pairs of fusing aggregates were fixed (the others kept in the incubator and let to continue fusing) and then sectioned into the small cylinders shown in Figure 25. These were imaged and then discarded. At the later time points another set of fusing aggregates 
were selected from the initially prepared ones, fixed, sectioned, imaged and discarded. This process was continued until images were prepared at all the desired time points.

Thus, contrary to spherical aggregates, for cylinders, at each time point different pairs of fused aggregates had to be imaged. Even though the initial pairs were prepared similarly, they were clearly not identical. To mitigate the unavoidable error stemming from the difference in the fusing cylinders, at each time point a number of sections were imaged and analyzed and the results eventually averaged. Specifically, the cross section of the small cylinder pairs (see Figure 25) was fitted with two circular arcs (similarly to the spherical aggregates) and the quantities $R$ and $\Theta$ (defined in Fig. 10) for the samples that belonged to the same time point were averaged. These averages were used to calculate $\sin (\Theta)$, which then was plotted versus time and the characteristic fusion time $\tau$ was determined again for cylinders (for details see (Kosztin et al. 2012)).

\section{Cylinder fusion in grooves}

In this case, after preparation the cylindrical aggregates were printed into grooves prepared inside an agarose layer. For this $10 \mathrm{~cm}$ Petri 
dishes were filled with liquid agarose and $1.35 \mathrm{~mm}$ outer diameter glass tubes were embedded in the liquid. Grooves were made by removing the glass tubes after agarose solidification. Pairs of cylinders were placed inside these grooves and their fusion recorded at 2, 4, 7 , $11,16,22,29$ hours after the starting moment of fusion, as described above. Figure 26 shows the grooves with cylinders inside them.
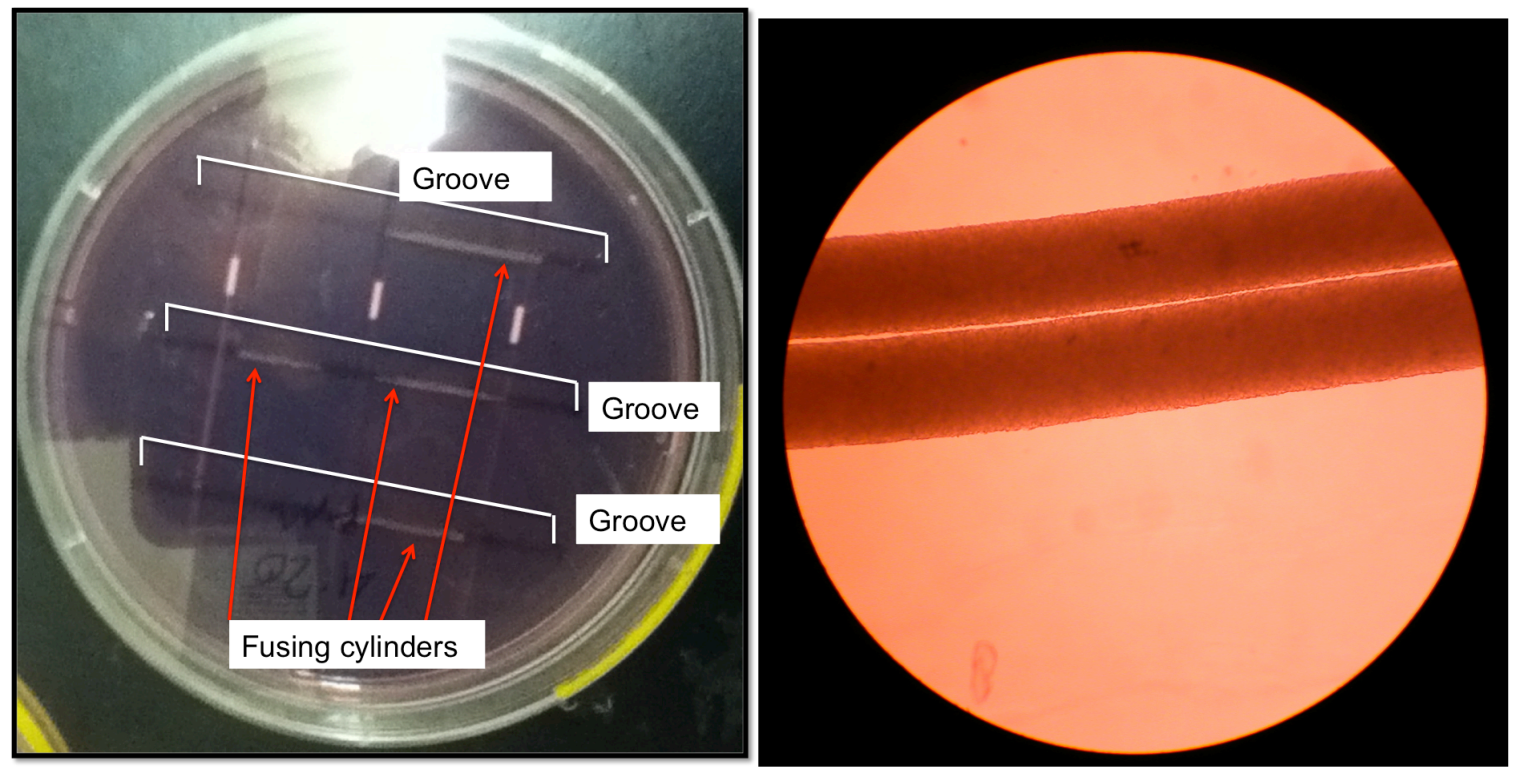

Figure 26. Left. Cylindrical aggregates (white) inside agarose grooves. Right. Two fusing cylinders at $\mathrm{t}=0$ under microscope.

For each time point images of several samples were analyzed, as detailed above, and the average represented as $\sin \theta$ for that time point. 


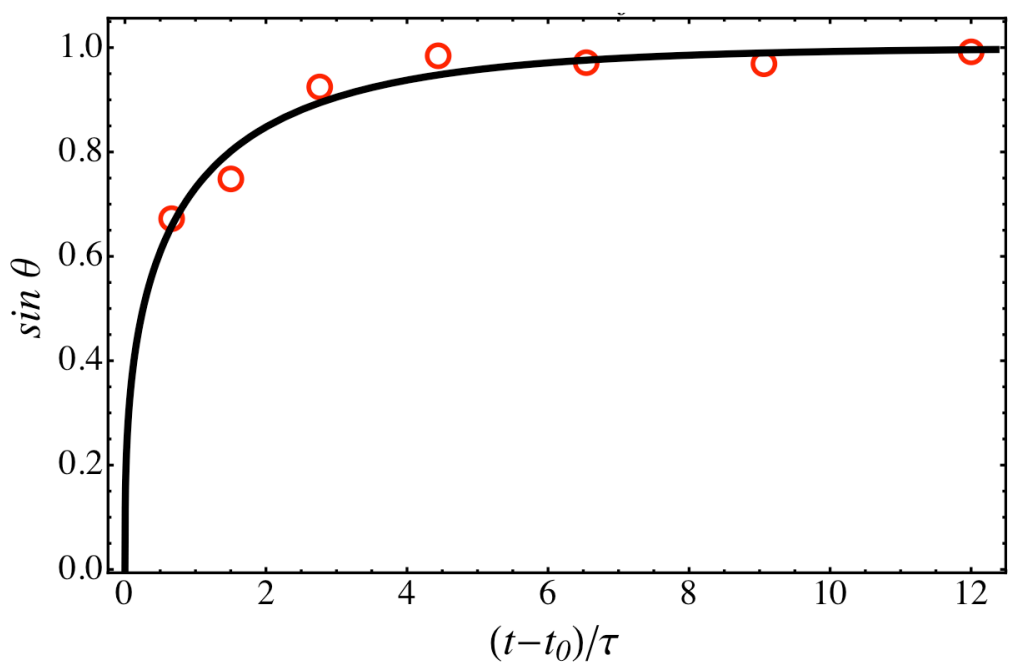

Figure 27. $\sin \theta v \boldsymbol{s} .\left(t-t_{0}\right) / \tau$ for fusing cylinders inside grooves.

Figure 27 shows the $\sin \theta$ vs. $\left(t-t_{0}\right) / \tau$ for fusing cylinders in grooves. The characteristic fusion time, $\tau$ for the particular experiment shown in Figure 27, was 3.06 hours. Considering the time scale of typical cellular motion (with typical cellular diffusion constant $\sim 10^{-12} \mathrm{~cm}^{2} / \mathrm{sec}$; (Beysens et al. 2000)), this number is spurious, implying an unrealistically fast fusion process. Upon further analysis we realized that fusion of cellular cylinders in grooves is a process that takes place under the influence of an external force: the fusing aggregates, due to gravity, press against each other (note that the bottom of the groove, as a consequence of its making, is not flat). Thus the above fusion time reflects not the genuine coalescence of the two cylinders but involves also the distortion of the fusion contour due the external 
force. As the effect of their mutual pressing, in essence, the cylinders appear to be partially fused already at $\mathrm{t}=0$.

To extract the characteristic fusion time due exclusively to the coalescence of the two cylinders, as a result of their apparent liquid properties, the fusion experiments were also performed on flat surface.

\section{Cylinder fusion on flat agarose surface}

6-well plate culture dishes $(3.5 \mathrm{~cm}$ diameter of each well) were used for fusion of cylindrical aggregates without groove. Each well was covered with $3 \mathrm{ml}$ of agarose and after it solidified, each well was filled with medium. Pairs of cellular cylinders with length of $2.5 \mathrm{~cm}$ were put in each well and were placed contiguously side-by-side prior to the fusion. For each data point at 2, 6, 10, 12, 22, 24, 36 and 48 hours after the starting of, one 6 well dish with 4-6 pairs of fusing cylinders was used. Later we compare the results of fusion of cylinders on flat agarose with spherical aggregates on flat agarose. 


\section{Spheroid Fusion}

In this experiment the fusion process of 4 pairs of HSF spherical aggregates was tracked. Figure 28 shows one of these fusion sequences.

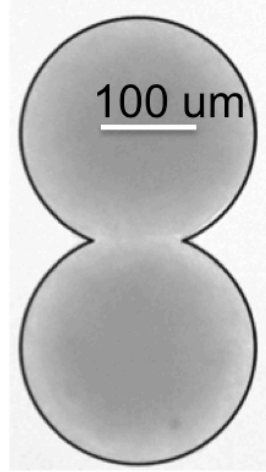

$\mathrm{t}=10 \mathrm{~h}$

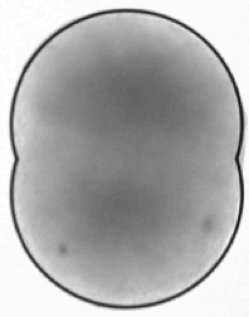

$\mathrm{t}=62 \mathrm{~h}$

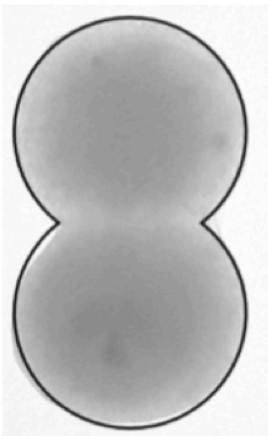

$\mathrm{t}=18 \mathrm{~h}$

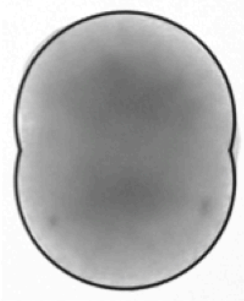

$\mathrm{t}=83 \mathrm{~h}$

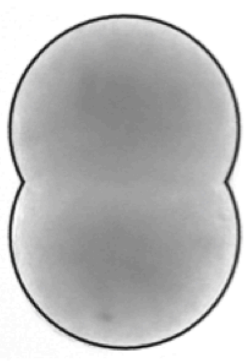

$\mathrm{t}=33 \mathrm{~h}$

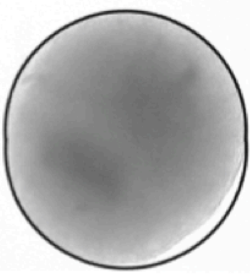

$\mathrm{t}=107 \mathrm{~h}$

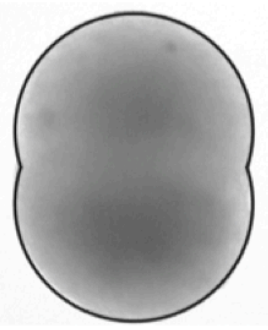

$\mathrm{t}=47 \mathrm{~h}$

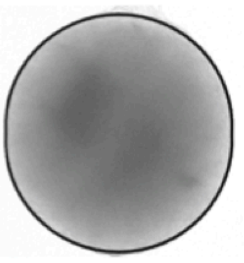

$\mathrm{t}=141 \mathrm{~h}$

Figure 28. A fusion sequence for spherical HSF aggregates prepared by the sausage method.

As found in Chapter IV, the volume of HSF spheroids, prepared with 
the EHAM method decreases during fusion (McCune et al. 2014). We found this to be the case also for HSF spheroids prepared with the sausage method, albeit with slower decrease. Figure 29 shows the $\sin ^{2} \theta$ vs. $\left(t-t_{0}\right) / \tau$ and the volume decrease for one pair of HSF spheroids prepared by the sausage method. Table 8 lists all the fusion parameters introduced earlier, found for the 4 fusing HSF spheroid pairs.
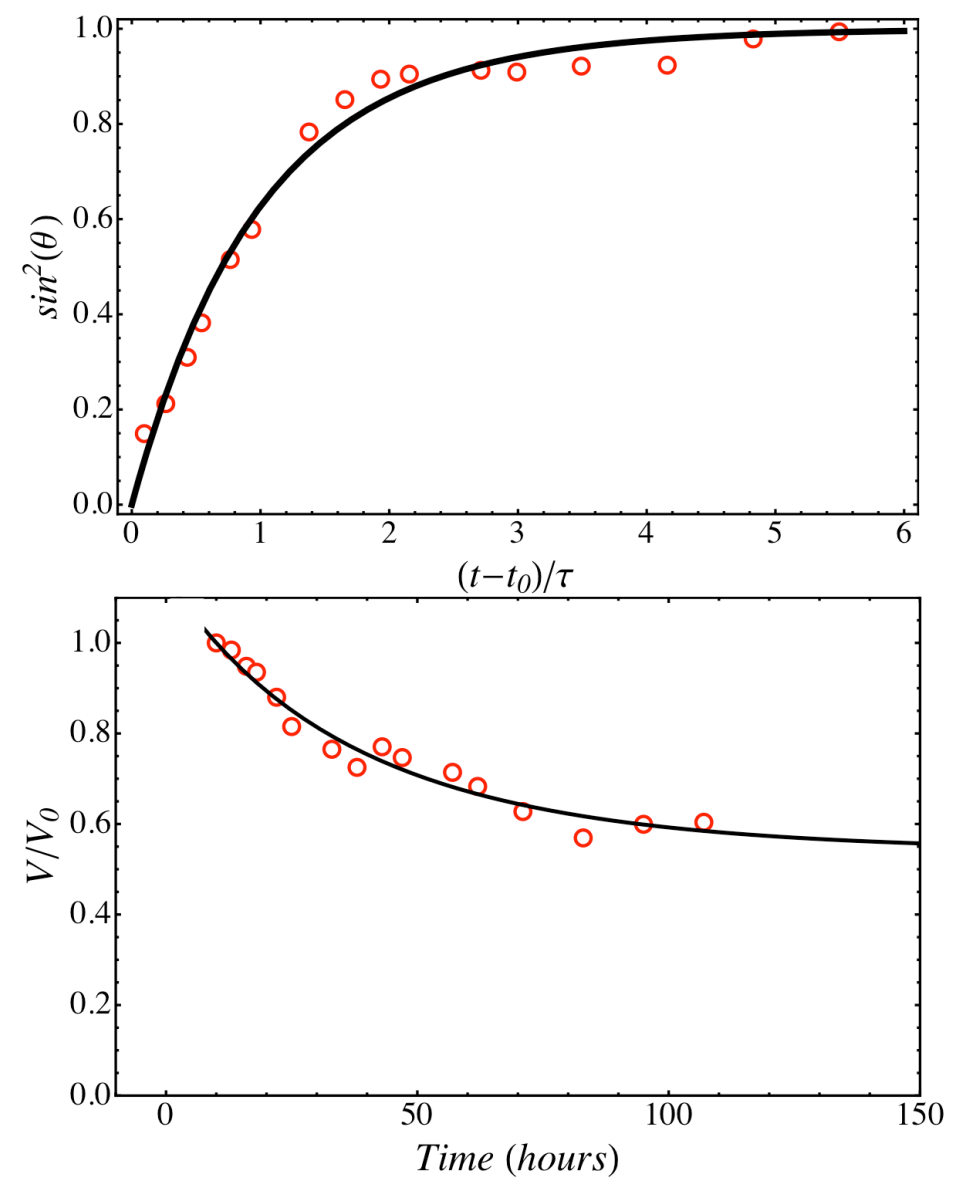

Figure 29. $\sin ^{2} \theta$ vs. $\left(\boldsymbol{t}-\boldsymbol{t}_{\mathbf{0}}\right) / \boldsymbol{\tau}$ (upper panel) and volume decrease (lower panel) for fusing HSF spheroids. 
This outcome shows the effect of aggregate preparation on the material properties of aggregates. The fact that the surface tension of cellular aggregates prepared by the sausage method is higher than that of aggregates prepared by the EHAM method (compare 49.77 dyne/cm with 72.7 dyne/cm, the respective values for the surface tension as obtained in Chapter IV for HSF aggregates prepared with the EHAM method and by Norotte (Norotte 2009) for HSF aggregates prepared with the sausage method), implies aggregates prepared by the sausage method are more compact therefore their volume decreases slower during fusion. We will discuss these issues in more details in Chapter VI.

\begin{tabular}{|l|l|l|l|l|l|l|}
\hline Sample & $\tau_{\text {eff }}$ (hours) & $\mathrm{R}_{0}(\mu \mathrm{m})$ & $\tau / \mathrm{R}_{0}$ & $\mathrm{a}_{0}$ & $\lambda_{\mathrm{a}}$ & $\tau$ \\
\hline 1 & 18.2 & 229 & 0.0795 & 0.84 & 0.034 & 19.78 \\
\hline 2 & 22.0 & 220 & 0.100 & 0.74 & 0.022 & 25.28 \\
\hline 3 & 19.1 & 199 & 0.096 & 0.75 & 0.016 & 21.82 \\
\hline 4 & 25.1 & 216 & 0.116 & 0.78 & 0.021 & 28.20 \\
\hline
\end{tabular}

Table 8. Results for fusion of HSF aggregates prepared by sausage method 
The average characteristic fusion time, as obtained from the results shown in Table 8 is 23.77 hours with standard deviation equal to 3.22 hours.

\section{Cylinder fusion}

In the case of HSF cylinders the fusion process was analyzed as explained earlier. Figure 30 shows snapshots for 2, 6, 10, 22, 36 and 48 hours for one fusion sequence (i.e. using just one sample at each time point). The plot of $\sin \theta$ versus time shown in Figure 31 was prepared by averaging the results over 8 samples (i.e. images) at each time point. 


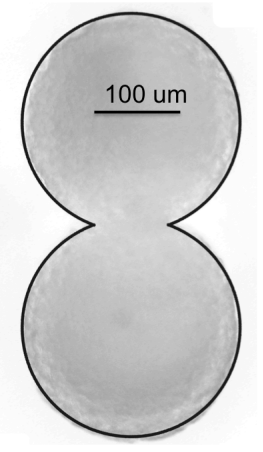

$t=2 h$

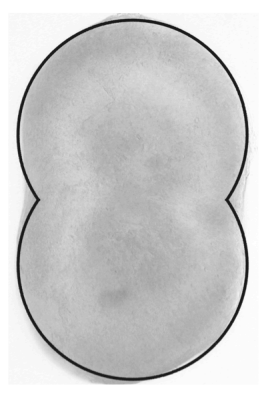

$\mathrm{t}=22 \mathrm{~h}$
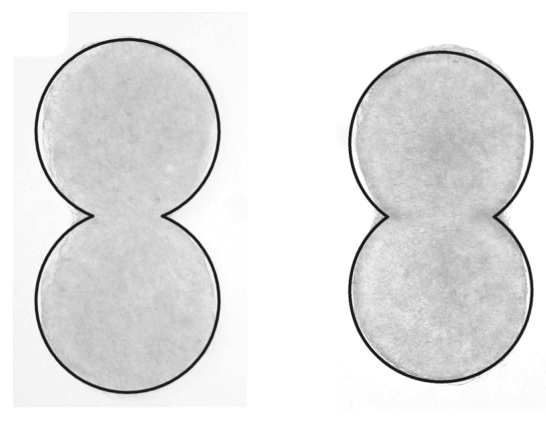

$\mathrm{t}=6 \mathrm{~h}$

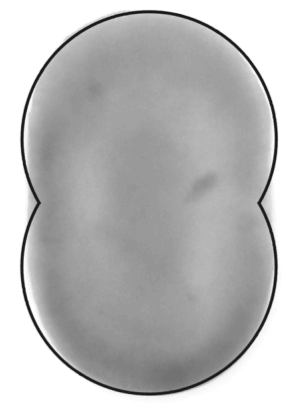

$\mathrm{t}=36 \mathrm{~h}$

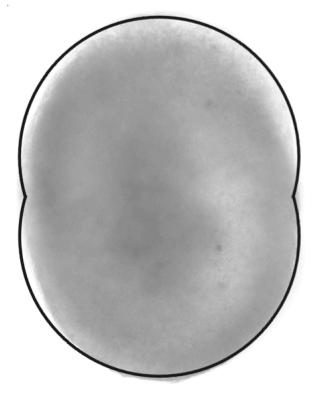

$\mathrm{t}=48 \mathrm{~h}$

Figure 30. Cross sectional images of fusing cylinders at selected time points.

As could be predicted, the spread for cylindrical aggregates in Figure 31 is larger compared to that for spheroids in the Figure 29 (i.e. same spherical sample in Figure 29 versus average over 8 different cylindrical samples in Figure 31). Despite the larger spread, the data in both figures can be well fitted with theory (for details see (Kosztin et al. 2012)). 


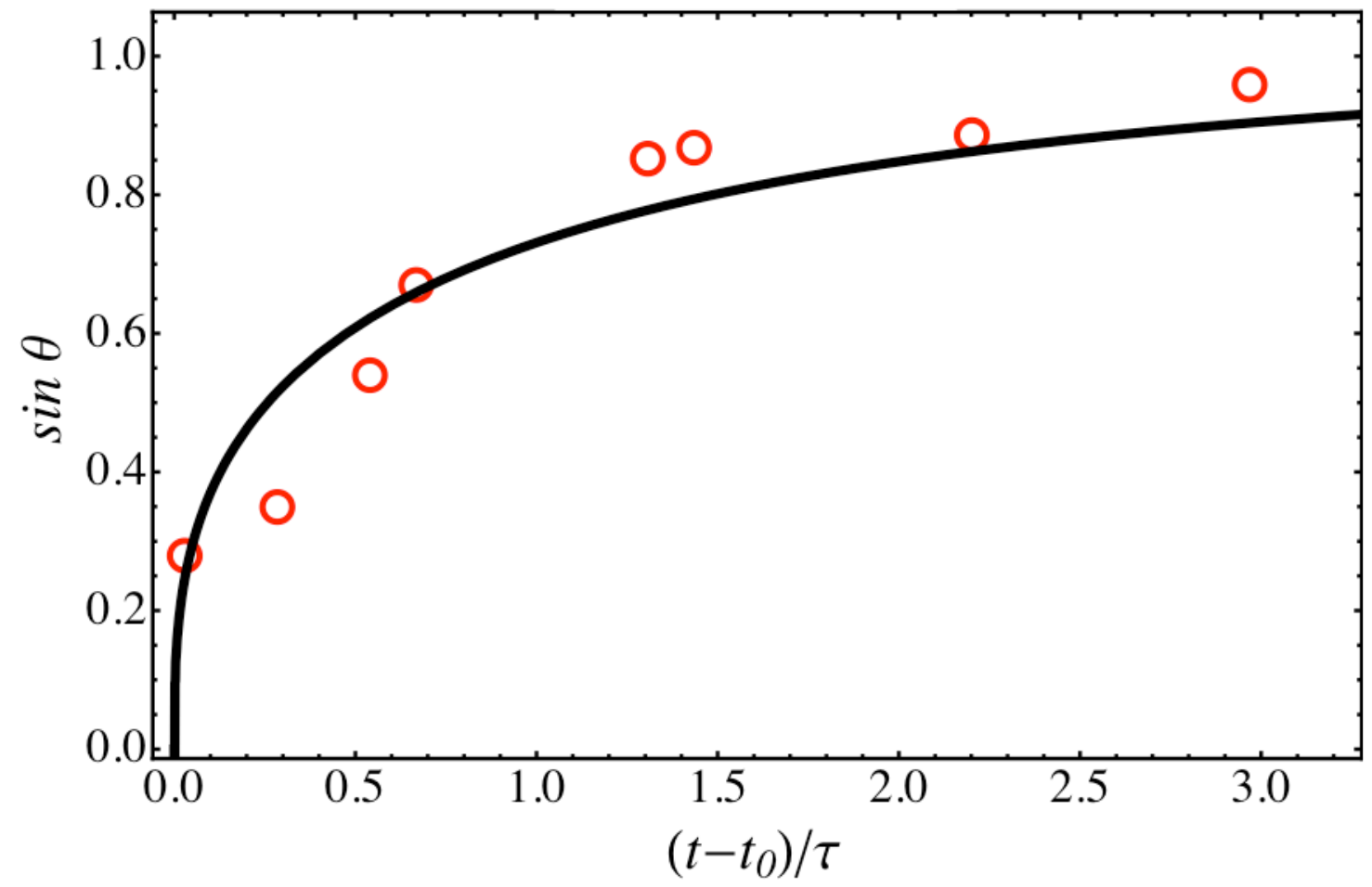

Figure 31. $\sin \theta \boldsymbol{v s} .\left(\boldsymbol{t}-\boldsymbol{t}_{\mathbf{0}}\right) / \boldsymbol{\tau}$ for cylindrical HSF aggregates prepared with the sausage method. Red circles represent the average of $\sin \theta$ over 8 samples at each time point (except for the first data point for which see text). The solid line is the theoretical fit to the data.

The characteristic fusion time $\tau$ obtained from the graph in Figure 31 is 15.65 hours, with the fitting parameters $t_{0}$ of 1.54 hours. As it was introduced in Chapter IV, $t_{0}$ is a fitting parameter for the exponential fit, which gives the de facto time when fusion has started. 
The first data point in Figure 31 corresponds to observations at $2 \mathrm{~h}$ after the initiation of fusion. Collecting data at this early time could not be done consistently (the data point in Figure 31 corresponds to a few selected images at $2 h$, whereas all the other points represent averages over 8 samples. Even if some samples could be photographed (see Figure 30), some samples were very hard to handle, fix and image at $2 \mathrm{~h}$, as in those samples aggregates showed poor fusion due to the loose connection between them at this early time point. With this reasoning we ignored the data point for 2 hours and re-plot the $\sin \theta v s .\left(t-t_{0}\right) / \tau$ in Figure 32. The exponential fit to these data resulted in a characteristic fusion time equal to 9.95 hours (with $\mathrm{t}_{0}=5.23 \mathrm{~h}$ ). 


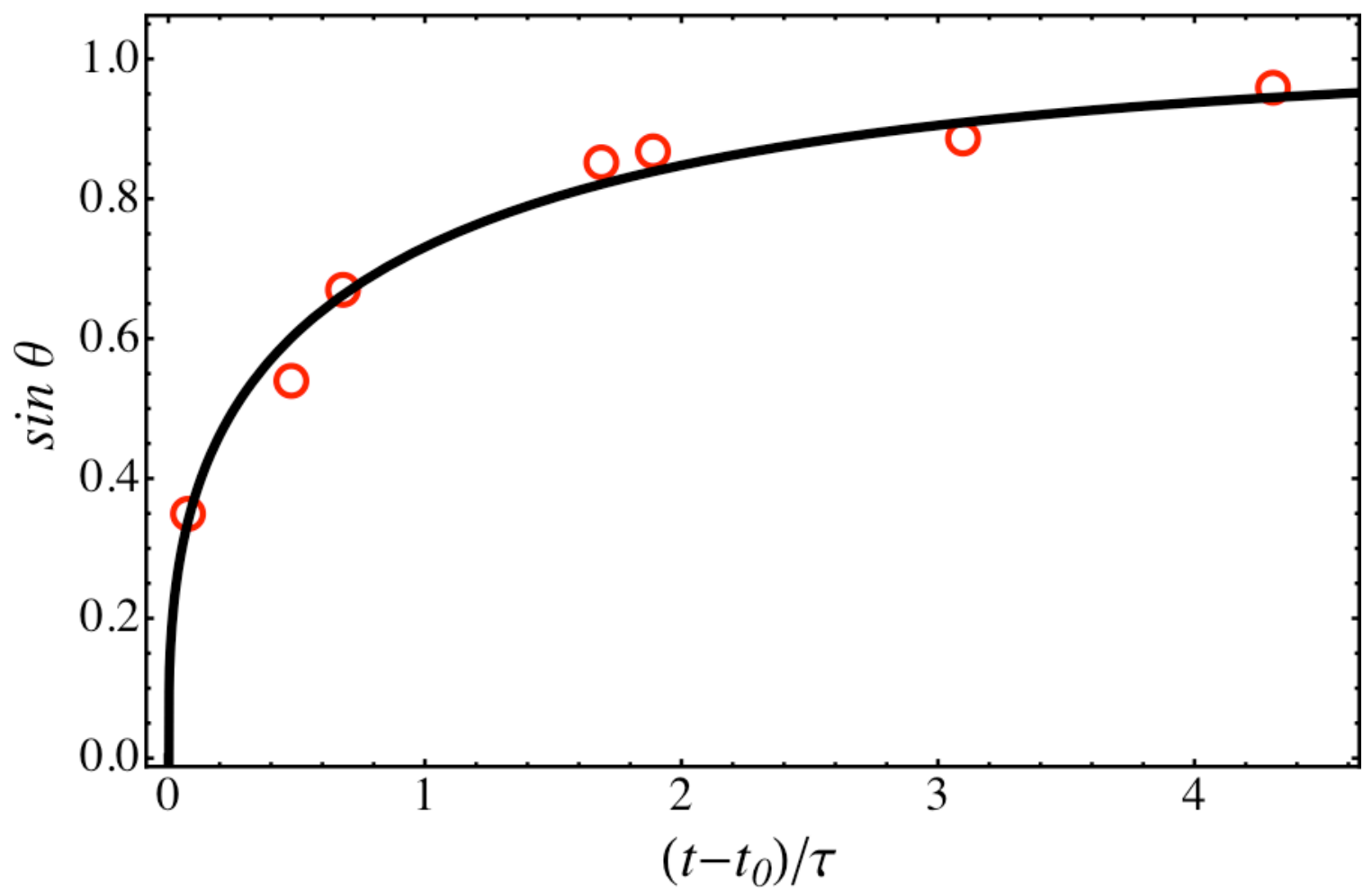

Figure 32. $\sin \theta \boldsymbol{v s} .\left(\boldsymbol{t}-\boldsymbol{t}_{\mathbf{0}}\right) / \boldsymbol{\tau}$ for cylindrical HSF aggregates prepared with the sausage method. Open circles represent the average of $\sin (\Theta)$ over 8 samples at each time point

Just as in the case of fusing spheroids, we found that the geometric properties of the fusing cylindrical aggregates also varied in time. As shown in Figure 33 the length of fusing cylinders contract during the fusion process and this decrease can be well fitted with an equation similar to that for the radii of fusing spheroids (see Eq. 4.3)

$$
L / L_{0}=b(t)=b_{0}+\left(1-b_{0}\right) \exp \left(-\lambda_{b} t\right)
$$




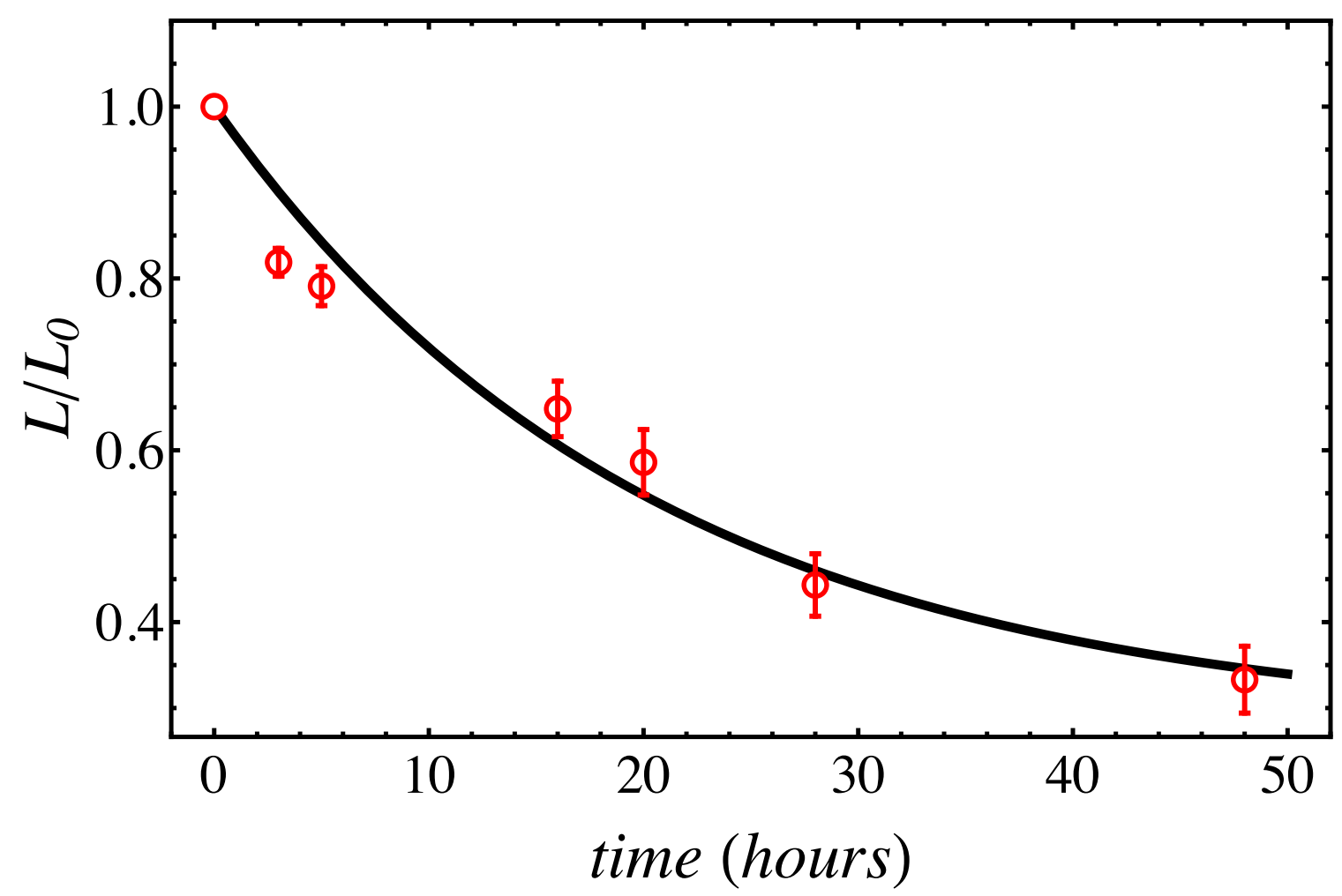

Figure 33. Length contraction of fusing cylinders. Open circles represent data averaged over 4 pairs of cylinders with the corresponding error bars. The solid line is the fit to Eq. (5.1) with $b_{0}$ and $\lambda_{b}$ equal to 0.28 and 0.05 respectively.

This change in length again leads to correction in the characteristic fusion time as we had in Chapter IV. To find the combination $\frac{\eta R_{0}}{\gamma}$ that is the actual $\tau$, one needs to divide $\tau_{e f f}$, the measured characteristic fusion time, by a constant to get a closer fit to the theoretical result. In this case one could easily find the value 0.8 to relate the $\tau_{\text {eff }}$ to the $\tau$. Correspondingly the final $\tau$ for two fits are 12.43 and 19.56 . 


\section{Conclusion}

Spherical and cylindrical HSF aggregates were prepared with the same method, namely the sausage method. Fusion experiments were performed with both spherical and cylindrical aggregates, in the latter case using two approaches. Cylinder fusion experiments and their evaluation were challenging. First, when such experiments were carried out in grooves of semicircular cross section the outcome was spurious due to the force exerted by the fusing aggregates on each other. Thus fusion was also investigated on flat surfaces. Second, the fusion sequence was not possible to follow on the same pair of cylindrical aggregates as aggregates had to be discarded after each recording (this unavoidably leads to larger errors in the values of the fusion parameters than in the case of spheroids). Third, due to the previous constraint, large numbers of aggregates needed to be prepared making the experimental work highly time consuming. Fourth, recording of the fusion of the aggregates had to be carried out with extreme care (e.g. shaking of the culture dish containing the fusing pair needed to be avoided while carried from the incubator to the microscope stage to not shake the fusing pair and thus distort the geometry). Despite the above difficulties and constraints, in both cases theory provided a good fit to the experimental data. In particular it showed that the characteristic fusion time for spheroids ( $\sim 23 \mathrm{~h})$ is 
larger than that for cylinders $(\sim 12.5 \mathrm{~h})$. This suggests that building $3 \mathrm{D}$ biological constructs with tubular bioink particles (in particular by bioprinting) may lead to a final product faster. Note that spherical aggregates require 48 hours to round up. By then the cylindrical aggregates already fused. 


\section{Chapter VI: Effect of preparation on the biomechanical properties of bioink units}

Preparing the bioink is one of the most important parts of bioprinting. To select the appropriate method and make the suitable bioink for each application, one needs to compare the aggregates prepared with different methods. In this chapter we compare the difference in the properties of the multicellular bioink units (i.e. cylindrical and spherical aggregates), such as surface tension and characteristic fusion time, prepared by the EHAM and sausage method, that were described in Chapter II. These differences will need to be taken into consideration when building tubular constructs by the bioprinting technology.

\section{Apparent tissue surface tension (ATST)}

In the case of $\mathrm{CHO}$ cells, for the ATST of aggregates prepared by the EHAM and sausage method, the respective values were found to be 14.71 dyne/cm as measured by us (see Chapter III) and 22.8 dyne/cm, as reported by Norotte and co-workers (Norotte et al. 2008). We note that the same cells were used in both cases. 
Similarly large differences were found in the case of HSF aggregates prepared by the two methods. The respective values for the EHAM and sausage method were 49.77 dyne/cm, as reported in Chapter III and 72.7 dyne/cm, as found by Norotte and co-workers (Norotte 2009).

\section{Characteristic fusion time}

To understand the effect of aggregate preparation on the characteristic fusion time, we compared the fusion for 8 pairs of $\mathrm{CHO}$ aggregates prepared with EHAM, described in Chapter IV, with 6 pairs of $\mathrm{CHO}$ aggregates prepared with sausage method.

$\mathrm{CHO}$ aggregates were prepared with sausage method that was described in Chapter II and the fusion process, as detailed in Chapter IV and $V$, was followed by recording the shape of the aggregate pairs at time $0,2,5,8,11,14,17,25,29,33,37,39,48,54,61,73,85$, $97,109,121,133,145,157$ and 173 hours. Images were analyzed and the characteristic fusion times for 6 pairs of $\mathrm{CHO}$ aggregates prepared with sausage method were determined (Figure 34).

As demonstrated in Figure 34, red squares represent the characteristic fusion time for $\mathrm{CHO}$ aggregates prepared by EHAM method (duplicated from Figure 15) and blue diamonds show the 
characteristic fusion time for $\mathrm{CHO}$ aggregates prepared by the sausage method. As shown in Figure 34, results for the characteristic fusion time are quite consistent within the same preparation method but differed between the methods.

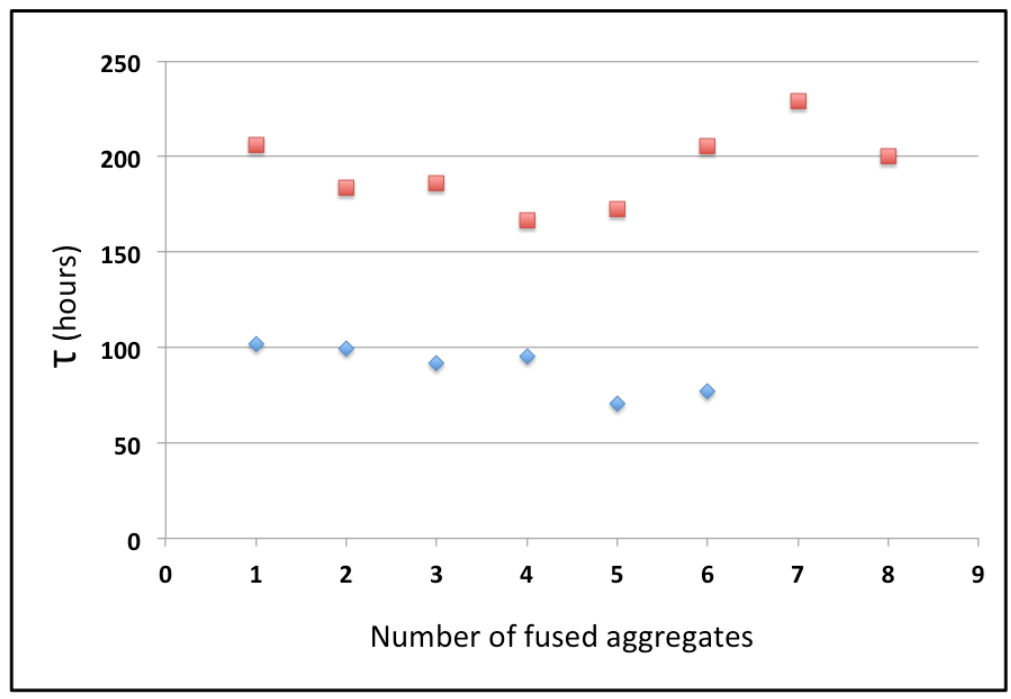

Figure 34. Comparison of the characteristic fusion time for $\mathrm{CHO}$ aggregates prepared by different methods. The red squares and the blue diamonds represent fusion times obtained respectively for aggregates prepared by the EHAM and sausage method.

The average characteristic fusion time over the 8 experiments with the EHAM method-prepared aggregates, as obtained from the exponential fit to all the data (see Figure 15 in Chapter IV) is $193.23 \pm 36$ hrs. The analogous result for the sausage method-prepared aggregates (blue diamonds in Figure 34 ) is $89.37 \pm 19 \mathrm{hrs}$. 
Fusion experiments with aggregates composed of HSF cells were descrbed in Chapters IV and V. The carachteristic fusion time found for the same size aggregates prepared by EHAM in Chapter IV was 46.73 hours while it was 23.77 hours for aggregates prepared by the suasage method described in Chpater V.

In both cases of HSF and $\mathrm{CHO}$ aggregates, the characteristic fusion time and ATST are different depending on aggregate preparation method. ATST of aggregates prepared by sausage method (HSF and $\mathrm{CHO}$ ) is higher than that of aggregates prepared by EHAM. This means the higher adhesion between the cells in the aggregates prepared with the sausage method. One can predict faster fusion based on the larger ATST, which is also observed in the experiments. Characteristic fusion time for aggregates prepared by sausage method is always less than that of aggregates prepared by EHAM and that means faster fusion. This is true if either the viscosity is constant or it is decreasing. Table 9 summarizes the characteristic fusion time, ATST and $\mathrm{R}_{0}$ for HSF aggregates prepared by EHAM and the sausage method from Tables 3 and 8 . Viscosity is calculated using these values and shows very small change (decrease) in the case of the sausage method (denoted by SM in Table 9). This is consistent with the fact that higher ATST implies faster fusion. 


\begin{tabular}{|c|c|c|c|c|}
\hline HSF & $\tau$ (hours) & $\begin{array}{l}\gamma \\
\text { (dyne/cm) }\end{array}$ & $R_{0}(\mathrm{~cm})$ & $\eta\left({ }^{\text {dyne. } s e c} / \mathrm{cm}^{2}\right)$ \\
\hline EHAM & 49.7 & 49.77 & 0.0228 & $3.9 \times 10^{8}$ \\
\hline EHAM & 42.66 & 49.77 & 0.0230 & $3.3 \times 10^{8}$ \\
\hline EHAM & 46.32 & 49.77 & 0.0231 & $3.6 \times 10^{8}$ \\
\hline EHAM & 48.25 & 49.77 & 0.0276 & $3.1 \times 10^{8}$ \\
\hline Average EHAM & 46.73 & 49.77 & 0.0241 & $3.47 \times 10^{8}$ \\
\hline SM & 19.78 & 72.7 & 0.0229 & $2.2 \times 10^{8}$ \\
\hline SM & 25.28 & 72.7 & 0.0220 & $3 \times 10^{8}$ \\
\hline SM & 21.82 & 72.7 & 0.0199 & $2.8 \times 10^{8}$ \\
\hline SM & 28.2 & 72.7 & 0.0216 & $3.4 \times 10^{8}$ \\
\hline Average SM & 23.77 & 72.7 & 0.0216 & $2.85 \times 10^{8}$ \\
\hline
\end{tabular}

Table 9. Comparison of data obtained with HSF aggregates prepared by EHAM and SM. While ATST is almost $50 \%$ bigger for SM the viscosity is also $17 \%$ smaller than $\left(3.9 \times 10^{8}\right)$ that for EHAM. Hence one can state that larger ATST causes faster fusion. 


\begin{tabular}{|l|c|c|c|l|}
\hline Cell & $\frac{\tau_{S M}}{\tau_{E H A M}}$ & $\frac{\gamma_{S M}}{\gamma_{E H A M}}$ & $\frac{R_{0 E H A M}}{R_{0 S M}}$ & \multicolumn{1}{|c|}{$\frac{\Delta \eta}{\eta_{E H A M}}$} \\
\hline CHO & $\frac{89.37}{193.23}=0.46$ & $\frac{22.8}{14.71}=1.54$ & $\frac{260}{230}=1.13$ & $19 \%$ \\
\hline HSF & $\frac{23.77}{46.7}=0.50$ & $\frac{72.7}{49.77}=1.46$ & $\frac{241}{216}=1.11$ & $17 \%$ \\
\hline
\end{tabular}

Table 10. Effect of aggregate preparation on the viscoelastic properties of multicellular systems composed of HSF and $\mathrm{CHO}$ cells.

It is clear that different aggregate preparation methods provide different surface tensions of cell aggregates. This causes change in viscosity. While ATST is larger for SM aggregates than EHAM aggregates the viscosity trend is the opposite. The viscosities obtained here are different from those in Chapter 3. The one represented there and extracted from compression experiments is a viscosity in the presence of external force (i.e. compressive force), whereas the viscosities shown in this chapter are not related to any force. The liquid viscosity should not depend on any force (the shearing force), but here the fact that the cells are not point particles, but have definite shape and that 
shape also relaxes (not only the aggregate as a whole) leads to the two viscosities that are not the same.

With these findings, we suggest to use bioink prepared with the method that provides the larges, as it makes post-printing fusion and thus structure formation faster. In terms of the equation $\tau=\eta R_{0} / \gamma$ one can conclude that larger ATST and lower lower viscosity lead to smaller $\tau$ which implies faster fusion.

\section{Conclusions}

The above results indicate that the biomechanical properties of bioink units strongly depend on the method of their preparation, as evaluated by the measurement of ATST and the characteristic fusion time.

The findings for the ATST imply that bioink units in the shape of multicellular spheroids are more adhesive when prepared by the sausage method than by the EHAM method. This may not be surprising, considering that the sausage method involves a centrifugation step to arrive at a compact cell pellet, which is aspirated into a micropipette and than extruded and cut into small cylinders with aspect ratio close to one. In the case of the EHAM method, no such compactification is used, as cells are allowed to assemble into 
spheroids without any external force (other than possibly the small gravitational force).

The results for the characteristic fusion time, $\tau$ are consistent with the above finding. According to Eq. 4.2, $\tau$ is inversely proportional to ATST, thus it should be smaller if ATST is larger (provided that viscosity is constant or decreasing). This is indeed the case, as for both the $\mathrm{CHO}$ and HSF aggregates $\tau$ was found larger in the case of aggregates prepared with the EHAM than with the sausage method. 


\section{CHAPTER VII: final conclusions and future work}

In this thesis we discussed the experimental component of the Cellular Particle Dynamics (CPD), a theoretical, computational, experimental framework that predicts the shape evolution of multicellular systems. The experimental results of this thesis work were used to verify parts of the theoretical component (i.e. predictions), as well as to calibrate the CPD parameters, the quantities playing crucial role in the computation component of the formalism. The specific novel accomplishments of this thesis work can be summarized as follows.

1. The EHAM method was used for the first time to prepare spherical aggregates for the measurement of ATST and their fusion.

2. Quantitative fusion experiments were performed with unequal size spherical aggregates (and used to verify the theoretical component of CPD).

3. Quantitative fusion experiments were performed with cylindrical aggregates and used to verify theoretical predictions. 
4. Quantitative comparison of the biomechanical properties of spherical and cylindrical bioink units prepared with two different methods, the EHAM and the sausage method was carried out.

5. Quantitative study of the volume dependence of the fusing aggregates was performed and results were used for the modification of CPD.

So far CPD has been applied to optimize postprinting structure formation of tubular organoids (e.g. vascular graft). However CPD can be used, in principle, for any biomechanical process that involves shape evolution. Some exciting topics that would merit further work, but were not part of this thesis are

1. It would be interesting to investigate the correlation between the observed decrease in the overall volume of the aggregates and the behavior and movement of individual cells within the aggregates.

2. A particularly exciting possible future application of CPD could be the systematic study of tumor shape change in the course of malignant transformations. 
3. A further significant generalization of CPD would be the incorporation of purely biological processes into the formalism, for example to correlate the genetic modifications in the tumors with their changes in shape. 


\section{REFERENCES}

Armstrong, P.B., 1989. Cell sorting out: the self-assembly of tissues in vitro. Critical reviews in biochemistry and molecular biology, 24(2), pp.119-149.

Atala, A., 2009. Engineering organs. Current Opinion in Biotechnology, 20(5), pp.575-592.

Atala, A., 2012. Regenerative medicine strategies. Journal of Pediatric Surgery, 47(1), pp.17-28.

Atala, A. et al., 2006. Tissue-engineered autologous bladders for patients needing cystoplasty. The Lancet, 367(9518), pp.12411246.

Basu, J. \& Ludlow, J.W., 2012. Developmental engineering the kidney: Leveraging principles of morphogenesis for renal regeneration. Birth Defects Research Part C: Embryo Today: Reviews, 96(1), pp.30-38.

Bellehumeur, C.T., Kontopoulou, M. \& Vlachopoulos, J., 1998. The role of viscoelasticity in polymer sintering. Rheologica acta, 37(3), pp. 270-278.

Beysens, D.A., Forgacs, G. \& Glazier, J.A., 2000. Cell sorting is analogous to phase ordering in fluids. Proceedings of the National Academy of Sciences, 97(17), pp.9467-9471.

Borenstein, J. et al., 2002. Microfabrication Technology for Vascularized Tissue Engineering. Biomedical Microdevices, pp.167175.

Damon, B.J. et al., 2008. Limb bud and flank mesoderm have distinct "physical phenotypes" that may contribute to limb budding. Developmental Biology, 321(2), pp.319-330.

Duguay, D., Foty, R.A. \& Steinberg, M.S., 2003. Cadherin-mediated cell adhesion and tissue segregation: qualitative and quantitative determinants. Developmental Biology, 253(2), pp.309-323. 
Eshelby, J.D., 1949. Trans. Am. Inst. Min., Metall. Pet. Eng., 185, pp. 806-807.

Evans, E. \& Yeung, A., 1989. Apparent viscosity and cortical tension of blood granulocytes determined by micropipet aspiration. Biophysj, 56(1), pp.151-160.

Forgacs, G. \& Newman, S.A., 2005. Biological physics of the developing embryo.

Forgacs, G. et al., 1998. Viscoelastic properties of living embryonic tissues: a quantitative study. Biophysj, 74(5), pp.2227-2234.

Foty, R.A. \& Steinberg, M.S., 2005. The differential adhesion hypothesis: a direct evaluation. Developmental Biology, 278(1), pp.255-263.

Foty, R.A. et al., 1994. Liquid properties of embryonic tissues: measurement of interfacial tensions. Physical Review Letters, 72(14), pp.2298-2301.

Foty, R.A. et al., 1996. Surface tensions of embryonic tissues predict their mutual envelopment behavior. Development, 122(5), pp.1611-1620.

Frenkel, J., 1945. Viscoud Flow of Crystalline Bodies Under the Action of Surface Tension. Journal of Physics, 9(5), pp.385-391.

Gauvin, R. et al., 2012. Microfabrication of complex porous tissue engineering scaffolds using 3D projection stereolithography. Biomaterials, 33(15), pp.3824-3834.

Greenwood, H.L. et al., 2006. Regenerative medicine: new opportunities for developing countries. International journal of biotechnology, 8(1), pp.60-77.

Griffith, L.G. \& Naughton, G., 2002. Tissue engineering--current challenges and expanding opportunities. science, 295(5557), pp.1009-1014.

Guevorkian, K. et al., 2010. Aspiration of Biological Viscoelastic Drops. Physical Review Letters, 104(21), pp.218101-218104.

Hegedüs, B. et al., 2006. The Interplay of Cell-Cell and Cell-Matrix Interactions in the Invasive Properties of Brain Tumors. Biophysj, 
91(7), pp.2708-2716.

Ingber, D.E. et al., 2006. Tissue engineering and developmental biology: going biomimetic. Tissue engineering, 12(12), pp.32653283.

Jakab, K. et al., 2004. Engineering biological structures of prescribed shape using self-assembling multicellular systems. Proceedings of the National Academy of Sciences, 101(9), pp.2864-2869.

Jakab, K. et al., 2010. Tissue engineering by self-assembly and bioprinting of living cells. Biofabrication, 2(2), p.022001.

Jakab, K. et al., 2008. Tissue Engineering by Self-Assembly of Cells Printed into Topologically Defined Structures. Tissue Engineering Part A, 14(3), pp.413-421.

Kalantarian, A. et al., 2009. Axisymmetric Drop Shape Analysis for Estimating the Surface Tension of Cell Aggregates by Centrifugation. Biophysj, 96(4), pp.1606-1616.

Khademhosseini, A. et al., 2006. Microscale technologies for tissue engineering and biology. Proceedings of the National Academy of Sciences, 103(8), pp.2480-2487.

Khademhosseini, A., Vacanti, J.P. \& Langer, R., 2009. Progress in tissue engineering. Scientific American, 300(5), pp.64-71.

Kosztin, I., Vunjak-Novakovic, G. \& Forgacs, G., 2012. Colloquium: Modeling the dynamics of multicellular systems: Application to tissue engineering. Reviews of Modern Physics, 84(4), pp.17911805.

Langer, R., 2007. Editorial: Tissue Engineering: Perspectives, Challenges, and Future Directions. Tissue engineering, 13(1), pp.12.

Langer, R., 2009. Perspectives and Challenges in Tissue Engineering and Regenerative Medicine. Advanced Materials, 21(32-33), pp.3235-3236.

Langer, R. \& Vacanti, J., 1993. Tissue Engineering. science, 260(5110), pp.920-926.

Lanza, R., Langer, R. \& Vacanti, J.P., 2013. Principles of Tissue 
Engineering, Academic Press.

Lecuit, T. \& Lenne, P.-F., 2007. Cell surface mechanics and the control of cell shape, tissue patterns and morphogenesis. Nature Reviews Molecular Cell Biology, 8(8), pp.633-644.

Lenas, P., Moos, M., Jr \& Luyten, F.P., 2009. Developmental Engineering: A New Paradigm for the Design and Manufacturing of Cell-Based Products. Part I: From Three-Dimensional Cell Growth to Biomimetics of In VivoDevelopment. Tissue Engineering Part B: Reviews, 15(4), pp.381-394.

Lu, H.H. et al., 2003. Three-dimensional, bioactive, biodegradable, polymer-bioactive glass composite scaffolds with improved mechanical properties support collagen synthesis and mineralization of human osteoblast-like cells in vitro. Journal of Biomedical Materials Research Part A, 64(3), pp.465-474.

Macchiarini, P. et al., 2008. Clinical transplantation of a tissueengineered airway. The Lancet, 372(9655), pp.2023-2030.

Manning, M.L. et al., 2010. Coaction of intercellular adhesion and cortical tension specifies tissue surface tension. Proceedings of the National Academy of Sciences, 107(28), pp.12517-12522.

Marga, F. et al., 2008. Developmental biology and tissue engineering. Birth Defects Research Part C: Embryo Today: Reviews, 81(4), pp.320-328.

Marmottant, P. et al., 2009. The role of fluctuations and stress on the effective viscosity of cell aggregates. Proceedings of the National Academy of Sciences, 106(41), pp.17271-17275.

Mason, C. \& Dunnill, P., 2008. A brief definition of regenerative medicine. Regenerative Medicine, 3(1), pp.1-5.

McCune, M. et al., 2014. Predictive modeling of post bioprinting structure formation. Soft Matter, 10(11), pp.1790-1800.

Merrill, J.P. et al., 1956. Successful homotransplantation of the kidney in an identical twin. Transactions of the American Clinical and Climatological Association, 67, pp.166-173.

Mgharbel, A., Delanoë-Ayari, H. \& Rieu, J.-P., 2009. Measuring 
accurately liquid and tissue surface tension with a compression plate tensiometer. HFSP Journal, 3(3), pp.213-221.

Mombach, J.C. et al., 1995. Quantitative comparison between differential adhesion models and cell sorting in the presence and absence of fluctuations. Physical Review Letters, 75(11), pp.22442247.

Murray, J.E. et al., 1968. Five years' experience in renal transplantation with immunosuppressive drugs: survival, function, complications, and the role of lymphocyte depletion by thoracic duct fistula. Annals of surgery, 168(3), pp.416-433.

Murray, J.E. et al., 2001. Renal homotransplantation in identical twins. J Am Soc Nephrol, 12(1), pp.201-204.

Norotte, C., 2009. From Developmental Biology to Tissue-Engineering Printing Blood Vessels. Columbia: University of Missouri.

Norotte, C. et al., 2008. Experimental evaluation of apparent tissue surface tension based on the exact solution of the Laplace equation. EPL (Europhysics Letters), 81(4), p.46003.

Norotte, C. et al., 2009. Scaffold-free vascular tissue engineering using bioprinting. Biomaterials, 30(30), pp.5910-5917.

Oberpenning, F. et al., 1999. De novo reconstitution of a functional mammalian urinary bladder by tissue engineering. Nature biotechnology, 17(2), pp.149-155.

Persidis, A., 1999. Tissue Engineerng. Nature biotechnology, 17, pp.508-510.

Pérez-Pomares, J.M. \& Foty, R.A., 2006. Tissue fusion and cell sorting in embryonic development and disease: biomedical implications. BioEssays, 28(8), pp.809-821.

Raya-Rivera, A. et al., 2011. Tissue-engineered autologous urethras for patients who need reconstruction: an observational study. The Lancet, 377(9772), pp.1175-1182.

Raya-Rivera, A.M. et al., 2014. Tissue-engineered autologous vaginal organs in patients: a pilot cohort study. The Lancet.

Sala, C.C. et al., 2013. Current Applications of Tissue Engineering in 
Biomedicine. J Biochip Tissue chip S, 2, pp.2153-0777.

Sato, M. et al., 1990. Application of the micropipette technique to the measurement of cultured porcine aortic endothelial cell viscoelastic properties. Journal of Biomechanical Engineering, 112(3), pp.263268.

Schötz, E.M. et al., 2008. Quantitative differences in tissue surface tension influence zebrafish germ layer positioning. HFSP Journal, 2(1), pp.42-56.

Starzl, T.E., 1984. The landmark identical twin case. JAMA, 251(19), pp.2572-2573.

Steinberg, M.S., 1998. Goal-Directedness in Embryonic Development. Integrative Biology, 1, pp.49-59.

Steinberg, M.S., 1963. Reconstruction of tissues by dissociated cells. science, 141(3579), pp.401-408.

Steinberg, M.S., 1978. Specific cell ligands and the differential adhesion hypothesis: how do they fit together?, pp.97-130.

Vacanti, J., 2010. Tissue engineering and regenerative medicine: from first principles to state of the art. Journal of Pediatric Surgery, 45(2), pp.291-294.

Yoo, J.J. et al., 1998. Bladder augmentation using allogenic bladder submucosa seeded with cells. Urology, 51(2), pp.221-225. 


\section{Appendix A: Finding the ATST using "Exact Solution of Laplace equation"}

Here we briefly summarize formulas from Norotte, C. et al., 2008, to find the exact solution of Laplace equation and finding the ATST consequently.

These authors determined $R_{1}, R_{2}$ and $R_{3}$ by finding the exact profile $z(r)$ of a compressed aggregate using the exact solution of the Laplace equation.

$\Delta p=\gamma\left[\frac{z^{\prime}}{r\left(1+z^{\prime 2}\right)^{1 / 2}}+\frac{z^{\prime \prime}}{\left(1+z^{\prime 2}\right)^{3 / 2}}\right]=$ const

Here $z^{\prime}$ and $z^{\prime \prime}$ are, respectively, the first and second derivative of $z(r)$ with respect to $r$. The above equation needs to be solved with the following boundary conditions (see Fig. 6):

$z\left(R_{3}\right)=0, z^{\prime}\left(R_{3}\right)=\tan \theta$

$z\left(R_{1}\right)=H / 2, z^{\prime}\left(R_{1}\right)=\infty$

The solution of the Laplace equation then yielded $\gamma$ in terms of $H, R_{1}$ and $F$ that can be found accurately experimentally (see Fig. 6). Below we summarize the mathematical details.

$R_{2}=R_{1} /(2 \alpha-1)$ 
$R_{3}=\beta_{\theta} R_{1}$

where $\alpha$ and $\beta_{\theta}$ are dimensionless parameters defined by:

$\alpha=\Delta p /\left(2 \gamma / R_{1}\right)$

$\beta_{\theta} \equiv \beta_{\theta}(\alpha)=\frac{\sin \theta+\sqrt{\sin ^{2} \theta+4 \alpha(\alpha-1)}}{2 \alpha}$

Integration of equation (A.1) with its boundary conditions A.2 and A.3 helps to find an implicit equation for $\alpha$.

$\frac{H}{2 R_{1}}=f_{\theta}(\alpha) \equiv \int_{\beta_{\theta}}^{1} z^{\prime}(x) d x$

$z^{\prime}(x)=\left[\left(\frac{x}{\alpha x^{2}+1-\alpha}\right)^{2}-1\right]^{\frac{-1}{2}}$

and the lateral profile of the compressed aggregate

$z(r)=R_{1} \int_{\beta_{\theta}}^{r / R_{1}} z^{\prime}(x) d x$

For small contact angles, $\alpha=f_{\theta}^{-1}\left(H / 2 R_{1}\right)$. Finally, $\gamma$ can be found from

$F / 2 \pi R_{1}=\gamma(\alpha-1)$.

The quantity $\alpha$ is obtained from numerically performing the integration in (A.12). The surface tension $\gamma$ is obtained by plotting $F / 2 \pi R_{1}$ versus $(\alpha-1)$ and determining the slope of this plot. 


\section{Appendix B: Theory background for fusion of spherical aggregates}

Here we summarize the essential formulas, from McCune et.al. 2013, that we needed to analyze the fusion of two spherical aggregates.

One can define $\frac{R(\theta)}{R_{0}}$ as:

$\rho(\theta) \equiv \frac{R(\theta)}{R_{0}}=2^{2 / 3}(1+\cos \theta)^{-2 / 3}(2-\cos \theta)^{-1 / 3}$

$\mathrm{R}_{0}$ is the radius of the aggregate for $\theta=0$. With this the fusion process can be described in terms of the fusion angle $\theta=\theta(t)$, which varies from $\theta(0)=0$ (when the fusion starts) to $\theta(\infty)=\pi / 2$ (when the fusion ends). Fusion terminates when the two spherical aggregates completely merge into one..

Ii is convenient to introduce the characteristic fusion time $\tau$ as

$\tau=\eta R_{0} / \gamma$

The dynamics of the fusion process, described by $\theta(t)$ as a function of $t / \tau$ is independent of size (i.e., $R_{0}$ ), $R_{0}$ only appears in fusion time Eq. (B.2). Furthermore, the square of the radius of the circular neck region of the fusing spherical caps can be expressed as

$\left(\frac{r}{R}\right)^{2}=\sin ^{2} \theta \approx 1-\exp (-t / \tau)$ 
Note that for $t \ll \tau$, Eq. (B.3) reduces to the linear in-time expression $\left(\frac{r}{R}\right)^{2} \approx t / \tau$ obtained by Frankel (1945) and Eshleby (1949).

To describe the case non-conserved aggregate volume we assume that the decrease in the radius of aggregate is governed by $R_{0}(t)=a(t) R_{0}(0)$, where $\mathrm{a}(\mathrm{t})$ is a time dependent dimensionless quantity. In the experiments we have performed and reported in this thesis $\mathrm{a}(\mathrm{t})$ can be approximated by the expression

$a(t)=a_{0}+\left(1-a_{o}\right) \exp (-\lambda t)$

where $a_{0}=a(\infty) \leq 1$ is the relative change of the linear dimension of the system during its complete volume relaxation, and $\lambda$ is the rate of this process.

The theory can be extended from same size aggregates to the different size cell aggregates. Here again one can first consider constant aggregate volume and then by generalize the theory to the case of non-conserved.

The fusion of different size (uneven) aggregates is modeled as two spherical caps of radii $R_{1}\left(\theta_{1}\right)>R_{2}\left(\theta_{2}\right)$ with a circular contact "neck" region of radius

$r=R_{1} \sin \theta_{1}=R_{2} \sin \theta_{2}$. 
The fusion angles are defined in Fig.11. The initial radii of the uneven aggregates are denoted as $R_{i 0}=R_{i}(0), i=1,2$. Without the loss of generality we set $R_{1}\left(\theta_{1}\right)>R_{2}\left(\theta_{2}\right)$ and intoduce $b=\frac{R_{10}}{R_{20}}>1$.

For the constant volume case we can extend Eq. (B.1) to

$\rho_{i} \equiv \rho\left(\theta_{i}\right) \equiv \frac{R_{i}\left(\theta_{i}\right)}{R_{i 0}}=2^{2 / 3}\left(1+\cos \theta_{i}\right)^{-2 / 3}\left(2-\cos \theta_{i}\right)^{-1 / 3}$.

Hence again the dynamics of the fusion process is completely determined by the time dependence of the fusion angles $\theta_{1}(t)$ and $\theta_{2}(t)$ which are related through Eq. (B.5). $\theta_{1}(t)$ and $\theta_{2}(t)$ change from $\theta_{1}(0)=\theta_{2}(0)=0$ to $\theta_{1}(\infty)=\theta_{\max }$ and $\theta_{2}(\infty)=\pi-\theta_{\max }$. Again, the fusion time is

$\tau=\eta R_{10} / \gamma$

Similarly to the same size aggregates, the fusion angle $\theta_{1}$ (and, implicitly, $\theta_{2}$ ) is only a function of $\frac{t}{\tau}$ and does not depend explicitly on the size of the system although it depends on $b=\frac{R_{10}}{R_{20}}$. Then the only radius that appears in the Eq. (B.7) is $R_{10}$. The case of non-conserved volume can be described similarly as it was done for the same size aggregates above. 


\section{VITA}

Ashkan Shafiee was born in Tehran, Iran. He received his BSc in Physics from Bu-Ali-Sina University in Hamedan, Iran in 2006. To obtain a Master's degree in Microengineering and Nanoelectronics he joined the organic electronic laboratory at the Institute of Microengineering and Nanoelectronics (IMEN) at the National University of Malaysia. For his master's project he worked with printers and used them in electronic applications. After working in printed electronics for almost 4 years, to extend his knowledge in printing for biomedical applications, he joined Dr. Gabor Forgacs laboratory in 2010 at University of Missouri and received his second MSc and a PhD in Biological Physics. He accepted a postdoctoral research fellow position at Wake Forest Institute for Regenerative Medicine and Wake Forest School of Medicine in North Carolina, under supervision of Dr. Anthony Atala. 